\title{
WestVirginiaUniversity
}

THE RESEARCH REPOSITORY @ WVU

Graduate Theses, Dissertations, and Problem Reports

2001

\section{Software tool for reliability estimation}

Manish Jhunjhunwala

West Virginia University

Follow this and additional works at: https://researchrepository.wvu.edu/etd

\section{Recommended Citation}

Jhunjhunwala, Manish, "Software tool for reliability estimation" (2001). Graduate Theses, Dissertations, and Problem Reports. 1146.

https://researchrepository.wvu.edu/etd/1146

This Thesis is protected by copyright and/or related rights. It has been brought to you by the The Research Repository @ WVU with permission from the rights-holder(s). You are free to use this Thesis in any way that is permitted by the copyright and related rights legislation that applies to your use. For other uses you must obtain permission from the rights-holder(s) directly, unless additional rights are indicated by a Creative Commons license in the record and/ or on the work itself. This Thesis has been accepted for inclusion in WVU Graduate Theses, Dissertations, and Problem Reports collection by an authorized administrator of The Research Repository @ WVU. For more information, please contact researchrepository@mail.wvu.edu. 


\title{
Software Tool for Reliability Estimation
}

\author{
Manish Jhunjhunwala
}

Thesis submitted to the College of Engineering and Mineral Resources at West Virginia University in partial fulfillment of the requirements for the degree of

\author{
Master of Science \\ in \\ Industrial Engineering
}

Rashpal S. Ahluwalia, Chair

Majid Jaraiedi

Department of Industrial and Management Systems Engineering,

Kenneth McGill

NASA Software IV \& V Facility, Fairmont, West Virginia.

Morgantown, West Virginia

2001

Keywords: Hardware Reliability, Software Reliability, System Reliability, Reliability Tool 


\section{ABSTRACT \\ Software Tool for Reliability Estimation}

\section{Manish Jhunjhunwala}

Reliability engineering is now an essential part of product development. In the area of electronics, reliability needs to be checked for each critical component before the product is shipped. Electronic products can range from computers to pace makers.

Over the years, several tools have been developed to estimate hardware/software reliability. Typically, such tools are either for hardware or software. This thesis presents a Software Tool for Reliability Estimation (STORE), which can be used for hardware components, software components, and for systems having both hardware and software components.

The objective of this research was to study several hardware-software reliability models, existing algorithms to compute system reliability and to model state dependent systems. A comprehensive software tool to estimate reliability of hardware and software component that are state dependent and independent was developed. This software tool was implemented in Visual Basic for a Windows 98 operating system. 


\section{Acknowledgment}

I would like to express my sincere thanks and gratitude to Dr. Rashpal Ahluwalia, my committee chair for his continuous support throughout this research. He was always available to answer my questions and explicate problems that I encountered. I also appreciate the time and effort of Dr. Majid Jaraiedi and Mr. Kenneth McGill for their helpful comments and suggestions.

I am thankful to the Industrial and Management Systems Engineering Department for its continued financial support which enabled me to complete my graduate work. Finally, I would like to thank my parents, my brother and all my friends for their support all through my studies. 


\section{Table of Contents}

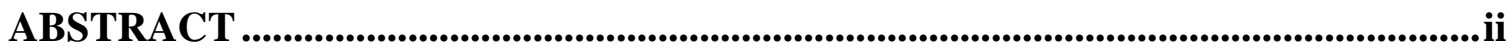

ACKNOWLEDGMENT ...............................................................................................................ii

TABLE OF CONTENTS........................................................................................................iv

LIST OF NOTATIONS ................................................................................................. vii

LIST OF FIGURES _..................................................................................................................ix

LIST OF TABLES …..............................................................................................................

CHAPTER 1: INTRODUCTION .............................................................................1

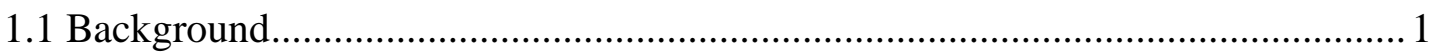

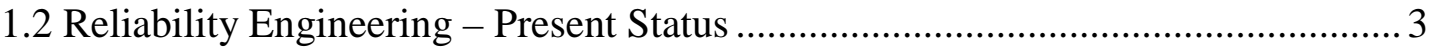

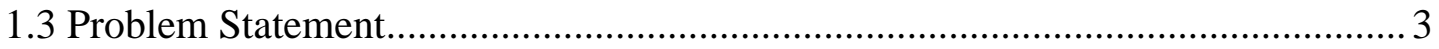

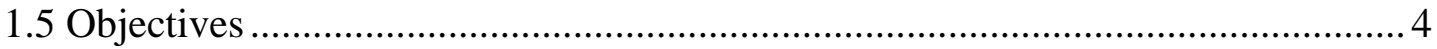

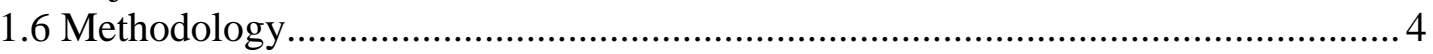

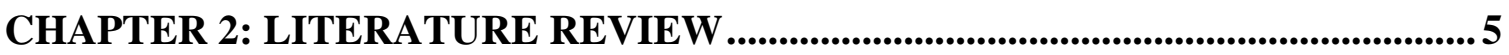

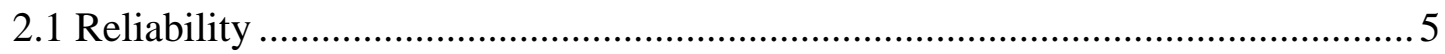

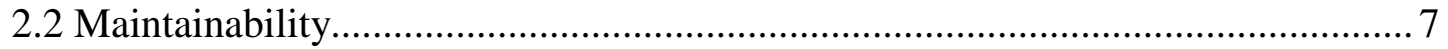

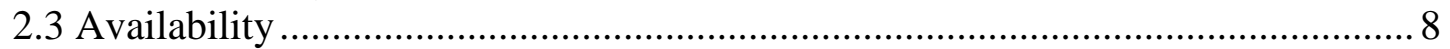

CHAPTER 3: HARDWARE RELIABILITY MODELS..........................................9

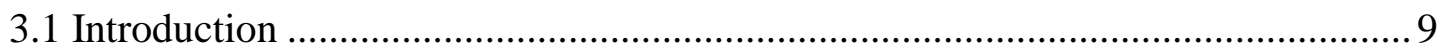

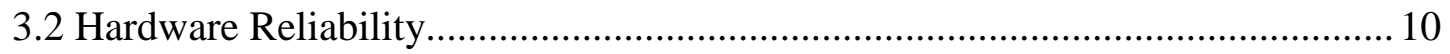

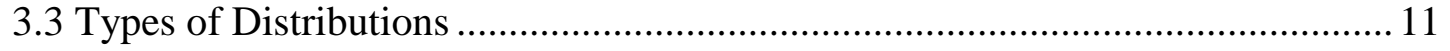

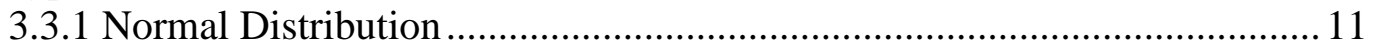

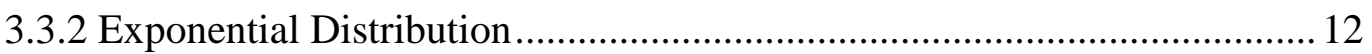

3.3.3 Weibull Distribution .............................................................................. 12

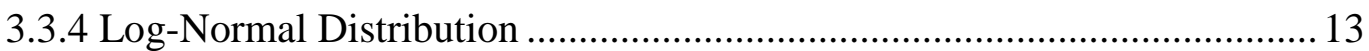

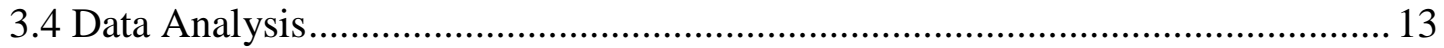

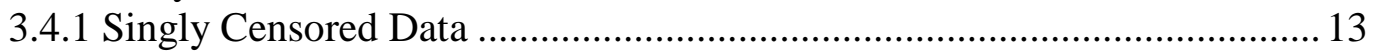

3.4.2 Least Square Estimates ........................................................................... 14

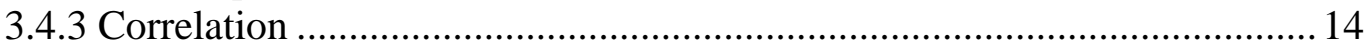

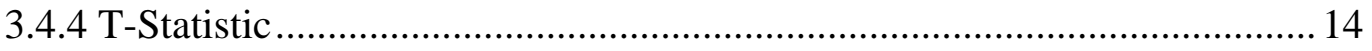

3.4.5 Maximum Likelihood Estimators (MLE) ……………………………..... 15

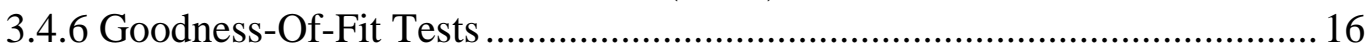

CHAPTER 4: SOFTWARE RELIABILITY ............................................................. 17

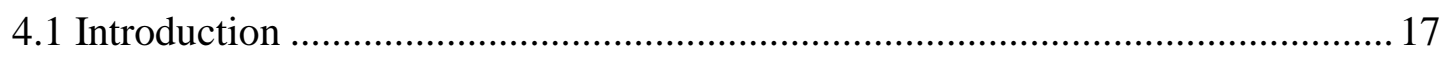

4.2 Software Reliability Estimation......................................................................2 20

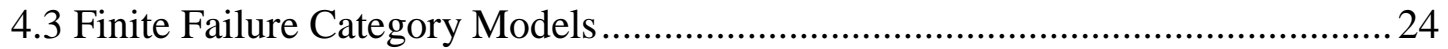

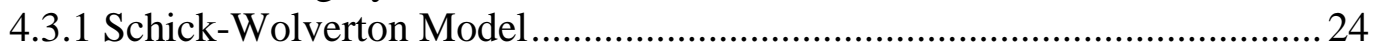

4.3.2 Basic Musa Model ………………………………........................... 25

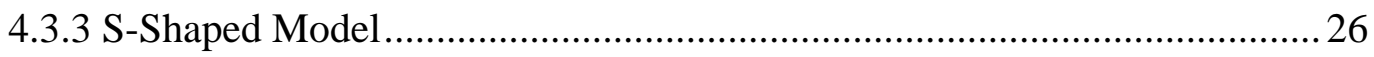

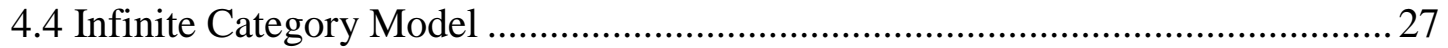


4.4.1 Duane's Model....................................................27

4.4.2 Logarithmic Poisson Model .................................................................. 28

4.4.3 Inverse Linear Model ................................................................................ 28

CHAPTER 5: SYSTEM RELIABILITY .............................................................29

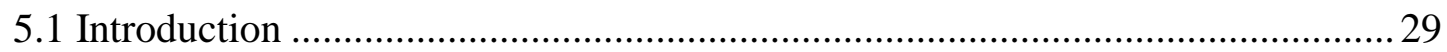

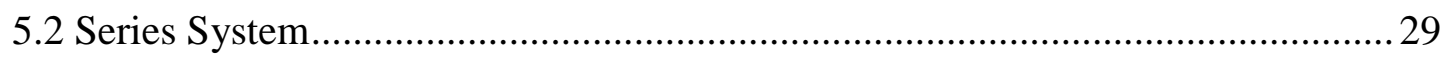

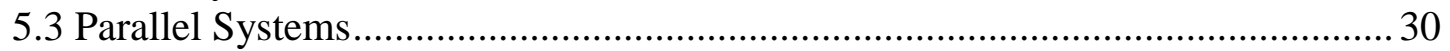

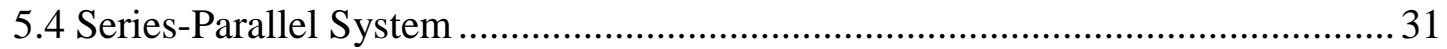

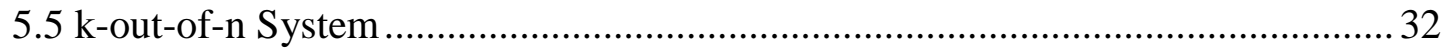

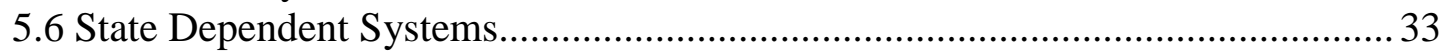

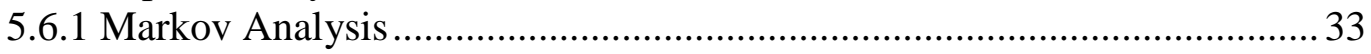

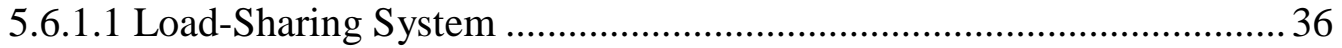

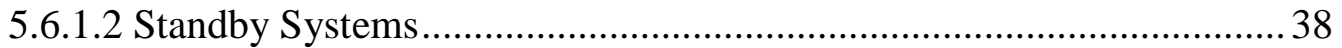

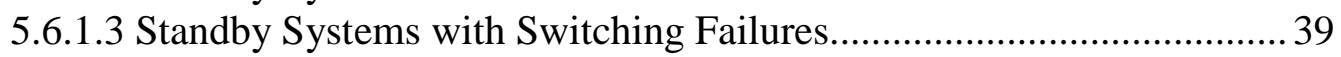

5.6.1.4 Three-Component Standby System .............................................. 40

5.6.1.5 Degraded Systems ........................................................................ 41

5.7 State Independent System Reliability - Tie Set and Cut Set Algorithm .............. 42

5.7.1 Tie Set or Minimal Paths Algorithm......................................................... 42

5.7.2 Determination of Cut sets from given Minimal Paths .............................. 43

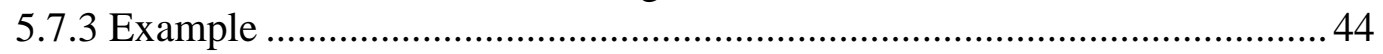

5.7.3 Algorithm for Calculating System Reliability ...................................... 45

\section{CHAPTER 6: SOFTWARE TOOL FOR RELIABILITY ESTIMATION (STORE)}

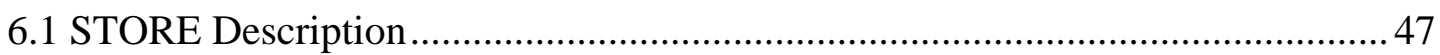

6.2 STORE Menu Structure ............................................................................ 48

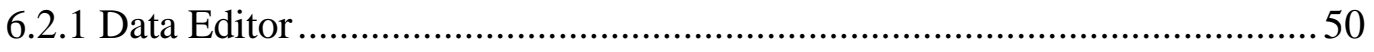

6.2.2 Hardware Reliability - Complete Data....................................................5 51

6.2.3 Hardware Reliability - Censored Data ................................................ 52

6.2.4 Software Reliability ........................................................................... 53

6.2.5 Result Screen ................................................................................. 53

6.2.6 System Reliability - State Dependent..................................................... 54

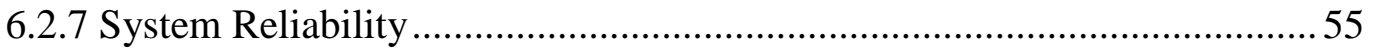

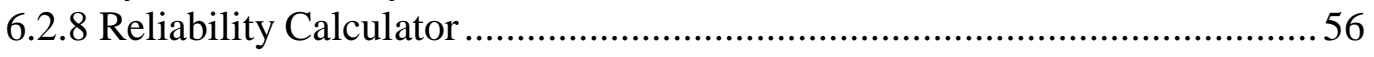

CHAPTER 7: STORE APPLICATION .....................................................................58

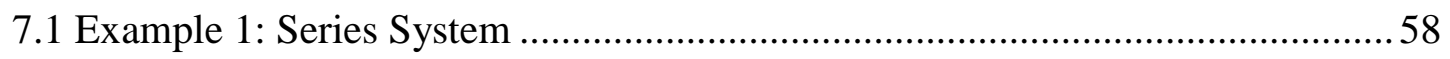

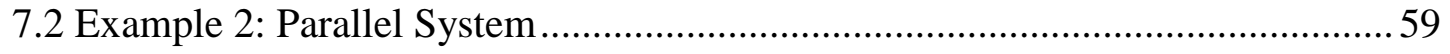

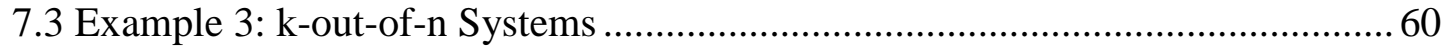

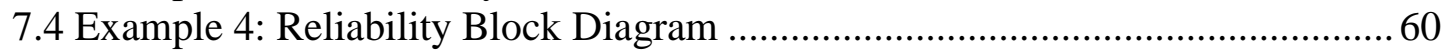

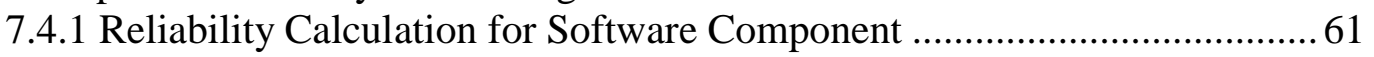

7.4.2 Calculation of Hardware Reliability - Complete Data ..............................6 63

7.1.3 Calculation of Hardware Reliability - Censored Data .............................6 65

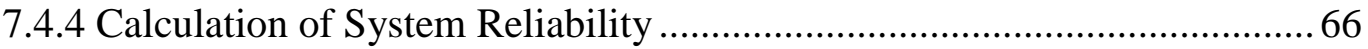

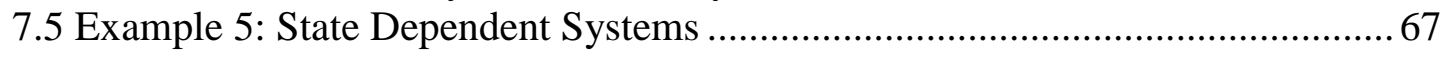

CHAPTER 8: CONCLUSIONS AND FUTURE WORK ........................................... 70

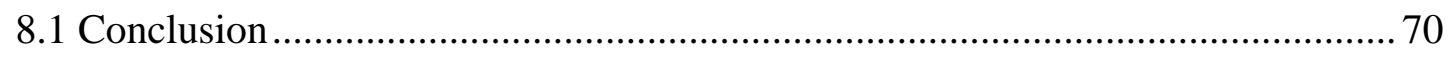

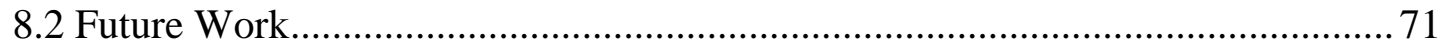


REFERENCES

APPENDIX I: BARTLETT'S TEST FOR EXPONENTIAL DISTRIBUTION ...... 75

APPENDIX II: KOLMOGOROV-SMIRNOV TEST .....................................................76

APPENDIX III: MANN'S TEST FOR WEIBULL DISTRIBUTION..........................77

APPENDIX IV: RELIABILITY BLOCK DIAGRAM INPUT ....................................78

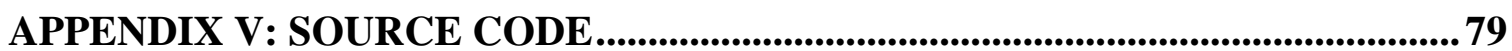


Bartlett's test statistic

$b_{0}, b_{1}, b_{2}$. $\quad$ Parameters for software models

$\mathrm{C}_{\mathrm{i}}$

$\mathrm{i}^{\text {th }}$ Cut set

cor

Correlation Coefficient

$\mathrm{E}[\mathrm{X}]$

Expected value of random variable $\mathrm{X}$

$\mathrm{F}(\mathrm{t})$

Probability Density Function

$\mathrm{i}, \mathrm{j}$

Indices indicating sequential number of failure events

$\mathrm{m}_{\mathrm{t}}$

Number of failures experienced by time $t$

m

Mean

$\mathrm{M}$

Mann's Test Statistic

$\mathrm{M}(\tau)$

Number of failures experienced by time $\tau$ (random variable)

$\mathrm{P}\left[\mathrm{E}_{1}\right]$

Probability of event $\mathrm{E}_{1}$

$\mathrm{P}\left[\mathrm{E}_{1} \mid \mathrm{E}_{2}\right.$

Probability of event $\mathrm{E}_{1}$ given event $\mathrm{E}_{2}$ occurred

$\mathrm{Q}_{\mathrm{s}}$

Unreliability of a system

$\mathrm{R}_{\mathrm{s}}$

Reliability of a system

$\mathrm{R}(\mathrm{t})$

Reliability function

$\sigma^{2}$

Variance

SSE

Sum of Squares of Errors

SSx

Sum of Squares of variable $\mathrm{X}$

SSy

Sum of Squares of variable Y

$\mathrm{t}_{\mathrm{e}}$

A specific deterministic time denoting the end of failure data.

$\mathrm{t}_{\mathrm{i}}$

Time of $i^{\text {th }}$ failure

$\mathrm{T}_{\text {med }}$

Shape parameter for lognormal distribution

$\mathrm{T}_{\mathrm{i}}$

$i^{\text {th }}$ Tieset

TS

T Statistic

MLE

Maximum Likelihood Estimator

$\mathrm{h}(\mathrm{t})$

Hazard rate

$\alpha$

Confidence level; significance of a model or probability of rejecting a correct model.

$\beta \quad$ Shape parameter for Weibull distribution (hardware reliability) 
$\lambda \quad$ Parameter for exponential distribution used in hardware reliability or the hazard rate

$\lambda(\tau) \quad$ Failure intensity function or $\mathrm{d} \mu / \mathrm{dt}$

$\mu \quad$ Mean

$\mu(\tau) \quad$ Mean value function or expected number of failures experienced by time $t$ or $\mathrm{E}[\mathrm{M}(\tau)]$

$\sigma \quad$ Standard deviation

$\tau \quad$ (Realization of) cumulative execution time

$\gamma \quad$ Location parameter for Weibull distribution

$\eta \quad$ Scale parameter for Weibull distribution 


\section{List of Figures}

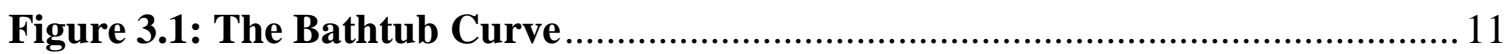

Figure 4.1: Software Reliability Measurement Procedure Overview …………....... 21

Figure 4.2: Failure Intensity Curve and Mean Value Function for a Non-

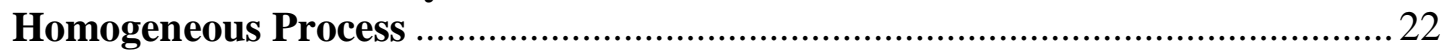

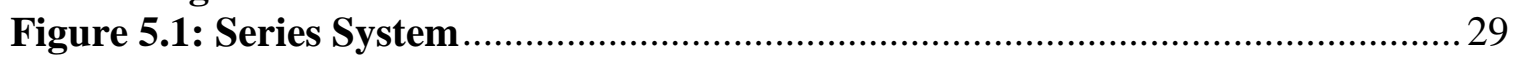

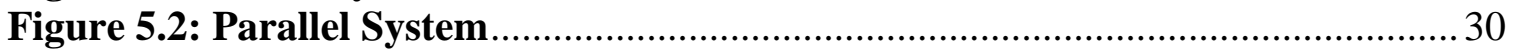

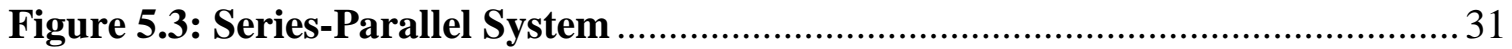

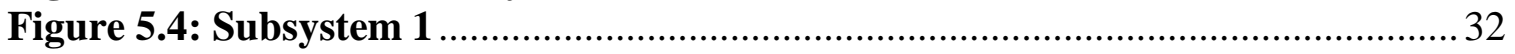

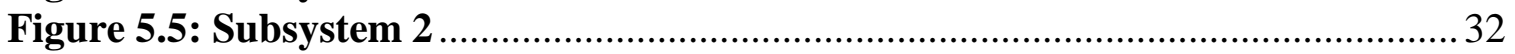

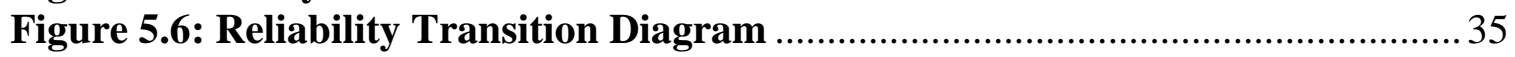

Figure 5.7: Reliability Transition Diagram for Load Sharing System....................... 37

Figure 5.8: Reliability Transition Diagram for Standby System .............................. 38

Figure 5.9: Reliability Transition Diagram for Standby Systems with Switching

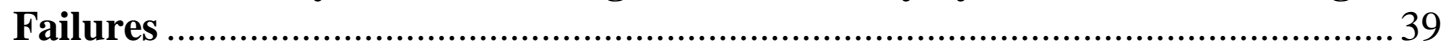

Figure 5.10: Reliability Transition Diagram for Degraded Systems ......................... 41

Figure 5.11: Complex System............................................................................. 44

Figure 5.12: Tree Structure of the Complex System................................................ 44

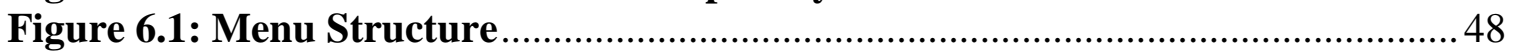

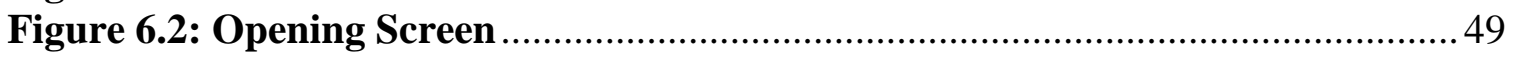

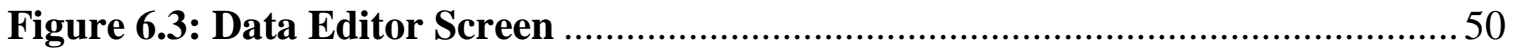

Figure 6.4: Hardware Reliability - Complete data screen ……………………….... 51

Figure 6.5: Hardware Reliability - Censored data screen ……….............................5 52

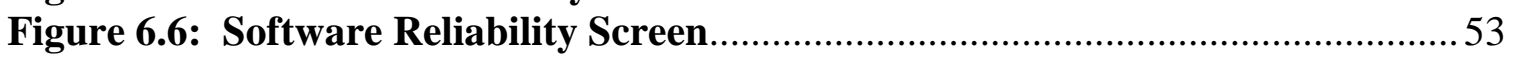

Figure 6.7: Result Screen for Software Reliability ..............................................5 54

Figure 6.8: State Dependent System Reliability Screen...........................................5

Figure 6.9: System Reliability Screen ..............................................................5 56

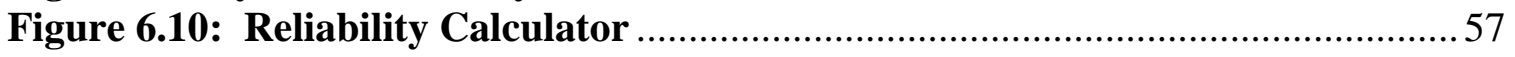

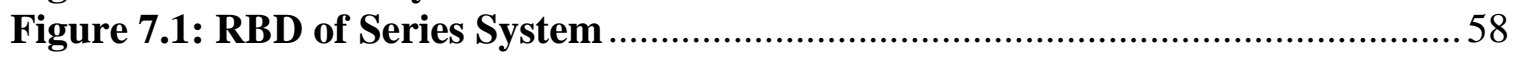

Figure 7.2: Sample STORE Screen for System Reliability of Series System.............58

Figure 7.3: RBD for Parallel System .................................................................5

Figure 7.4: Sample STORE Screen for System Reliability of Parallel System ……. 59

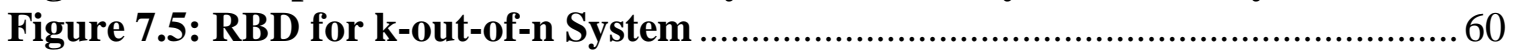

Figure 7.6: Sample STORE Screen for System Reliability of k-out-of-n System ..... 60

Figure 7.7: Reliability Block Diagram for a Hardware - Software System ...............61 61

Figure 7.8: Sample STORE Screen for the Exponential Model (Software) ...............63

Figure 7.9: Sample STORE Screen for the Exponential Model (Hardware) ............ 65

Figure 7.10: Hardware Reliability - Censored Data Screen ......................................66

Figure 7.11: System Reliability Screen .................................................................. 67

Figure 7.12: Sample STORE Screen Showing State Dependent System Reliability

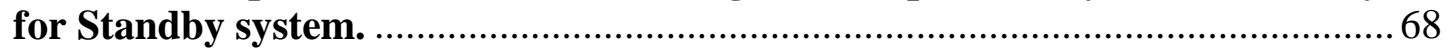




\section{List of Tables}

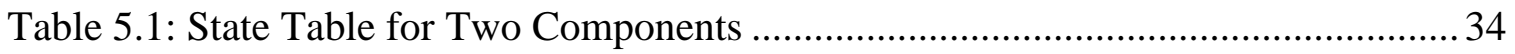

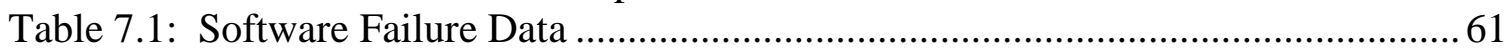

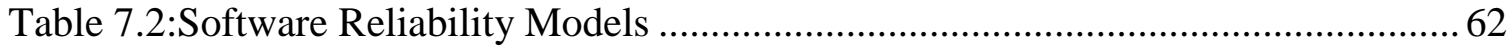

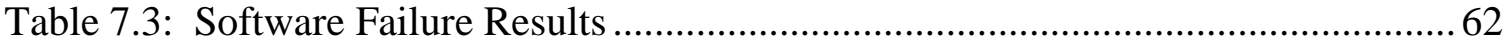

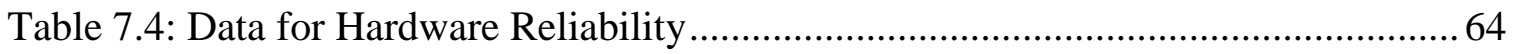

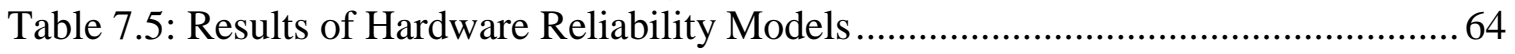

Table 7.5: Data for Hardware Reliability - Censored Data .......................................6. 65

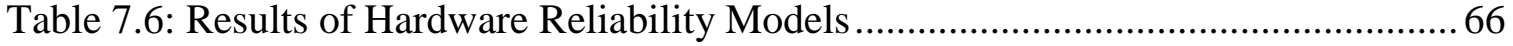

Table 7.7: Reliability and MTTF for State Dependent Systems .................................... 69 


\section{Chapter 1: Introduction}

\subsection{Background}

Since the first electronic digital computer was invented almost fifty years ago, we have become dependent on computers in our daily lives. The computer revolution has created the fastest technological advancement that the world has ever seen. Today, the computer hardware and software applications are pervasive in all aspects of our society. The newest cameras, VCRs, and automobile could not be controlled and operated without computers. Computers are also embedded in wristwatches, telephones, home appliances, buildings, and aircraft. Science and technology have been demanding high performance hardware and high quality software for making improvements and breakthroughs. We can look at virtually any industry, automotive, avionics, oil, telecommunications, banking, semiconductor, pharmaceutics, etc., and see that they rely heavily on computers for their functioning [24].

The size and complexity of computer intensive systems have grown dramatically, and this trend will certainly continue in the future. Contemporary examples of highly complex hardware/software systems can be found in projects undertaken by NASA, Department of Defense (DoD), the Federal Aviation Administration (FAA), the telecommunications industry and a variety of other private industries.

The demand for complex hardware/software systems has increased more rapidly than the ability to design, implement, test and maintain them. When dependency on computers increases, the possibility of crises from computer failures also increases. The impact of these failures ranges from inconvenience (e.g., malfunctions of home appliances), economic damage (e.g., interruptions of banking systems), to loss of life (e.g., failures of flight systems or medical software).

As the system becomes more complex, data collection becomes tougher. That is, most of the experience with the models is during the testing phase of actual projects, during which researchers have little or no control over the data with which they work. The complexity of real world failure data may obscure properties of software reliability models that might be revealed by executing the models on a simpler data sets [22].

As per Nikora [22] most appropriate software reliability model is a process that continues throughout the testing phase. Even if the characteristics of the testing process 
are well-known for a particular development effort and whose assumptions best match these characteristics, there is no guarantee that the model will be most appropriate model. That is, reliance on a single measure to choose the most appropriate model can lead to making an incorrect choice.

The word reliability is used in everyday life in more qualitative terms than quantitative terms. It is often said that a particular equipment, motor, or automobile is reliable or unreliable, implying that it is dependable or not dependable, respectively. If a component is dependable, the possibility that it can ever fail cannot be ruled out.

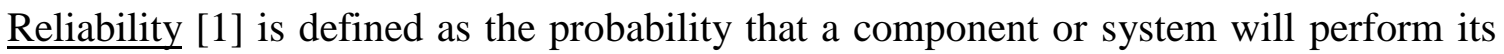
intended function for a given time period, when used under stated operating conditions. It is the probability of non-failure over time. According to the definition, it takes failure data to measure reliability. The failure data can be fitted to either constant failure rate distribution, such as exponential or time dependent failure distributions like Normal, Lognormal or Weibull distribution.

Maintainability [1] is defined as the probability that a failed component or a system will be restored or repaired to a specified condition within a period of time when maintenance is performed in accordance with prescribed procedures. Maintainability, is the probability of repair in a given time. Usually, when maintainability is computed, time is defined as the clock time. It may or may not include such measures as waiting time for maintenance personnel and parts, travel time, and administrative time. Often, maintainability refers to the inherent repair time, which includes only the hands-on repair of the failed unit and not any administrative or resource delay times.

Availability [1] is defined as the probability that a component or system is performing its required function at a given point in time when used under stated operating conditions. Availability may also be interpreted as percent time a component or a system is operating. It differs from reliability in that availability is the probability that the component is currently in a non-failure state even though it may have previously failed and been restored to its normal operating conditions. Therefore system availability can never be less than system reliability. Availability can be the preferred measure when a system or component can be restored since it accounts for both reliability and maintainability. 


\subsection{Reliability Engineering - Present Status}

Over the period of time, several techniques have been developed to systematically model and analyze real systems. These techniques are constantly being enhanced to improve the modeling process and to reduce the time required for analysis of that model. A well-modeled system may be too complex to be analyzed within a reasonable amount of time. On the other hand, an oversimplified model may not be able to depict the characteristics of a real system. A global solution to these problems is to reach a compromise between the model complexity and the model execution and analysis time.

Recently, a study was undertaken at the Jet Propulsion Laboratory (JPL) to determine the applicability of current software reliability measurement techniques, to JPL's development efforts. One of the findings was that there is no known method for identifying the model most applicable to a development effort prior to test. This can be mitigated, however by executing several models at once and using statistical methods to identify the model that is most likely to produce accurate results. From the study it was also found that more accurate predictions can be produced by combining the results of several models in a linear fashion [23].

\subsection{Problem Statement}

Reliability is an important parameter of any system. Various techniques are used to compute system reliability. These techniques can be classified into two categories. The first category consists of techniques that find an approximate reliability solution, whereas the second category consists of techniques that find the exact solution. Some of the commonly used techniques that find the exact network reliability are the conditional probability technique, the cut set technique and the tie set technique. For a large complex system the computations times for such techniques can be extensive. The approximate reliability solutions that make use of the simplifying assumptions are much faster than the exact solutions. The penalty paid for this fast computation is a certain degree of inaccuracy in the solution. Another limitation of the approximate solution is that it does not produce the symbolic expression for terminal reliability. Determining symbolic expression for reliability is important to reevaluate the system reliability if the reliability of an individual link has changed, or to improve the system reliability under a given cost constraint. 
Parekh[24] implemented various hardware and software models in Visual Basic. A major limitation of his system is that it does not compute system tiesets and cutsets and does not model state dependent systems. The focus of this work is to implement an algorithm to compute system tiesets and cutsets and to develop a procedure for analyzing state dependent systems.

\subsection{Objectives}

The objective of this research were as follows:

- Study existing statistical models to estimate reliability of Hardware-Software components.

- Study existing algorithm to compute reliability of complex systems.

- Implement an algorithm to compute tiesets and cutsets of complex systems.

- Compute reliability (with known or unknown parameters) of state dependent and state independent systems.

\subsection{Methodology}

To achieve the objectives of this research, the following methodology was carried out:

- Literature survey was done in the area of reliability and reliability estimation tool. Existing reliability tool as in [24] was enhanced to compute reliability of a system having software/hardware or both software and hardware components.

- The work was then documented explaining STORE and its application. Various examples were run on STORE and documented.

- STORE was made in Microsoft Visual Basic 6 to make it a user-friendly tool. This tool was integrated with hardware, software and system reliability. 


\section{Chapter 2: Literature Review}

This chapter reviews development of both hardware reliability and software reliability models, the purpose is to indicate which concepts have been tried successfully or not successfully and which have been modified and adapted. There are several distinct themes in historical development of this subject.

- The creation of various models relating reliability to failures times experienced.

- A concern with how to estimate model parameters.

- An interest in comparison of models, which led to the development of comparison criteria.

- The classification of models.

- An increasing concern with collecting failure data.

\subsection{Reliability}

Dimitri [4] describes the objective of reliability as follows:

1. Determine if the performance of components, equipment, and systems with or without corrective and preventive maintenance, and with known operating procedures, is within specifications for the desired function period, and if not, whether it is the result of a malfunction or of a failure which requires corrective action.

2. Determine the pattern of recurring failures, the cause of failures, and underlying times-to-failure distribution.

3. Determine the failure rate, the mean life, and the reliability of components, equipment, and systems and their associated confidence limits at the desired levels.

4. Based on the results obtained, provide guidelines as to whether corrective actions should be taken and what these should be.

5. Provide re-evaluation of the reliability-wise performance of the units after the corrective actions are taken to assure that these actions were the correct ones and as effective as intended.

6. Determine the growth in the mean life and/or the reliability of units during their 
research, engineering, and development phase, and whether the growth rate is sufficient enough to meet the mean life and/or the reliability need to be demonstrated.

7. Provide a means to statistically and scientifically determine, with chosen risks, whether a re-design has indeed improved the failure rate, mean life, or reliability of components or equipment with the desired confidence, and if not, what corrective actions need to be taken.

8. Provide a statistical and scientific means to determine which one of the two manufacturers of a component or equipment, or which design, should be preferred from the failure rate, mean life, or reliability point of view, all other factors being practically and economically the same, with the desired risk level.

Flehinger [10] has analyzed the reliability of complex systems in which components are used intermittently and which are maintained in operating condition by component replacement. The idea that a failed component causes system failure only when it is called into use is expressed mathematically. Based on component failure distributions and usage properties, the system reliability and expected time to system failure are derived as functions of system age for two different maintenance policies. With both policies, a component is replaced whenever it causes system failure. In the first case, this is the only maintenance, while in the second case, system checkout is conducted at fixed intervals and all components that have failed without causing system failure are replaced. The two policies are compared, and for the second, the dependence of system reliability on the maintenance interval in determined.

Raze [26] describes reliability prediction technique for mechanical equipment that gives a designer the ability to estimate the effects of varying design and operational constraints on a mechanical component's failure rate. The modeling process reduces the component down to its constituent parts and evaluates the effect of parametric changes on each part. This information is then used to develop both part and component failure rates. In addition, a validation-testing program was used to verify both the modeling technique and accuracy of the database information used. 


\subsection{Maintainability}

Goktas [11] developed an integrated reliability and maintainability model to study system design and operation problems. This model helps pick a cost efficient system design within appropriate system design and operating criteria. The following operating policies are used as availability improvement tools:

1. The use of backups at any stage in any number.

2. The use of buffers at any stage with any capacity.

3. The use of preventive maintenance at either component or the system level.

4. Corrective maintenance with any crew size.

5. Combination of the above listed choices.

Jardine [16] described quantitative procedures in the area of equipment maintenance. These procedures are useful in improving the performance of the maintenance function. The specific problem areas examined cover decisions relating to maintenance, replacement and reliability of industrial equipment. Additionally, decisions relating to the facilities required for maintenance, such as manpower requirements and equipment are also considered.

Boukas and Yang [3] present a solution to the problem of controlling the production rate and maintenance rate for a failure prone manufacturing system with one machine and one part so as to minimize the discounted inventory and maintenance cost. In the case where the cost is not dependent on maintenance activity, the optimal policy for maintenance is that the machine should be kept as good as new through maintenance. In the case where cost includes maintenance and if change of the machine age increases the cost significantly, maintenance is done such that the machine age is kept as constant. If the change of the machine age does not affect the cost significantly, no maintenance needs to be done. The optimal policy for production rate is given by a critical surface.

Ushakov [14] presents a series of optimal maintenance models. In the first model, there are repairs only after system failures; the failures are recognized immediately. For this model there is no optimization problem. In the second model, planned maintenance and repairs after system failures restores the system completely; the failures are recognized immediately. In the third model, only planned maintenance is performed. If a failure is found during the procedure, the system is repaired. In the fourth model, repair of a unit in a series system is performed after each failure. In the fifth model, repairs of a 
unit in a series system are performed after each failure, and the entire system has periodic planned maintenance.

Schouten and Vanneste [27] present optimal group maintenance policies for a set of identical machines subject to stochastic failures. The model presented can be of use to support the maintenance and replacement decisions for the system that are composed of a large number of identical components. In particular, this model focuses on the compromise between individual component replacements and complete system replacements. As such, it might be used to balance technological improvements of a system against the investment cost of such a system. The model contains a major decision variable $\mathrm{K}$, the number of doubtful components that triggers a system replacement. Two classes of policies are considered: A-policy replaces the whole system when a single component fails (or reaches its preventive maintenance age), while the number of doubtful components is greater than or equal to $\mathrm{K}$. The B-policy prescribes a system replacement at the first epoch at which an individual component fails after the first moment at which the number of doubtful components has reached level K. The validation of this model is performed by simulation. As a by-product, this validation reveals that simulation itself is of little use to support the decision process. It took very long simulation runs to obtain confidence intervals of an acceptable width for the average costs and the expected time between system replacements.

\subsection{Availability}

Dagpunar and Jack [5] established methods for assessing the availability of a system under minimal repair with constant repair times. A new policy and an existing policy for this type are discussed. Each involves replacement at the first failure after time $\mathrm{T}$, with $\mathrm{T}$ representing total operating time in the existing model and total elapsed time (i.e., operating time + repair time) in the new model. Optimal values of $\mathrm{T}$ are found for both policies over a wide range of parameter values. These results indicate that the new and the administratively easier policy produce only marginally smaller optimal availability values than the existing policy. 


\section{Chapter 3: Hardware Reliability Models}

\subsection{Introduction}

Reliability Engineering, as a separate engineering discipline, originated in the United States during the 1950s. The increasing complexity of military electronic systems was generating failure rates, which resulted in generally reduced availability and increased cost. Solid state electronics technology offered long-term hope, but conversely miniaturization was to lead to proportionately greater complexity, which offset the reliability improvements expected.

The rapid pace of electronic device technology meant that the developers of new military systems were making increasing use of large numbers of new component types, involving new manufacturing processes, with the inevitable consequences of low reliability. The users of such equipment were also finding that the problems of diagnosing and repairing the new complex system were seriously affecting its availability for use, and the cost of spares, training and other logistics support were becoming excessive.

Meanwhile the revolution in electronic device technology continued, led by integrated micro-circuitry. Increased emphasis was now placed on improving the quality of devices fitted to production equipment. Screening techniques, in which devices are temperature cycled, vibrated, centrifuged, operated at electrical overstress and otherwise abused, were introduced in place of the traditional sampling techniques. With component populations on even a single printed circuit board becoming so large, sampling no longer provided sufficient protection against the production of defective equipment.

The concept of life cycle costs (LCC), or whole life costs, was introduced. In the United Kingdom, Defense Standard 00-40, the Management of Reliability and Maintainability was issued in 1981. The British Standards Institution has issued BS 5760-Guide on Reliability of systems, equipment and components.

In this chapter a number of terms related to reliability, such as reliability function, expected life, hazard rate, will be defined and one important reliability function will be described. These concepts apply to software and hardware reliability in general. 


\subsection{Hardware Reliability}

Let $\mathrm{T}$ be the time representing the life of a system, and $\mathrm{t}$ as time less than $\mathrm{T}$, then the probability that the system will fail by time $t$ is given by: $F(t)=P[t \leq T]=\int_{0}^{t} f(t) d t$

Here, $f(t)$ represents the probability (or failure) density function and $F(t)$ the cumulative distribution function. The reliability function, i.e., the probability that the system survives until time $t$, is defined as

$$
R(t)=P[T>t]=1-F(t)=\int_{t}^{\infty} f(t) d t
$$

In other words, $R(t)$ represents the probability that the system will not fail by time $t$ assuming it is fault-free at time 0 .

The expected life E[T], or Mean Time to Failure (MTTF) is simply the mean or the expected value of the failure density function:

$$
E[T]=\int_{0}^{\infty} t \cdot f(t) d t
$$

It can also be shown that

$$
E[T]=\int_{0}^{\infty} R(t) d t \text {. as integration of } \mathrm{f}(\mathrm{t}) \mathrm{dt} \text { is equal to } \mathrm{R}(\mathrm{t}) .
$$

Mean Time to Repair (MTTR) is the time during which repair or replacement is occurring. Mean Time Between Failures (MTBF) is the sum of MTTF and MTTR.

The failure rate is the probability that a failure per unit time occurs in an interval such as $[t, t+\Delta t]$, knowing that a failure has not occurred before $t 1$ :

$$
\frac{P[t \leq T<t+\Delta t \mid T>t]}{t+\Delta t-t}=\frac{P[t \leq T<t+\Delta t]}{(\Delta t) P[T>t]}=\frac{F(t+\Delta t)-F(t)}{(\Delta t) R(t)}
$$

The hazard rate, on the other hand, is defined as the limit of the failure rate as the interval approaches to zero:

$$
h(t)=\lim _{\Delta t \rightarrow 0} \frac{F(t+\Delta t)-F(t)}{\Delta t R(t)}=\frac{f(t)}{R(t)}
$$


So, there is a difference between the hazard rate and failure rate; the hazard rate is an instantaneous rate of failure at time $t$ for a system of age $t$. The hazard rate changes over the life cycle of a physical system, typically it decreases, remains constant, and then increases with time giving a "bathtub curve" as shown in Figure 3.1. The bathtub curve is the most common model utilized for hardware components. To illustrate how this function is obtained, consider a population of identical components from which we take a large sample $\mathrm{N}$ and place it in operation at time $\mathrm{T}=0$. The sample will experience a high failure rate at the beginning of the operation time due to weak or substandard components, manufacturing imperfections, design effects and installation defects. This period of decreasing failure rate is referred to as "Burn-in" region. At the end of burn-in region, the failure rate will eventually reach a constant value. During the constant failure-rate region, the failures do not follow a predictable pattern but occur at random due to the changes in the applied load. This region is called as "useful life" region. The third and final region of the failure-rate curve is the "wear-out" region. The beginning of wear out region is noticed when the failure rate start to increase significantly more than the useful life value, and the failures are no longer attributed to randomness but are due to the age and wear of the components [9].

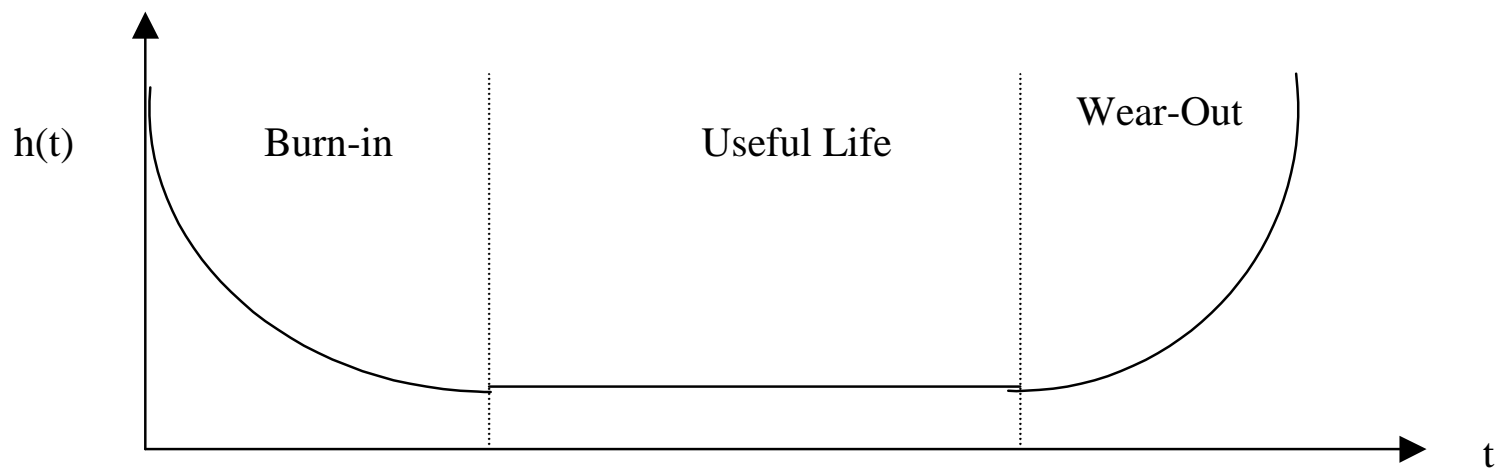

Figure 3.1: The Bathtub Curve

\subsection{Types of Distributions}

Failure data enable one to fit a model for a failure distribution. Some of the common distributions for hardware components are described below.

\subsubsection{Normal Distribution}


The Normal distribution is often used as the lifetime distribution, even though it allows negative values $(-\infty$ to $+\infty)$ with positive probability. The normal probability density function (PDF) can be used to represent the distribution of the times to failure when the failure mode is wear-out failure mode. The parameters of this model are mean $(\mu)$ and standard deviation $(\sigma)$. The normal PDF is given by:

$$
f(t)=\frac{1}{\sqrt{2 \Pi \sigma^{2}}} e^{-\frac{(t-m)^{2}}{2 \sigma^{2}}} \quad\left(\begin{array}{c}
-\infty \leq t \leq \infty \\
-\infty \leq m \leq \infty \\
0 \leq \sigma^{2} \leq \infty
\end{array}\right)
$$

\subsubsection{Exponential Distribution}

One of the most common failure distributions in reliability engineering is the exponential or constant failure rate (CFR) model. Failures due to completely random or chance events follow this distribution. This distribution dominates during the useful life of a system or component. It is also one of the easiest distributions to analyze statistically. The parameter of this distribution is failure rate $(\lambda)$. The probability density function for the exponential distribution is:

$$
f(t)=\lambda e^{-\lambda t} \quad\left(\begin{array}{l}
0 \leq t \leq \infty \\
0 \leq \lambda \leq \infty
\end{array}\right)
$$

\subsubsection{Weibull Distribution}

Weibull is the most commonly used probability distribution function in reliability. Weibull Distribution can model a wide variety of failure situations. The family of Weibull distributions can be written to include both increasing and decreasing failure rate. It is characterized by a hazard rate function of the form $\lambda(t)=a t^{b}$, which is a power function. The function $\lambda(t)$ is increasing for $a>0, b>0$ and is decreasing for $a>0$, $b<0$. The parameters of this distribution are shape parameter $(\beta)$, location parameter $(\gamma)$ and scale parameter $(\eta)$. The probability density function for the Weibull distribution is: 


$$
f(t)=\frac{\beta}{\eta}\left(\frac{t-\gamma}{\eta}\right)^{\beta-1} e^{\left(\frac{t-\gamma}{\eta}\right)^{\beta}} \quad(\theta>0, \beta>0, t \geq 0)
$$

\subsubsection{Log-Normal Distribution}

The positive random variable $\mathrm{t}$ has a Log-Normal distribution when logarithm of time is normally distributed. The density of the Log-Normal distribution has important property that is practically zeroed at the origin, increases to a maximum, and then decreases relatively quickly. The Log-Normal function is therefore suitable for modeling repair times. It is also used as a function for failure free operating time components in accelerated reliability testing as well as in cases where a large number of statistically independent random variables are combined together in multiplicative fashion. The parameters of this distribution are mean $\left(t_{m e d}\right)$ and standard deviation $(\sigma)$. The probability density function is:

$$
f(t)=\frac{1}{\sqrt{2 \Pi} \sigma t} e^{\left(\frac{-1}{2 \sigma^{2}} \ln \left(\frac{t}{t_{\text {med }}}\right)^{2}\right)} \quad(t \geq 0)
$$

\subsection{Data Analysis}

Failure data can be complete or censored. Here we will discuss singly censored data.

\subsubsection{Singly Censored Data}

A common problem in generating reliability data is censoring. Censoring occurs when data is incomplete because components are removed from consideration prior to their failure or because the test is completed prior to all components failing. That is, all components have the same test time, and the test is concluded before all components have failed. Components may be removed, for example, when they fail because of other failure modes than the one being measured. The singly censored data is further classified into Type I Censoring and Type II Censoring. In Type I censoring the testing is terminated after a fixed length of time has elapsed. Whereas in Type II censoring testing is terminated after a fixed number of failures occur.

Under each distribution, least squares (L-S) estimates, correlation coefficient, T- 
statistic, Maximum Likelihood Estimators (MLE) to determine reliability parameters, and goodness of fit to test whether data fits in a particular distribution or not. Brief discussions of these are as follows:

\subsubsection{Least Square Estimates}

The first order linear model is given by:

$$
\mathrm{Y}=\beta_{0}+\beta_{1} X_{i}+\varepsilon_{i}
$$

Where $\beta_{0}$ and $\beta_{1}$ are the intercept and slope respectively and $\varepsilon_{\mathrm{i}}$ is the error term. The predicted value of $\mathrm{Y}$ is:

$$
\mathrm{Y}^{\prime}=\mathrm{b}_{0}+\mathrm{b}_{1} \mathrm{X}
$$

Where $b_{0}$ and $b_{1}$ are predicted parameters. If $Y^{\prime}$ is the predicted value of $Y$ for $a$ given value of $\mathrm{X}$, then the residual is given by $\mathrm{Y}-\mathrm{Y}^{\prime}$. The method of least squares chooses the regression line $Y^{\prime}=b_{0}+b_{1} X$ that minimizes the sum of squares of residual $\Sigma\left(\mathrm{Y}-\mathrm{Y}^{\prime}\right)^{2}$ for all sample points.

\subsubsection{Correlation}

The primary purpose of linear correlation analysis is to measure the strength of a linear relationship between two variables. It is computed (for a sample of $n$ measurements on $\mathrm{X}$ and $\mathrm{Y}$ ) as follows:

$$
\operatorname{cor}=\frac{S S_{x y}}{\sqrt{S S_{x} S S_{y}}}=\frac{\operatorname{Cov}(x, y)}{\sigma_{x} \cdot \sigma_{y}}
$$

Where,

$$
\begin{array}{ll}
S S_{x}=\sum x^{2}-\frac{\left(\sum x\right)^{2}}{n} & \text { (Sum of Squares of } \mathrm{x}) \\
S S_{y}=\sum y^{2}-\frac{\left(\sum y\right)^{2}}{n} & \text { (Sum of Squares of } \mathrm{y}) \\
S S_{x y}=\sum x y-\frac{\left(\sum x\right)\left(\sum y\right)}{n} & \text { (Sum of Squares of } \mathrm{xy})
\end{array}
$$

\subsubsection{T-Statistic}

The T-Statistic is used to test for the existence of slope. The hypothesis is 


$$
\begin{aligned}
& \mathrm{H}_{0}: \beta_{1}=0 \\
& \mathrm{H}_{\mathrm{A}}: \beta_{1} \neq 0
\end{aligned}
$$

Where $\beta_{1}$ is the slope.

The test is given by:

$$
T S=\frac{b_{1}}{\left(\frac{\text { Std. Error }}{\sqrt{S S_{x}}}\right)} \quad\left(\mathrm{b}_{1}-\text { Slope of regression line }\right)
$$

Where,

Std. Error $=\sqrt{\frac{S S_{y}-b_{1}\left(S S_{x y}\right)}{d f}} \quad(\mathrm{df}-$ degrees of freedom $)$

Once the TS is calculated, the hypothesis is either accepted or rejected depending on the significance level [1].

\subsubsection{Maximum Likelihood Estimators (MLE)}

The method of maximum likelihood is a general method of finding estimators. Suppose we are sampling failure times whose probability function of the failure times is given by $\mathrm{f}\left(\mathrm{t} ; \theta_{1}, \ldots \ldots, \theta_{\mathrm{k}}\right)$. Given independent values $\mathrm{t}_{1}, \ldots \ldots, \mathrm{t}_{\mathrm{k}}$, the joint probability function of the failure times is given by:

$h\left(t_{1}, \ldots \ldots \ldots, t_{n}\right)=\prod_{i=1}^{n} f\left(t_{i} ; \theta_{1}, \ldots \ldots \ldots, \theta_{k}\right)$

When this joint probability function is viewed as a function of $\theta$, with the values given, it is called the likelihood function $\mathrm{L}(\theta)$. To find the MLE for any parameter with complete data, the maximum of the following likelihood function with respect to unknown parameters $\theta_{1}, \ldots \ldots, \theta_{\mathrm{k}}$ is found:

$$
L\left(\theta_{1}, \ldots \ldots \ldots, \theta_{k}\right)=\prod_{i=1}^{n} f\left(t_{i} ; \theta_{1}, \ldots \ldots \ldots \ldots, \theta_{k}\right)
$$

With singly censored data (Type I or Type II), the likelihood function is modified to be

$$
L\left(\theta_{1}, \ldots \ldots \ldots, \theta_{k}\right)=\prod_{i=1}^{r} f\left(t_{i} ; \theta_{1}, \ldots \ldots \ldots \ldots, \theta_{k}\right)[R(t t)]^{n-r}
$$

where $r$ is the number of failures and $n$ is the number at risk. The factor $[R(t t)]^{n-r}$ is the probability that the n-r censored units do not fail prior to the termination of the test.

When multiple censored data are present, the likelihood function must be 
modified to reflect the fact that at the censored times no failures occurred. This can be accomplished by defining the likelihood function in the following manner:

$$
L(\theta)=\prod_{i \in F} f\left(t_{i} ; \theta\right) \prod_{i \in C} R\left(t_{i}^{+} ; \theta\right)
$$

where $\theta$ is the unknown parameter, $\mathrm{F}$ is the set of indices for the failure times, and $\mathrm{C}$ is the set of indices for the censored times and $\mathrm{t}^{+}$is a censored time. For any types of data, the objective is to find the values of the estimators of $\theta_{1}, \ldots \ldots, \theta_{\mathrm{k}}$ that render the likelihood function as large as possible for given values of $t_{1}, \ldots \ldots, t_{n}$.

\subsubsection{Goodness-Of-Fit Tests}

Goodness-Of-Fit tests compare a null hypothesis with an alternative hypothesis:

$\mathrm{H}_{0}$ : The failure times came from the specified distribution.

$\mathrm{H}_{\mathrm{A}}$ : The failure times did not come from the specified distribution.

The test consists of computing a statistic based on the sample of failure times. This statistic is then compared with a critical value. The critical value depends on the level of significance of the test and the sample size. For this research, three goodness of fit tests will be used. These three tests are designed for specific distributions. For instance, Kolmogorov-Smirnov test is designed for normal and lognormal distributions, Bartlett's test is designed for exponential distribution, and Mann's test is designed for the Weibull distribution. The tests are described in Appendix I, II and III. The general

goodness of fit test can be performed by chi- square goodness-of-fit test. This can be shown as:

$$
\chi^{2}=\sum_{i=1}^{k} \frac{\left(O_{i}-E_{i}\right)^{2}}{E_{i}}
$$

where $\mathrm{k}=$ number of classes, $\mathrm{O}_{\mathrm{i}}=$ observed number of failures and $\mathrm{E}_{\mathrm{i}}=$ expected number of failures. 


\section{Chapter 4: Software Reliability}

\subsection{Introduction}

Measurement is a vital element in the practice of engineering, with reliability being one of the most important measurements. An understanding of the failure process is central to any effort to model and comprehend reliability.

Software reliability is defined as the probability of failure-free software operation for a specified period of time in a specified environment. It is one of the key attributes of software quality, a multidimensional property including other factors like functionality, usability, performance, serviceability, capability, instability, maintainability and documentation [12]. Software reliability is the key factor in software quality since it quantifies software failures, which can make a powerful system inoperative or even deadly, making it an essential ingredient in customer satisfaction.

Software reliability represents a user- (or customer-) oriented view of software quality. It relates directly to operation rather than design of the program, and hence it is dynamic rather than static. Therefore it becomes apparent that the distinction between terms "failure" and "fault" is important. For this reason software reliability is interested in failures occurring and not faults in program. Reliability measures are much more useful than fault measures. Software reliability may be expected to vary during the software development period.

A Failure occurs when the user perceives that the program ceases to deliver the expected service. In other words, failure is something dynamic, i.e., occurring at execution time. It is not a bug or fault. It is more general. For example, excessive response time may be considered as failure if it does not meet the specifications.

A Fault is uncovered when either a failure of the program occurs or an internal error (e.g., an incorrect state) is detected within the program. The cause of failure or internal error is said to be a fault. It is also referred to as a bug. A fault may result from an error made by the programmer. Errors occur because of (a) lack of communication between the people involved in a project or between different times for the same person; (b) incomplete knowledge of the application area, the design methodology, and the programming language; (c) incomplete analysis of the possible conditions that can occur at a given point in the program; and (d) transcription errors.

A random process is represented as a set of random variables, each 
corresponding to a point in time. In reliability studies there are two characteristics of a random process: (a) the probability distribution of the random variables, e.g., Poisson; and (b) the variation of the process with time. A random process whose probability distribution varies with time is called non-homogeneous. Two functions can be defined for the time variation of a random process: (a) the mean value function, $\mu$, as the average cumulative failures associated with each time point; and (b) the failure intensity function, $\lambda$, as the rate of change of mean value function or the number of failures per unit time. Note that the failure intensity function is the derivative of the mean value function.

The first study on software reliability appears to have been conducted by Hudson[13]. He reviewed software development as a birth and death process. Where Fault generation being a birth and fault correction being a death. He generally confined his work to pure death process, for reasons of mathematical tractability. The number of faults detected follows binomial distribution whose mean value function of time has a Weibull distribution. Data from the system test phase of one program was presented. Reasonable agreement between model and data was obtained if the system test phase is spilt into three overlapping sub-phases and separate fits made for each.

The next major steps were made by Jelinski and Moranda[15] and Shooman[28]. Both assumed hazard rate for failures to be piecewise constant and proportional to the number of faults remaining. The hazard rate changes at each fault correction by constant amount but is constant between corrections. Shooman[28] postulated that hazard rate was proportional to fault density per instruction, the number of unique instructions executed per unit time, and a bulk constant. The bulk constant represented the proportion of faults that cause failure.

Schick and Wolverton[29] proposed another early model. The hazard rate assumed was proportional to the product of the number of faults remaining and the time. Hence, the size of the changes in hazard rate increase with time. Wagoner[34] suggested a model in which the hazard rate was proportional to the number of faults remaining and power of time. This power could be varied to fit the data. In 1978 Schich and Wolverton[30] proposed modified model in which hazard rate is a parabolic function instead of linear function of time. Schneidewind[31] initially approached software reliability modeling from empirical viewpoint. He recommended the investigation of different reliability functions and selection of the distribution that best fit the particular 
project in question. In a later paper, Schneidewind[32] viewed fault detection per time interval as a Non-Homogeneous Poison Process (NHPP) with an exponential mean value function. He also suggested that the time lag between failure detection and failure correction be determined from actual data and used to correct the time scale in forecast.

Moranda[18] proposed two variants of the Jelinski - Moranda model. In "geometric de-eutrophication process", the hazard rate decreases in steps that form a geometric progression. The second, called the Geometric Poisson Model (GPM), has a hazard rate that also decreases in geometric progression. However, the decrements occur at fixed intervals rather that at each failure correction.

A Bayesian approach to software reliability measurement was taken by Littlewood and Verall[17]. Almost all published models assume that failures occur randomly during the operation of program. However, while most postulate simply that the value of hazard rate is a function of number of faults remaining, Littlewood and Verrall modeled it as a random variable. One of the parameters of the distribution of this random variable is assumed to vary with the number of failures experienced. It thus characterizes reliability change. Littlewood and Verrall proposed various functional forms for the description of this variation. The values of the parameters of each functional form that produce best fit for that form is determined. Then functional forms are compared and the best fitting form is selected.

It is seen that much of the early history of software reliability modeling involved looking at different possible models. In the late 70's and 80's, efforts began to focus on comparing software reliability models, with the objective of selecting the best ones. The initial efforts at comparison suffered from lack of good failure data and lack of agreement on the criteria to be used in making comparisons. Examination of the basic concepts underlying software reliability modeling and development of classification scheme has helped to clarify and organize comparisons and to suggest possible new models. This work led to the development of Musa-Okumoto[20] Logarithmic-Poisson execution time model, which combines simplicity, with high predictive validity. The Logarithmic Poisson model is based on NHPP with an intensity function that decreases exponentially with expected failures experienced.

In late 90's efforts began to focus on tools available for reliability estimation and to handle failure data. Yuan [35] presents a study which discussed notion of discrete- 
time software reliability modeling and distinguishing of types of software run reliability data.

\subsection{Software Reliability Estimation}

Software reliability estimation is the application of statistical inference procedures to failure data taken from software testing and operation to determine software reliability. There are four major components in the software reliability estimation process, namely

i. Reliability objective

ii. Operational profile.

iii. Reliability modeling and measurement.

iv. Validation.

At first quality of software is quantitatively defined from customers' point of view by defining failures and failure severity, by determining a reliability objective and specifying balance among key quality objectives (e.g., reliability delivery cost). Second customer usage is quantified by developing an operational profile. Operational profile is set of disjoint alternatives of system operation and their associated probabilities of occurrence. The construction of operational profile encourages testers to select test cases according to the systems operational usage, which contributes to more estimation of software reliability in the field [1]. The entire framework of software reliability estimation is shown in Figure 4.1. 


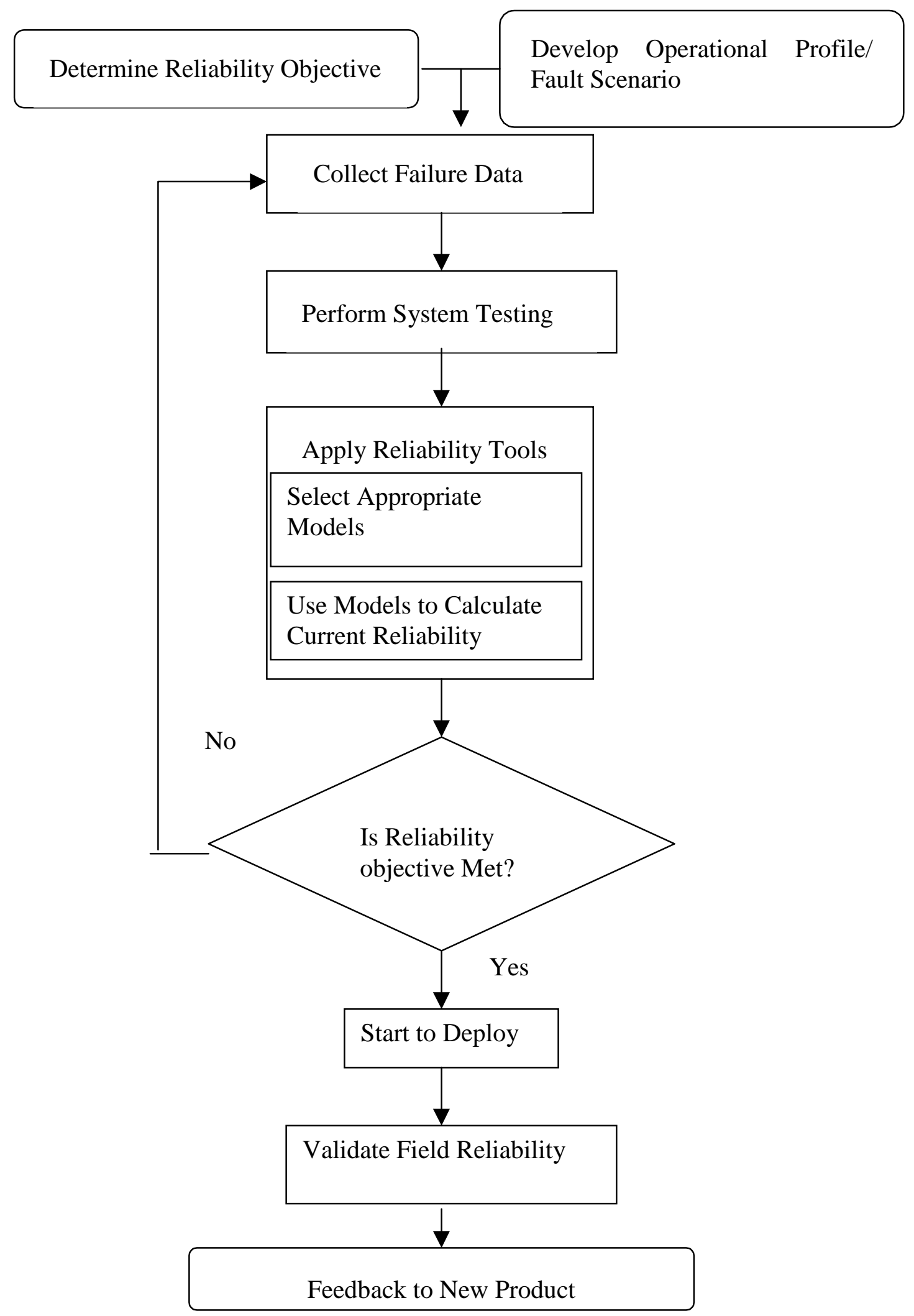

Figure 4.1: Software Reliability Measurement Procedure Overview 
Reliability quantities have usually been defined with respect to time, although it is possible to define them with respect to other variables. Time may be considered as:

a) Execution time $(\tau)$, i.e., CPU time;

b) Calendar time; and

c) Clock time, i.e., the sum of times passed from program start to program end, without counting shut shown periods.

Execution time is considered superior to calendar time [19].

There are four general ways of characterizing failure occurrences in time:

1. Time of failure

2. Time interval between failures (incremental) $\}$ Time-based

3. Cumulative failures experienced up to given time.

4. Failures experienced in a time interval.

Failure-based

The above four quantities are in fact random variables, because, a) the locations of faults within a program are unknown; and b) the conditions of program execution are generally unpredictable

When there are no changes in the software, i.e., no debugging and software corrections take place, then failure rate $(\lambda)$ is constant and a homogeneous random process takes place. On the contrary, when software corrections occur, a nonhomogeneous process as described before takes place. Figure 4.2 illustrates the mean value and related failure intensity functions of such a process. These graphs are typical in the sense that the mean number of failures experienced increases with time in such a manner that the failure intensity decreases.
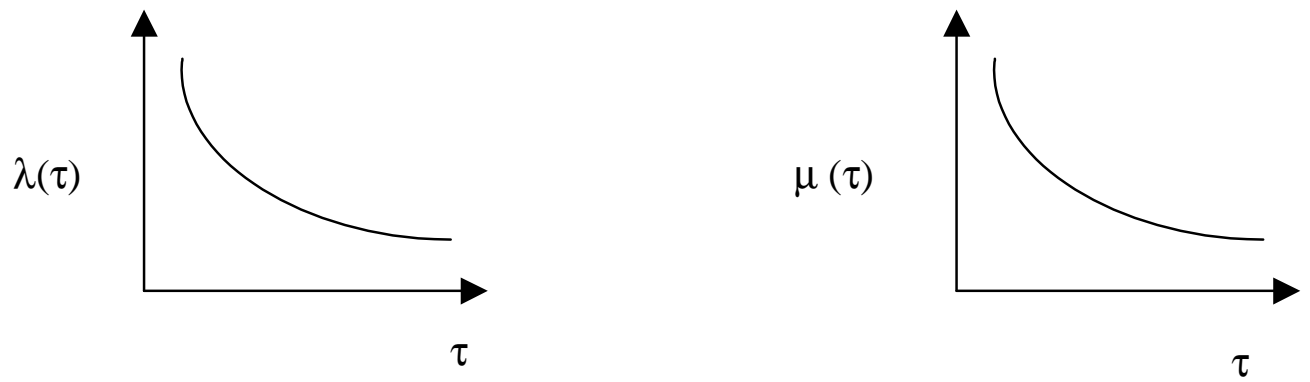

\section{Figure 4.2: Failure Intensity Curve and Mean Value Function for a Non- Homogeneous Process}

This behavior is affected by two factors: (a) the number of faults in the software; and (b) the execution environment. 
Let $\mathrm{M}(\tau)$ be a random process representing the number of failures experienced by time $\tau$, then the mean value function is defined as $\mu(\tau)=E[M(\tau)]$ i.e., expected number of failures at time $\tau$. The failure intensity function of the $M(\tau)$ processes is the instantaneous rate of change of the expected number of failures with respect to time, or

$$
\lambda(\tau)=\frac{d \mu(\tau)}{d \tau}
$$

The "time" used here may be any one of the above-mentioned three times, but execution time is generally preferred in order to be compatible with hardware reliability.

Principal factors affecting software reliability are fault introduction, fault removal, and the environment [19]. Fault introduction depends on the characteristics of the code developed, i.e., created or modified - such as its size - and of the development process - such as software engineering technology and tools used and level of experience of programmers. Fault removal depends on time, operational profile, and the quality of repair activity. Environment is determined by the operational profile, which is the set of run types that a program can execute along with the probabilities with which they will occur. It is generally established by enumerating the possible input states and their probabilities of occurrence or by specifying the sequence of program modules executed.

As faults are removed, as in the test phase, failure intensity tends to decrease and reliability tends to increase. When faults are introduced during operation or test, as in cases when new features or design changes are being introduced into the system or when faults predominate repairs during debugging, there tends to be a step increase in failure intensity and a step decrease in reliability. If a system is stable, and in a program that has been released, there are no changes in code, both failure intensity and reliability tend to be constant.

The term Mean Time To Failure (MTTF), which means the average value of next failure interval, is not used as extensively in software reliability as in hardware reliability, since in many cases it is undefined. Instead, failure intensity, which is the inverse of MTTF, is preferred. 


\subsection{Finite Failure Category Models}

The finite failure category models have fixed number of faults. The fault concept is often useful for developing finite category models because of the physical reality of defects in program. The models are classified according to how the failure quantity distribution is specified. Poisson and binomial are the most important model in this group. Binomial type models have deterministic number of faults and failures and one fault is removed for each failure. Poisson type models have random number of failures and the number of faults removed for each failure is a random variable. The models under this category are:

1. Schick-Wolverton model: Here the failure distribution type is Weibull distribution.

2. Basic Musa model: Here the failure distribution is of exponential type.

3. S-Shaped model: In this model failure distribution follows Gamma distribution.

\subsubsection{Schick-Wolverton Model}

In this model failure distribution follows Weibull distribution. This model belongs to finite failures category and is of the binomial type. This model has greater flexibility for the failure modeling because of the shape and scale parameters that define them. Basic assumptions are as follows:

- There are a fixed number of faults in the software.

- The time to failure of fault b0 (location parameter) is distributed as a Weibull distribution with parameter $\mathrm{b} 1$ and $\mathrm{b} 2$ (shape and scale parameter). The density function is $f(\tau)=b 0 * b 1 * \tau^{b 2-1} * e^{-b 1^{*} \tau^{b 2}}$ with $\mathrm{b} 1$, b2 $>0$. For Schick Wolverton model value of b2 is 2 .

- The number of faults $\left(f_{1}, f_{2}, \ldots, f_{n}\right)$ detected in each of the respective intervals $\left[\left(t_{0}=0, t_{1}\right),\left(t_{1}, t_{2}\right) \ldots\left(t_{i-1}, t_{i}\right) \ldots\left(t_{n-1}, t_{n}\right)\right]$ are independent for any finite collection of times.

The data requirements to implement this fault count model are:

- The fault counts in each of the testing intervals i.e., the $\mathrm{f}_{\mathrm{I}}$, and the completion time of each period that the software is under observation i.e., $\mathrm{t}_{\mathrm{i}}$ 's.

The failure intensity and mean value function is given by:

$$
\lambda(\tau)=b 0 * b 1 * \tau^{b 2-1} * e^{-b 1 * \tau^{b 2}} \quad \mu(\tau)=b 0 *\left(1-e^{-b 1 * \tau^{b 2}}\right)
$$




\subsubsection{Basic Musa Model}

The Basic Musa model has the widest distribution among the software reliability models and was developed by John Musa of AT\&T Bell Laboratories [19]. Musa has been a leading contributor in this field and has been a major proponent of using models to aid in determining the reliability of software. This model was one of the first to use the actual execution time of the software component on a computer for the modeling process. The basic assumptions and the data requirements for the model are as follows:

1. The cumulative number of failures by time $\tau, \mu(\tau)$, follows a Poisson process with mean value function $\mu(\tau)=\mathrm{b} 0 *\left(1-\mathrm{e}^{-\mathrm{b} 1 * \tau}\right)$, where, b0, b1>0 mean value function is such that the expected number of failure occurrences for any time period is proportional to the expected number of undetected faults at that time. Since $\lim _{\tau \rightarrow \infty} \mu(\tau)=\lim _{\tau \rightarrow \infty} b 0 *\left(1-e^{-b 1 * \tau}\right)$ is a finite failure model. The parameter $\mathrm{b} 0$ is the total number of faults that would be detected in that limit.

2. The execution times between failures are piecewise exponentially distributed, i.e., the hazard rate for a single fault is constant. This is why this model belongs to the exponential class.

3. The quantities of the resources (number of fault-identification, correction personnel and computer times) that are available are constant over a segment for which the software is observed.

4. Fault-identification personnel are fully utilized and computer utilization is constant.

5. Fault-correction personnel utilization is established by the limitation of fault queue length for any fault-correction person. Fault queue is determined by assuming that fault correction is a Poisson process and that servers are randomly assigned in time.

Assumptions 3 through 5 are needed only if the second component of the basic execution model linking execution time and calendar time is desired.

Data requirements to implement this fault count model is either the actual times that the software failed, $\mathrm{t}_{1}, \mathrm{t}_{2}, \ldots, \mathrm{t}_{\mathrm{n}}$ or the elapsed time between failures $\mathrm{x}_{1}, \mathrm{x}_{2}, \ldots, \mathrm{x}_{\mathrm{n}}$, where $\mathrm{x}_{\mathrm{i}}=$ $\mathrm{t}_{\mathrm{i}}-\mathrm{t}_{\mathrm{i}-1}$.

The failure intensity and the mean value function are given as:
$\lambda(\tau)=b 0 * b 1 * e^{-b 1 * \tau}$
$\mu(\tau)=b 0 *\left(1-e^{-b 1^{*} \tau}\right)$ 


\subsubsection{S-Shaped Model}

The S-Shaped reliability growth model falls under the gamma distribution class. Here the per-fault failure distribution is gamma. The number of failures per time period is a Poisson type. It is finite model, i.e., $\lim _{\tau \rightarrow \infty} \mu(\tau)<\infty$. It is patterned, as the mean value function is often a characteristic S-shaped. The software error detection process can be described as an S-shaped growth curve to reflect the initial learning curve at the beginning, as test team members become familiar with the software, followed by growth and then leveling off as the residual faults become more difficult to uncover. The basic assumptions and the data requirement for the model is given as:

1. The cumulative number of failures by time $\tau, \mu(\tau)$, follows a Poisson process with mean value function $\mu(\tau)=\mathrm{b} 0 *\left(1-\left((1+\mathrm{b} 1 * \tau) * \mathrm{e}^{-\mathrm{b} 1 * \tau}\right)\right)$, where, b0, b1>0. This is a bounded, non-decreasing function of time with limit $\lim _{\tau \rightarrow \infty} \mu(\tau)=b 0$, which is less than infinity, i.e., it is a finite failure model.

2. The time between failures of the $(i-1)^{\text {th }}$ and the $i^{\text {th }}$ failure depends on the time to failure of the $(i-1)^{\text {th. }}$

3. When a failure occurs, the fault, which caused it, is immediately removed and no other faults are introduced.

Data requirements to implement this model are:

- The failure times, $\mathrm{t}_{\mathrm{i}}$ 's, of the software system, or

- The number of faults detected, $f$, in each period of observation of the software along with the associated lengths $1_{i}$ of those periods, $i=1, \ldots n$.

If data of type I are available, the data of second type can be constructed by first forming a partition of the time period over which the software is observed and then counting up the number of faults that fall in each respective period of partition. As a consequence, this model can be used for either the time between failure data or the number of faults per time period.

The failure intensity and mean value function is given by:

$$
\lambda(\tau)=b 0 * b 1^{2} * \tau * e^{-b 1^{*} \tau} \quad \mu(\tau)=b 0 *\left(1-\left((1+b 1 * \tau) * e^{-b 1^{*} \tau}\right)\right)
$$




\subsection{Infinite Category Model}

For infinite failures category models the number of failures in infinite time is unbounded. That is for these models $\lim _{\tau \rightarrow \infty} \mu(\tau)=\infty$ for the mean value function of the process. This means that software will never be completely fault free. This could be by additional faults being introduced in the software through the error correction process. This category includes following three models:

1. Duane's Model: This model assumes power distribution for the failure data.

2. Logarithmic Poisson Model: This model assumes geometric distribution for the failure data.

3. Inverse Linear Model: This model assumes inverse linear distribution for the failure data.

\subsubsection{Duane's Model}

At General Electric, Duane noticed that if the cumulative failure rate versus the cumulative testing time was plotted on Log-Log paper, it tended to follow a straight line [6]. This process is a non-homogeneous Poisson process in which the failure intensity function has the same form as the hazard rate for a Weibull distribution. This model is sometimes referred to as the power model since the mean value function for the cumulative number of failures by time $\tau$ is taken as a power of $\tau$, that is, $\mu(\tau)=b 0 * \tau^{b 1}$ for some, $\mathrm{b} 0>0$ and $\mathrm{b} 1>0$. This model is an infinite failure model as $\lim _{\tau \rightarrow \infty} \mu(\tau)=\infty$. The basic assumptions and data requirements are:

1. The cumulative number of failures by time $t, M(t)$, follows a Poisson process with mean value function, $\mu(\tau)=b 0 * \tau^{b 1}$ for some, $b 0>0$ and $b 1>0$.

The data requirements to implement this fault count model are:

- Either the actual times that the software failed, $t_{1}, t_{2}, \ldots, t_{n}$ or the elapsed time between failures $\mathrm{x}_{1}, \mathrm{x}_{2}, \ldots, \mathrm{x}_{\mathrm{n}}$, where $\mathrm{x}_{\mathrm{i}}=\mathrm{t}_{\mathrm{i}}-\mathrm{t}_{\mathrm{i}-1}$ and $\mathrm{t}_{0}=0$.

The failure intensity and mean value functions are:
$\lambda(\tau)=b 0 * b 1 * \tau^{b 1-1}$
$\mu(\tau)=b 0^{*} \tau^{b 1}$ 


\subsubsection{Logarithmic Poisson Model}

The logarithmic Poisson model proposed by Musa and Okumoto [20] is another model that has been extensively applied. It is also a NHPP with an intensity function that decreases exponentially as failures occur. The exponential rate of decrease reflects the view that the earlier discovered failures have a greater impact on reducing the failure intensity function than those encountered later. It is called logarithmic because the expected number of failures over time is a logarithmic function. The assumptions and data requirements are:

1. The failure intensity decreases exponentially with the expected number of failures experienced, that is, $\lambda(\tau)=\frac{b 0 * b 1}{1+b 1 * \tau}$.

2. The cumulative number of failures by time $\tau, \mu(\tau)$ follows a Poisson process.

Because of assumption 1, it follows that $\mu(\tau)=b 0 * \ln (1+b 1 * \tau)$ and therefore it is clear that this is an infinite failure model.

Data requirements are:

- Either the actual times that the software failed, $t_{1}, t_{2}, \ldots, t_{n}$ or the elapsed time between failures $x_{1}, x_{2}, \ldots, x_{n}$, where $x_{i}=t_{i}-t_{i-1}$.

The failure intensity and mean value function are as:

$\lambda(\tau)=\frac{b 0 * b 1}{1+b 1 * \tau} \quad \mu(\tau)=\mathrm{b} 0 * \ln (1+\mathrm{b} 1 * \tau)$

\subsubsection{Inverse Linear Model}

This model is a special case of Littlewood Verrall Model [17]. This is also a type of infinite failure model as $\lim _{\tau \rightarrow \infty} \mu(\tau)=\infty$. Data requirements for the model is:

Either the actual times that the software failed, $t_{1}, t_{2}, \ldots, t_{n}$ or the elapsed time between failures $x_{1}, x_{2}, \ldots, x_{n}$, where $x_{i}=t_{i}-t_{i-1}$.

The failure intensity and mean value is given by:

$$
\lambda(\tau)=b 0 * \frac{1}{2 * \sqrt{b 1+\tau}} \quad \mu(\tau)=b 0 *(\sqrt{b 1+\tau}-\sqrt{b 1}
$$




\section{Chapter 5: System Reliability}

\subsection{Introduction}

To determine the reliability of a large system, it needs to be subdivided into smaller subsystems and components whose individual reliability factors are known or can be easily determined. Depending on the manner in which these subsystems and components are connected to constitute the given system, the combination rules of probability can be applied to obtain system reliability. From the point of view of interconnection of the subsystems, a system may be classified as series, parallel, seriesparallel, or a complex system.

Finding the exact reliability for series and parallel networks is quite straightforward and is described briefly in next two sections. A series-parallel network consists of distinct series and parallel components within the given system. For such a system the reliability analysis is performed in steps as described below.

A complex system is one, which cannot be completely decomposed into independent sections of series and/or parallel sub-systems. Reliability analysis for such systems is non-trivial. As a result, many algorithms resort to simplifying assumptions that produce approximate solutions [7].

\subsection{Series System}

Consider a simple system consisting of $\mathrm{n}$ software or hardware components connected in series as shown in the Figure 5.1.

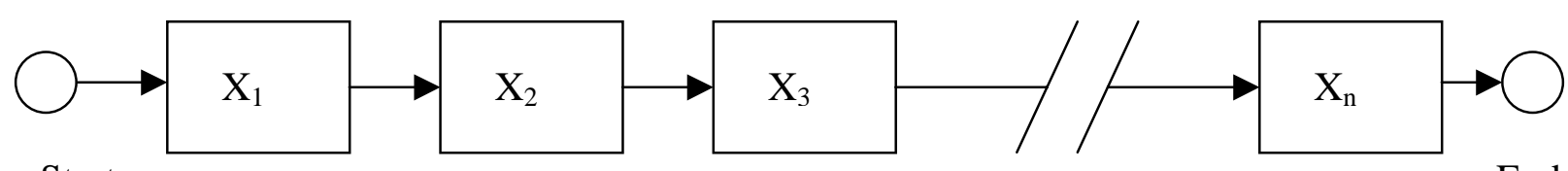

Start

End

Figure 5.1: Series System

Let $\mathrm{P}\left(\mathrm{X}_{\mathrm{i}}\right)$ represent the probability of successful operation of component $\mathrm{X}_{\mathrm{i}}$. For this series system the system reliability is given by:

$$
\begin{aligned}
& \mathrm{R}_{\mathrm{s}}=\mathrm{P}\left(\mathrm{X}_{1} * \mathrm{X}_{2} * \ldots \mathrm{X}_{\mathrm{n}}\right) \\
& \mathrm{P}\left(\mathrm{X}_{1}\right) * \mathrm{P}\left(\mathrm{X}_{2} \mid \mathrm{X}_{1}\right) * \mathrm{P}\left(\mathrm{X}_{3} \mid\left(\mathrm{X}_{1} * \mathrm{X}_{2}\right)\right) \ldots \mathrm{P}\left(\mathrm{X}_{\mathrm{n}} \mid\left(\mathrm{X}_{1} * \mathrm{X}_{2} * \ldots \mathrm{X}_{\mathrm{n}-1}\right)\right)
\end{aligned}
$$

Where $\mathrm{P}\left(\mathrm{X}_{2} \mid \mathrm{X}_{1}\right)$ means the probability of successful operation of component $\mathrm{X}_{2}$ 
under the condition that component $\mathrm{X}_{1}$ operates successfully. Likewise, $\mathrm{P}\left(\mathrm{X}_{\mathrm{n}} \mid \mathrm{X}_{1}\right.$ and $X_{2}$ and $\left.\ldots X_{n-1}\right)$ ) means the probability of successful operation of component $X_{n}$ under the condition that all remaining units are working successfully. If the successful operations of all these units are independent, the above expression becomes:

$$
R_{s}=\prod_{i=1}^{n} P\left(X_{i}\right)
$$

\subsection{Parallel Systems}

Consider another simple system consisting of $\mathrm{n}$ components connected in parallel as shown in Figure 5.2.

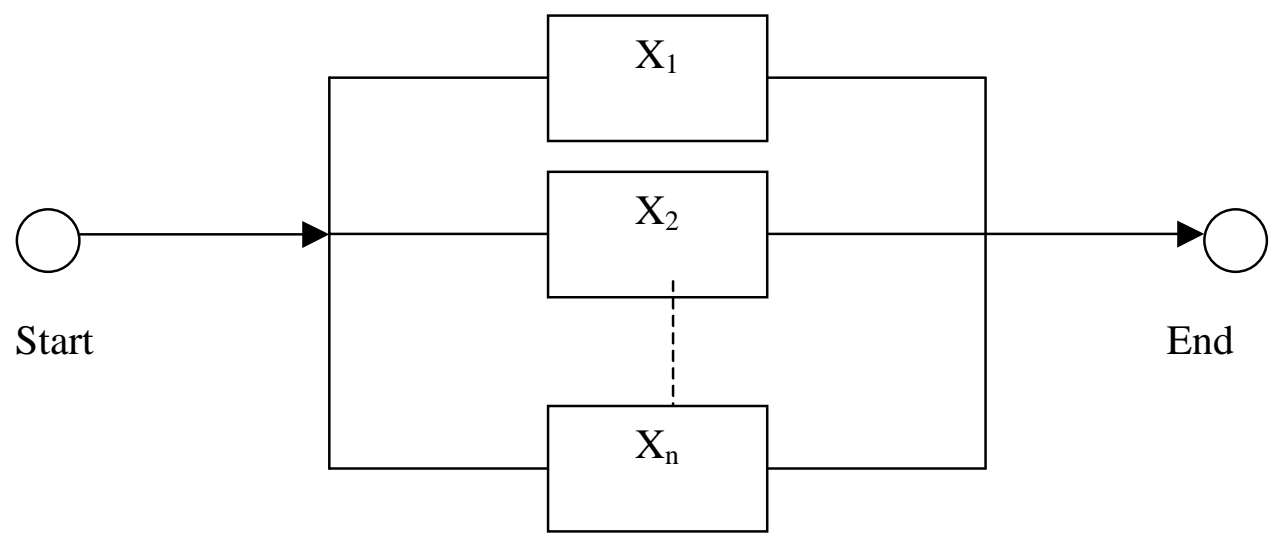

Figure 5.2: Parallel System

In parallel systems, as opposed to series system, several single path perform the same operation. The satisfactory performance of any of these paths is sufficient to ensure the successful operation of the system. Let $\mathrm{P}(\mathrm{Xi})$ represent the probability of successful operation of component $\mathrm{Xi}$. Similarly let $\mathrm{P}\left(\mathrm{Xi}^{\prime}\right)$ represent the probability of failure of component Xi. For this parallel system to fail, all of the $\mathrm{n}$ components must fail. Hence the unreliability of the system is given by:

$$
\begin{aligned}
\mathrm{Q}_{\mathrm{s}} & =\mathrm{P}\left(\mathrm{X}_{1}^{\prime} * \mathrm{X}_{2}^{\prime} * \ldots \mathrm{X}_{\mathrm{n}}\right) \\
& =\mathrm{P}\left(\mathrm{X}_{1}^{\prime}{ }_{1}\right) * \mathrm{P}\left(\mathrm{X}_{2}^{\prime} \mid \mathrm{X}_{1}^{\prime}{ }_{1}\right) \mathrm{P}\left(\mathrm{X}_{3}^{\prime} \mid\left(\mathrm{X}_{1}^{\prime} * \mathrm{X}_{2}^{\prime}\right)\right) \ldots \mathrm{P}\left(\mathrm{X}_{\mathrm{n}}^{\prime} \mid\left(\mathrm{X}_{1}^{\prime} * \mathrm{X}_{2}^{\prime} * \ldots \mathrm{X}_{\mathrm{n}-1}^{\prime}\right)\right)
\end{aligned}
$$

Where $\mathrm{P}\left(\mathrm{X}_{2}^{\prime} \mid \mathrm{X}_{1}^{\prime}\right)$ means the probability of failure of unit $\mathrm{X}_{2}$ under the condition that unit $\mathrm{X}_{1}$ also fails. Likewise, $\mathrm{P}\left(\mathrm{X}_{\mathrm{n}}^{\prime} \mid\left(\mathrm{X}_{1}^{\prime} * \mathrm{X}_{2}^{\prime} * \ldots \mathrm{X}_{\mathrm{n}-1}^{\prime}\right)\right)$ means the probability of failure of unit $X_{n}$ under the condition that all remaining units have failed. If the failures of all these units are independent, then 


$$
Q_{s}=\prod_{i=1}^{n} P\left(X_{i}^{\prime}\right)
$$

The system reliability, $\mathrm{R}_{\mathrm{s}}$, is given by

$$
R_{s}=1-\prod_{i=1}^{n} P\left(X_{i}^{\prime}\right)
$$

\subsection{Series-Parallel System}

An example of a series-parallel system is shown in Figure 5.3.

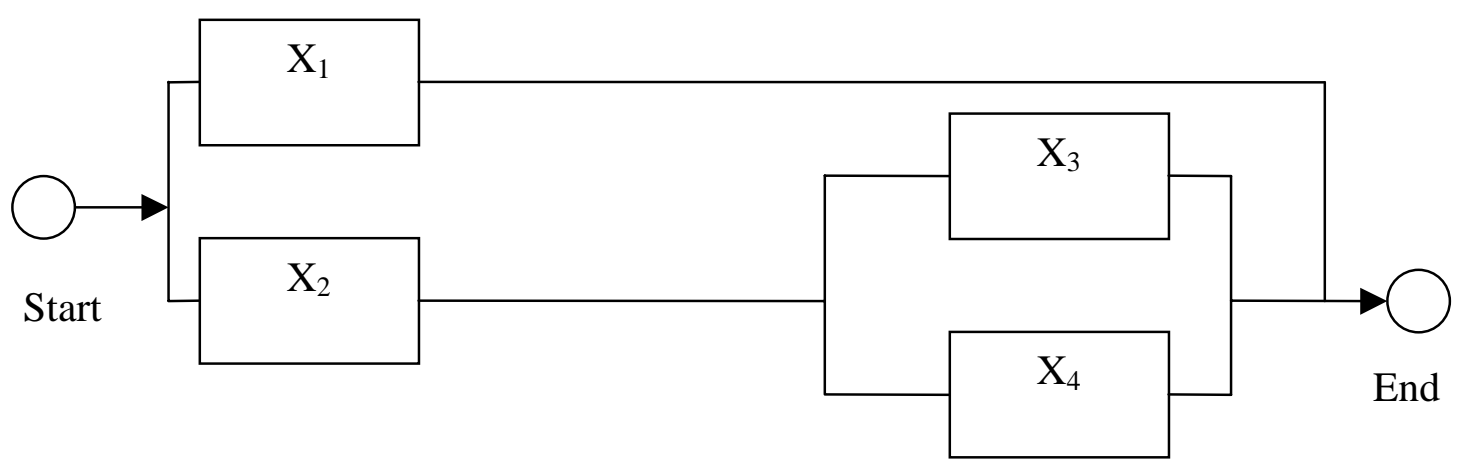

Figure 5.3: Series-Parallel System

Reliability analysis of such system is performed in steps. In each step the independent series and parallel structures are identified and solved separately. As a result of each step, the size of the system reduces until it becomes a simple series or parallel system. In the system shown in Figure 5.3, components $\mathrm{X}_{3}$ and $\mathrm{X}_{4}$ are in parallel and thus form a subsystem identified as subsystem 1 and shown in Figure 5.4.

Using the expression derived for parallel systems, and assuming unit failures as independent events, the reliability of subsystem 1 is found as:

Rsubsys1 = 1-[1-P( $\left.\left.\mathrm{X}_{3}\right)\right]^{*}\left[1-\mathrm{P}\left(\mathrm{X}_{4}\right)\right]$

In the next step, note that unit $X_{2}$ is in series with the subsystem 1. Let the unit $X_{2}$ and subsystem 1 form a bigger subsystem, which is identified as subsystem 2 and shown in Figure 5.5. Again, assuming the failure of each unit as an independent event, the reliability of subsystem 2 is given by: 


$$
\text { Rsubsys } 2=\mathrm{P}\left(\mathrm{X}_{2}\right) *\left[1-\left\{1-\mathrm{P}\left(\mathrm{X}_{3}\right)\right\} *\left\{1-\mathrm{P}\left(\mathrm{X}_{4}\right)\right)\right]
$$

Finally $\mathrm{X}_{1}$ can be combined in parallel with subsystem 2 . The reliability of the overall system is:

$\mathrm{R}_{\mathrm{S}}=1-\mathrm{P}\left(\mathrm{X}_{1}\right) *\{$ Probability of Failure of Subsystem 2$\}$

$\mathrm{R}_{\mathrm{s}}=1-\left\{1-\mathrm{P}\left(\mathrm{X}_{1}\right)\right\} *\left[1-\mathrm{P}\left(\mathrm{X}_{2}\right) *\left\{1-\left(1-\mathrm{P}\left(\mathrm{X}_{3}\right)\right\} *\left\{1-\mathrm{P}\left(\mathrm{X}_{4}\right)\right\}\right]\right.$

Assuming that all units are identical, i.e. $\mathrm{P}\left(\mathrm{X}_{1}\right)=\mathrm{P}\left(\mathrm{X}_{2}\right)=\mathrm{P}\left(\mathrm{X}_{3}\right)=\mathrm{P}\left(\mathrm{X}_{4}\right)=\mathrm{p}$ and $\mathrm{P}\left(\mathrm{X}^{\prime}{ }_{1}\right)=$ $\mathrm{P}\left(\mathrm{X}_{2}{ }_{2}\right)=\mathrm{P}\left(\mathrm{X}_{3}{ }_{3}\right)=\mathrm{P}\left(\mathrm{X}_{4}{ }_{4}\right)=1-\mathrm{p}$,

$\mathrm{R}_{\mathrm{s}}=\mathrm{p}+2 \mathrm{p}_{2}+3 \mathrm{p}_{3}+\mathrm{p}_{4}$

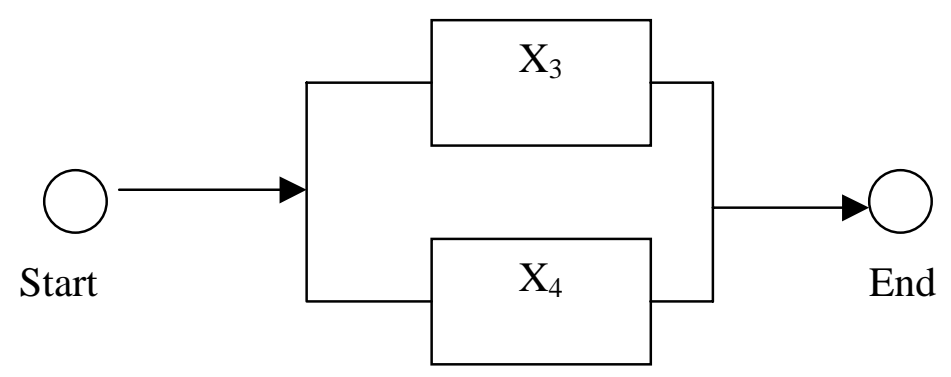

Figure 5.4: Subsystem 1

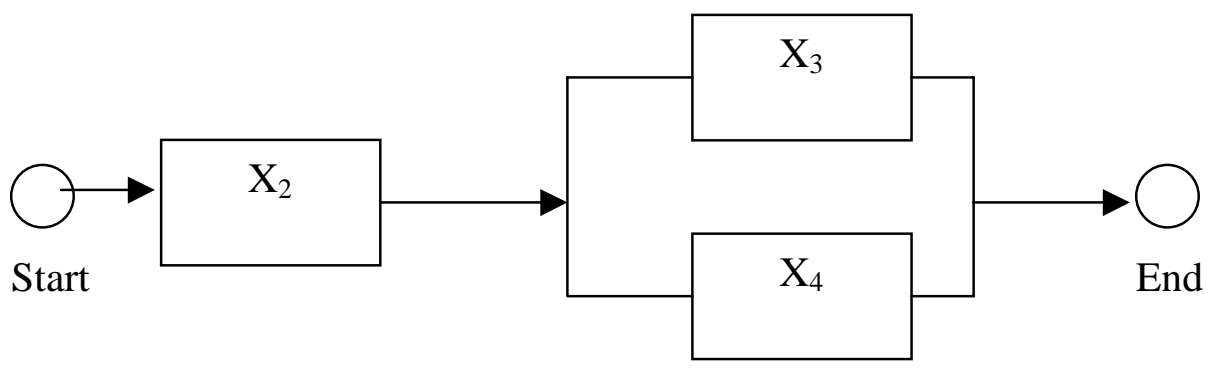

Figure 5.5: Subsystem 2

\section{5 k-out-of-n System}

The k-out-of-n system has a total of $\mathrm{n}$ components connected in parallel, and at least $\mathrm{k}$ components must operate for the system to function. Obviously $\mathrm{k} \leq \mathrm{n}$. if $\mathrm{k}=1$, complete redundancy occurs, and if $\mathrm{k}=\mathrm{n}$ then we have an $\mathrm{n}$ component series system. The reliability for such a system can be obtained from the binomial probability 
distribution.

$$
R_{s}=\sum_{j=k}^{n}\left(\frac{n !}{j !(n-j) !}\right) * R^{j} *(1-R)^{n-j}
$$

where $\mathrm{R}$ is the component reliability.

\subsection{State Dependent Systems}

A fundamental computation in reliability engineering is the determination of system reliability from knowledge of component reliabilities and their system configuration. On the basis of the critical assumption of independent failures among components the basic equations of system reliability can be derived. However, when component failures are in some way dependent, more powerful methods, such as Markov analysis is needed. For example, when one of the components connected in parallel fails, the failure rates of the surviving components are affected [9].

\subsubsection{Markov Analysis}

Markov analysis looks at a system as being in one of several states. One possible state, for example, is that in which all the components of the system are operating. Another possible state is that in which one component has failed but the other components continue to operate. The fundamental assumption in a Markov process is that the probability that a system will undergo a transition from one state to another depends only on the current state of the system and not on any previous state of the system. In other words, transition probability is not dependent on the past (state) history of the system. This is equivalent to the memorylessness of the exponential distribution, and it is therefore not surprising that exponential times to failure satisfy this Markovian property. The state transition probability is expressed as an instantaneous (failure) rate. Assuming the process is also stationary (i.e., the transition probabilities do not change over time), the transition rates will be constant. Again, this is equivalent to assuming exponential failure times.

The methodology is explained by an example. In applying Markov analysis, we assume that each of the $\mathrm{n}$ components will be in one of the two states - operating or failed. The system state is then defined to be one of the $2^{n}$ possible combinations of 
operating and failed components. For a two-component system we define the following four system states:

Table 5.1: State Table for Two Components

\begin{tabular}{|c|l|l|}
\hline State & Component $\mathbf{X}_{1}$ & Component $\mathbf{X}_{2}$ \\
\hline 1 & $\mathrm{X}_{1}$ (Operating) & $\mathrm{X}_{2}$ (Operating) \\
\hline 2 & $\mathrm{X}_{1}{ }^{\prime}$ (Failed) & $\mathrm{X}_{2}$ (Operating) \\
\hline 3 & $\mathrm{X}_{1}$ (Operating) & $\mathrm{X}_{2}{ }^{\prime}$ (Failed) \\
\hline 4 & $\mathrm{X}^{\prime}{ }^{\prime}$ (Failed) & $\mathrm{X}_{2}{ }^{\prime}$ (Failed) \\
\hline
\end{tabular}

If the two components are in parallel (redundant), only state 4 results in a system failure. On the other hand, if the two components are in series, then states 2, 3 and 4 would constitute a failure state. The objective is to find the probability of the system being in each state as a function of time. We denote the probability of being in state 1 at time $t$ as $P_{i}(t)$. Then for a two-component series system,

$\mathrm{R}_{\mathrm{s}}(\mathrm{t})=\mathrm{P}_{1}(\mathrm{t})$

And for two-component parallel system,

$\mathrm{R}_{\mathrm{p}}(\mathrm{t})=\mathrm{P}_{1}(\mathrm{t})+\mathrm{P}_{2}(\mathrm{t})+\mathrm{P}_{3}(\mathrm{t})$

Where $R_{s}(t)$ and $R_{p}(t)$ is reliability of series and parallel system at time $t$.

Observe that the system must be in one of the four sates at any given time. Therefore,

$\mathrm{P}_{1}(\mathrm{t})+\mathrm{P}_{2}(\mathrm{t})+\mathrm{P}_{3}(\mathrm{t})+\mathrm{P}_{4}(\mathrm{t})=1$

What remains is to find $\mathrm{P}_{\mathrm{i}}(\mathrm{t}), \mathrm{i}=1,2,3,4$.

If we assume that individual components have constant failure rates $\lambda_{i}$, we can represent the two-component system using the Reliability Transition Diagram (RTD) as shown in Figure 5.6. The nodes in the figure represent the four system states, and the branches show the transition rate $\left(\lambda_{\mathrm{i}}\right)$ from one node to another. 


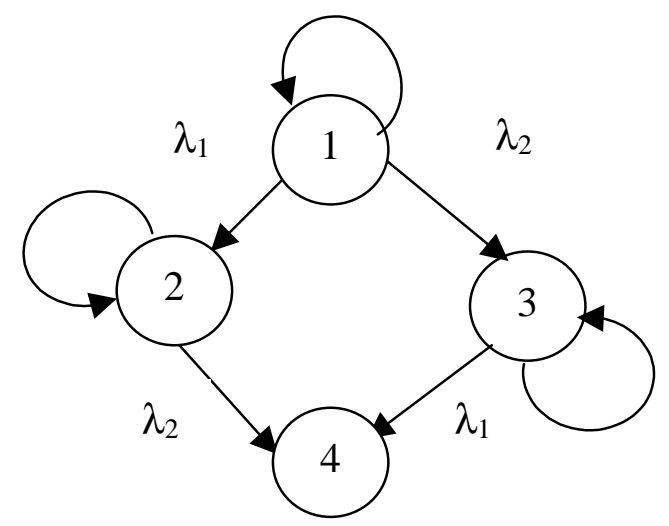

Figure 5.6: Reliability Transition Diagram

From the RTD, we can derive the following equation:

$\mathrm{P}_{1}(\mathrm{t}+\Delta \mathrm{t})=\mathrm{P}_{1}(\mathrm{t})-\lambda_{1} \Delta \mathrm{tP}_{1}(\mathrm{t})-\lambda_{2} \Delta \mathrm{tP}_{1}(\mathrm{t})$

This equation states that the probability of the system being in state 1 at time $\mathrm{t}+$ $\Delta \mathrm{t}$ is equal to the probability of it being in state 1 at time $t$ minus the probability of it being in state 1 at time t times the probability of transitioning $\left(\lambda_{i} \Delta t\right)$ to either state 2 or 3 . Observe that $\lambda_{1} \Delta \mathrm{t}$ is the conditional probability of a transition to state 2 occurring during the time $\Delta \mathrm{t}$ given that the system is currently in state 1 . Therefore, $\lambda_{1} \Delta \mathrm{tP}_{1}(\mathrm{t})$ is the joint probability of the system being in state 1 at time $t$ and making a transition to state 2 during time $\Delta \mathrm{t}$. A similar argument holds for $\lambda_{2} \Delta \mathrm{tP}_{1}(\mathrm{t})$ for transition to state 3 .

A second equation is obtained from state 2.

$\mathrm{P}_{2}(\mathrm{t}+\Delta \mathrm{t})=\mathrm{P}_{2}(\mathrm{t})+\lambda_{1} \Delta \mathrm{tP}_{1}(\mathrm{t})-\lambda_{2} \Delta \mathrm{tP}_{2}(\mathrm{t})$

is the probability of the system being in state 2 at time $t+\Delta t$ and is equal to the probability of being in state 2 at time t plus the probability of being in state 1 at time $t$ and making a transition $\left(\lambda_{1} \Delta \mathrm{t}\right)$ to state 2 in time $\Delta \mathrm{t}$ minus the probability of being in state 2 at time $\mathrm{t}$ and making a transition to state $4\left(\lambda_{2} \Delta \mathrm{t}\right)$ in time $\Delta \mathrm{t}$. Similarly for state 3 ,

$\mathrm{P}_{3}(\mathrm{t}+\Delta \mathrm{t})=\mathrm{P}_{3}(\mathrm{t})+\lambda_{2} \Delta \mathrm{tP}_{1}(\mathrm{t})-\lambda_{1} \Delta \mathrm{tP}_{3}(\mathrm{t})$

And for state 4 ,

$\mathrm{P}_{4}(\mathrm{t}+\Delta \mathrm{t})=\mathrm{P}_{4}(\mathrm{t})+\lambda_{2} \Delta \mathrm{tP}_{2}(\mathrm{t})+\lambda_{1} \Delta \mathrm{tP}_{3}(\mathrm{t})$

Rewriting Equation 5.2,

$\frac{P_{1}(t+\Delta t)-P_{1}(t)}{\Delta t}=-\left(\lambda_{1}+\lambda_{2}\right) P_{1}(t)$ 
Then

$\lim _{\Delta t \rightarrow 0} \frac{P_{1}(t+\Delta t)-P_{1}(t)}{\Delta t}=\frac{d P_{1}(t)}{d t}=-\left(\lambda_{1}+\lambda_{2}\right) P_{1}(t)$

In a similar fashion, Eqs. (5.3) and (5.4) lead to the following differential equations:

$\frac{d P_{2}(t)}{d t}=\lambda_{1} P_{1}(t)-\lambda_{2} P_{2}(t)$

$\frac{d P_{3}(t)}{d t}=\lambda_{2} P_{1}(t)-\lambda_{1} P_{3}(t)$

Equations (5.6), (5.7), and (5.8) along with equation (5.1) can be solved simultaneously.

Their solution is

$$
\begin{aligned}
& P_{1}(t)=e^{-\left(\lambda_{1}+\lambda_{2}\right) t} \\
& P_{2}(t)=e^{-\lambda_{2} t}-e^{-\left(\lambda_{1}+\lambda_{2}\right) t} \\
& P_{3}(t)=e^{-\lambda_{1} t}-e^{-\left(\lambda_{1}+\lambda_{2}\right) t} \\
& P_{4}(t)=1-P_{1}(t)-P_{2}(t)-P_{3}(t)
\end{aligned}
$$

Then for a series system we have

$$
R_{s}(t)=P_{1}(t)=e^{-\left(\lambda_{1}+\lambda_{2}\right) t}
$$

and for parallel system,

$$
\begin{aligned}
& R_{p}(t)=P_{1}(t)+P_{2}(t)+P_{3}(t) \\
& R_{p}(t)=e^{-\lambda_{1} t}+e^{-\lambda t}-e^{-\left(\lambda_{1}+\lambda_{2}\right) t}
\end{aligned}
$$

\subsubsection{Load-Sharing System}

A rather straightforward application of Markov Analysis is to a load-sharing system. There are two components in parallel and there is a dependency between them. If one component fails, the failure rate of the other component increases as a result of the additional load placed on it. Because of this dependency, the reliability block diagram techniques cannot be applied. Instead Markov analysis is used to determine the system reliability. For a two-component system the Table 5.1 shows the various states.

The RTD is shown in Figure 5.7, where $\lambda_{1}+$ and $\lambda_{2}+$ represent the increased failure rates of components 1 and 2, respectively, as a result of increased load. 


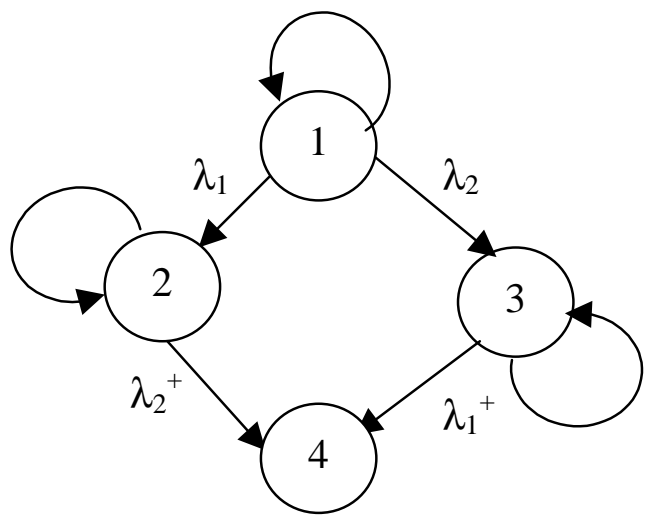

Figure 5.7: Reliability Transition Diagram for Load Sharing System

The resulting differential equations are:

$$
\begin{aligned}
& \frac{d p_{1}(t)}{d t}=-\left(\lambda_{1}+\lambda_{2}\right) p 1(t) \\
& \frac{d p_{2}(t)}{d t}=\lambda_{1} p_{1}(t)-\lambda_{2}^{+} p_{2}(t) \\
& \frac{d p_{3}(t)}{d t}=\lambda_{2} p_{1}(t)-\lambda_{1}^{+} p_{3}(t)
\end{aligned}
$$

The solutions to these equations are as follows:

$$
\begin{aligned}
& P_{1}(t)=e^{-\left(\lambda_{1}+\lambda_{2}\right) t} \\
& P_{2}(t)=\frac{\lambda_{1}}{\lambda_{1}+\lambda_{2}-\lambda_{2}{ }^{+}}\left[e^{-\lambda_{2}{ }^{+} t}-e^{-\left(\lambda_{1}+\lambda_{2}\right) t}\right] \\
& P_{3}(t)=\frac{\lambda_{2}}{\lambda_{1}+\lambda_{2}-\lambda_{1}{ }^{+}}\left[e^{-\lambda_{1}^{+} t}-e^{-\left(\lambda_{1}+\lambda_{2}\right) t}\right]
\end{aligned}
$$

and

$\mathrm{R}_{\mathrm{s}}(\mathrm{t})=\mathrm{P}_{1}(\mathrm{t})+\mathrm{P}_{2}(\mathrm{t})+\mathrm{P}_{3}(\mathrm{t})$

If we let $\lambda_{1}=\lambda_{2}=\lambda$ and $\lambda_{1}{ }^{+}=\lambda_{2}{ }^{+}=\lambda^{+}$, then

$$
R(t)=e^{-2 \lambda t}+\frac{2 \lambda}{2 \lambda-\lambda^{+}}\left[e^{-\lambda^{+} t}-e^{-2 \lambda t}\right]
$$

and

$$
M T T F=\int_{0}^{\infty} R(t) d t=\frac{1}{2 \lambda}+\frac{2 \lambda}{2 \lambda-\lambda^{+}}\left[\frac{1}{\lambda^{+}}-\frac{1}{2 \lambda}\right]
$$




\subsubsection{Standby Systems}

Standby systems are an important area of study within reliability. Depending on the probability of failure occurring when switching to a standby unit, these systems are much more reliable than the active redundant system. The two-component standby system differs form the active redundant system discussed earlier in that the standby unit will have no failures or a reduced failure rate while in its standby mode. Once active, the backup unit may experience the same failure rate as the online (primary) system (if they are identical) or may have a different failure rate. The dependency arises because the failure rate of the standby unit depends on the state of the primary unit.

The states of the system are shown in Figure 5.8. The differential equations for this system is as shown

$$
\begin{aligned}
& \frac{d p_{1}(t)}{d t}=-\left(\lambda_{1}+\lambda_{2}\right) p_{1}^{-}(t) \\
& \frac{d p_{2}(t)}{d t}=\lambda_{1} p_{1}(t)-\lambda_{2} p_{2}(t) \\
& \frac{d p_{3}(t)}{d t}=\lambda_{2}^{-} p_{1}(t)-\lambda_{1} p_{3}(t)
\end{aligned}
$$

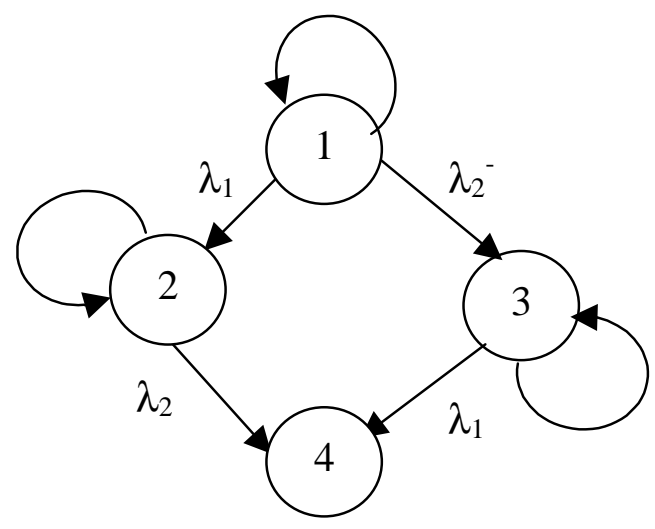

Figure 5.8: Reliability Transition Diagram for Standby System

The solutions to above differential equations are as follows:

$$
\begin{aligned}
& P_{1}(t)=e^{-\left(\lambda_{1}+\lambda_{2}^{-}\right) t} \\
& P_{2}(t)=\frac{\lambda_{1}}{\lambda_{1}+\lambda_{2}^{-}-\lambda_{2}}\left[e^{-\lambda_{2} t}-e^{-\left(\lambda_{1}+\lambda_{2}^{-}\right) t}\right] \\
& P_{3}(t)=\left[e^{-\lambda_{1} t}-e^{-\left(\lambda_{1}+\lambda_{2}^{-}\right) t}\right]
\end{aligned}
$$


and

$\mathrm{R}(\mathrm{t})=\mathrm{P}_{1}(\mathrm{t})+\mathrm{P}_{2}(\mathrm{t})+\mathrm{P}_{3}(\mathrm{t})$

$R(t)=e^{-\lambda_{1} t}+\frac{\lambda_{1}}{\lambda_{1}+\lambda_{2}{ }^{-}-\lambda_{2}}\left[e^{-\lambda_{2} t}-e^{-\left(\lambda_{1}+\lambda_{2}{ }^{-}\right) t}\right]$

and

$\operatorname{MTTF}=\frac{1}{\lambda_{1}}+\frac{\lambda_{1}}{\lambda_{2}\left(\lambda_{1}+\lambda_{2}^{-}\right)}$

If there are no failures of the standby unit, set $\lambda_{2}{ }^{-}=0$ in Eqs. (5.27) and (5.28). If $\lambda_{1}=\lambda_{2}=\lambda$ and $\lambda_{2}{ }^{-}=\lambda^{-}$, then Eqs. (5.27) and (5.28) simplify to

$R(t)=e^{-\lambda t}+\frac{\lambda}{\lambda^{-}}\left[e^{-\lambda t}-e^{-\left(\lambda+\lambda^{-}\right) t}\right]$

$M T T F=\frac{1}{\lambda}+\frac{1}{\lambda+\lambda^{-}}$

\subsubsection{Standby Systems with Switching Failures}

It is not uncommon in a standby system to have some probability $\mathrm{p}$ of there being an on-demand failure of a switching device designed to place the standby system on-line. The transition diagram is as shown in figure 5.9.

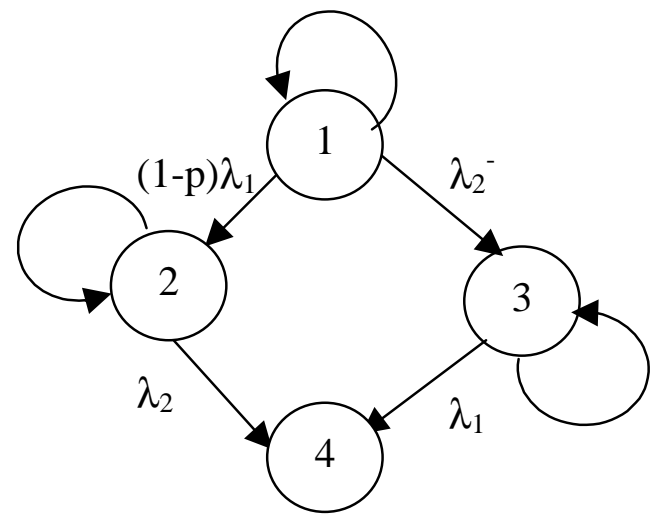

Figure 5.9: Reliability Transition Diagram for Standby Systems with Switching Failures

The reliability function is given by

$$
R(t)=e^{-\lambda_{1} t}+\frac{(1-p) \lambda_{1}}{\lambda_{1}+\lambda_{2}^{-}-\lambda_{2}}\left[e^{-\lambda_{2} t}-e^{-\left(\lambda_{1}+\lambda_{2}{ }^{-}\right) t}\right]
$$




\subsubsection{Three-Component Standby System}

Consider a system with one active component and two standby components. For simplicity assume that no component fail while in standby and that all three components have the same constant failure rate when on-line. The following states are defined as follows:

\begin{tabular}{|l|l|l|l|}
\hline State & Component 1 & Component 2 & Component 3 \\
\hline 1 & On-line & Standby & Standby \\
\hline 2 & Failed & On-line & Standby \\
\hline 3 & Failed & Failed & On-line \\
\hline 4 & Failed & Failed & Failed \\
\hline
\end{tabular}

This leads to following differential equations:

$$
\begin{aligned}
& \frac{d p_{1}(t)}{d t}=-\lambda P_{1}(t) \\
& \frac{d p_{2}(t)}{d t}=\lambda P_{1}(t)-\lambda P_{2}(t) \\
& \frac{d p_{3}(t)}{d t}=\lambda P_{2}(t)-\lambda P_{3}(t)
\end{aligned}
$$

with initial conditions $\mathrm{P}_{1}(0)=1, \mathrm{P}_{2}(0)=0$, and $\mathrm{P}_{3}(0)=0$. The solution is as follows:

$$
\begin{aligned}
& P_{1}(t)=e^{-\lambda t} \\
& P_{2}(t)=\lambda t e^{-\lambda t} \\
& P_{3}(t)=\frac{\lambda^{2} t^{2}}{2} e^{-\lambda t}
\end{aligned}
$$

Since the system is functioning while in any of the first three states,

$$
R(t)=e^{-\lambda t}\left[1+\lambda t+\frac{\lambda^{2} t^{2}}{2}\right]
$$

and,

$$
\mathrm{MTTF}=3 / \lambda
$$




\subsubsection{Degraded Systems}

Some systems may continue to operate in a degraded mode following certain types of failures. The system may continue to perform its function but not at a specified operating level. For example, a computer system may not be able to access all of its direct access storage devices, a copying machine may not be able to automatically feed originals and may thereby require manual operation, or a multi-engine aircraft may experience a problem in one of its engines [8]. Whether the degraded mode is considered a failure or not must be determined as part of the reliability specification. However, if it is important to distinguish the degraded state from that of a complete failure, then Markov analysis can be utilized if constant failure rates are assumed.

Defining the states of a system as fully operational (state 1), degraded (state 2), and failed (state 3), the RTD is as shown in Figure 5.10.

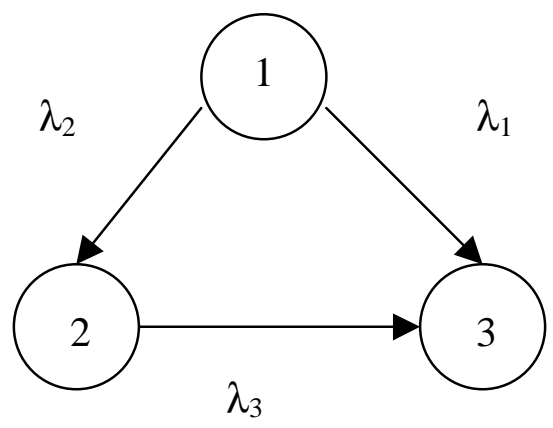

Figure 5.10: Reliability Transition Diagram for Degraded Systems

The differential equations are

$\frac{d P_{1}(t)}{d t}=-\left(\lambda_{1}+\lambda_{2}\right) P_{1}(t)$

$\frac{d P_{2}(t)}{d t}=\lambda_{2} P_{1}(t)-\lambda_{3} P_{2}(t)$

The solution to Eq. (5.40) and (5.41) is given by

$$
\begin{aligned}
& P_{1}(t)=e^{-\left(\lambda_{1}+\lambda_{2}\right) t} \\
& P_{2}(t)=\frac{\lambda_{2}}{\lambda_{1}+\lambda_{2}-\lambda_{3}}\left[e^{-\lambda_{3} t}-e^{-\left(\lambda_{1}+\lambda_{2}\right) t}\right]
\end{aligned}
$$

Finally, P3(t) $=1-\mathrm{P} 1(\mathrm{t})-\mathrm{P} 2(\mathrm{t})$ 
And,

$M T T F=\frac{1}{\lambda_{1}+\lambda_{2}}+\frac{\lambda_{2}}{\lambda_{1}+\lambda_{2}-\lambda_{3}}\left[\frac{1}{\lambda_{3}}-\frac{1}{\lambda_{1}+\lambda_{2}}\right]$

\subsection{State Independent System Reliability - Tie Set and Cut Set} Algorithm

The term cut set means a minimal set of system components which when fail, cause the system to fail. This technique consists of the following three distinct steps [21].

1. Find minimal paths or tie sets.

2. Find cut sets using the minimal paths found in step 1 .

3. Determine the system reliability using cut sets found in step 2 .

\subsubsection{Tie Set or Minimal Paths Algorithm}

A tie set is a minimal set of components from start to end node. Failing of all tiesets causes the system to fail, or if components in any of the tie set works, the system works. A minimal path tree shows the link of all components from the end to the start node. The minimal paths can be obtained by tracing network end to start node.

\section{Assumptions:}

1. A network represents the actual system.

2. Two or more parallel branches connected between any pair of nodes are merged to form a single-branch.

3. User identifies previous components for each component in the system.

\section{Algorithm:}

1. Place end node on top of the tree (level 1).

2. Check for previous matrix for predecessor of end node. Put them at level 2.

3. For each component at a given level which is not start node, do the following:

3.1 Obtain predecessor for a given component.

3.2 For predecessor connected to given component check the following:

3.2.1 Is the component connected the same way somewhere on the minimal path traced out so far from end to present component? If yes, go to step 3.2 for next component (repetition). 


\subsubsection{If not place them in level 3 and go to step 3 .}

If start node, then go to step 4 - backward procedure.

4. Go back on one level and check for other predecessor. Go to step 3. Repeat until all predecessors are taken into account at all levels.

After obtaining all paths of network one can eliminate redundant paths to form minimal paths. This can be done by comparing the current component at the current level with the components from the previous level in the corresponding branch.

\subsubsection{Determination of Cut sets from given Minimal Paths}

Determination of cut sets is important for evaluation of system reliability. Evaluation of the combination of component failures that discontinue the minimal paths can obtain minimal cut sets. Boolean logic is used to obtain cut sets from the matrix containing the minimal paths.

\section{Assumptions:}

1. Minimal paths are represented as binary matrix $T_{\mathrm{MxN}}$, where $\mathrm{M}$ is the number of minimal paths and $\mathrm{N}$ is the number of components. $T[i, j]=1$, if component $\mathrm{j}$ is in cut set $\mathrm{i}, 0$ otherwise. $\mathrm{T}_{\mathrm{j}}$ is $\mathrm{j}^{\text {th }}$ column vector of $\mathrm{T}_{\mathrm{MxN}}$ matrix.

\section{Algorithm:}

First order cut sets:

For all $\mathrm{j}^{\text {th }}$ column vector of $\mathrm{T}_{\mathrm{MxN}}$ matrix do:

1. If $T_{j i}=1$ for all $i=1,2 \ldots . M$, the corresponding element $\mathrm{j}$ is a single element cut. No single-elements cuts otherwise.

Second order cut sets:

For all $\mathrm{j} \neq \mathrm{c}$ (column vectors) of $\mathrm{T}_{\mathrm{MxN}}$ matrix:

2. If $\mathrm{T}_{\mathrm{ji}}+\mathrm{T}_{\mathrm{ci}}=1$ for all $\mathrm{i}=1,2 \ldots \mathrm{M}$, then the elements $\mathrm{j}$ and $\mathrm{c}$ form a second order cut set. Here, ' + ' indicates the logic sum or union. Eliminate non-minimal cut set as follows:

2.1 After obtaining a cut set, check it against all cuts of order one.

2.1.1 If the cut set contains a smaller order cut set, eliminate it.

2.1.2 Proceed to next cut set (step 2) until all cut sets are identified.

3. Repeat the procedure for all possible cuts of higher order $1,2 \ldots . . \mathrm{N}$, where $\mathrm{N}$ is the number of elements in the system.. 


\subsubsection{Example}

Let there be a system that has 5 components, $X_{1}$ through $X_{5}$. Connection as shown in Figure 5.11. Start and end are pseudo nodes.

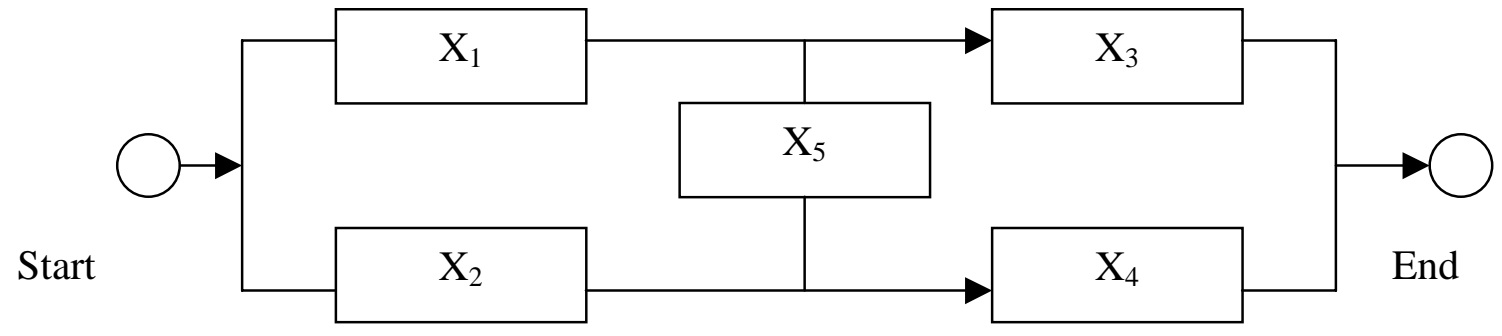

Figure 5.11: Complex System

The previous component $(\mathrm{PC})$ matrix of this system is given as:

\begin{tabular}{|c|c|c|c|c|c|c|}
\hline & & $\mathbf{X}_{1}$ & $\mathbf{X}_{2}$ & $\mathbf{X}_{3}$ & $\mathbf{X}_{4}$ & $X_{5}$ \\
\hline & $\overline{\mathbf{X}_{1}}$ & 0 & 0 & 0 & 0 & 0 \\
\hline & $\mathbf{X}_{2}$ & 0 & 0 & 0 & 0 & 0 \\
\hline $\mathrm{PC}=$ & $\mathbf{X}_{3}$ & 1 & 0 & 0 & 0 & 1 \\
\hline & $\mathbf{X}_{4}$ & 0 & 1 & 0 & 0 & 1 \\
\hline & $\mathbf{X}_{5}$ & 1 & 1 & 0 & 0 & 0 \\
\hline
\end{tabular}

Rows (i) and columns (j) of the PC matrix correspond to component number. $\mathrm{PC}_{\mathrm{ij}}$ $=1$ if component $\mathrm{j}$ is previous to component $\mathrm{i}$. To form the minimal path tree the End $(\mathrm{E})$ node is placed at the level 1. All nodes that are predecessors to the End node are placed at the level 2. Process is repeated until no previous nodes exist. The tree for network is shown in Figure 5.12.

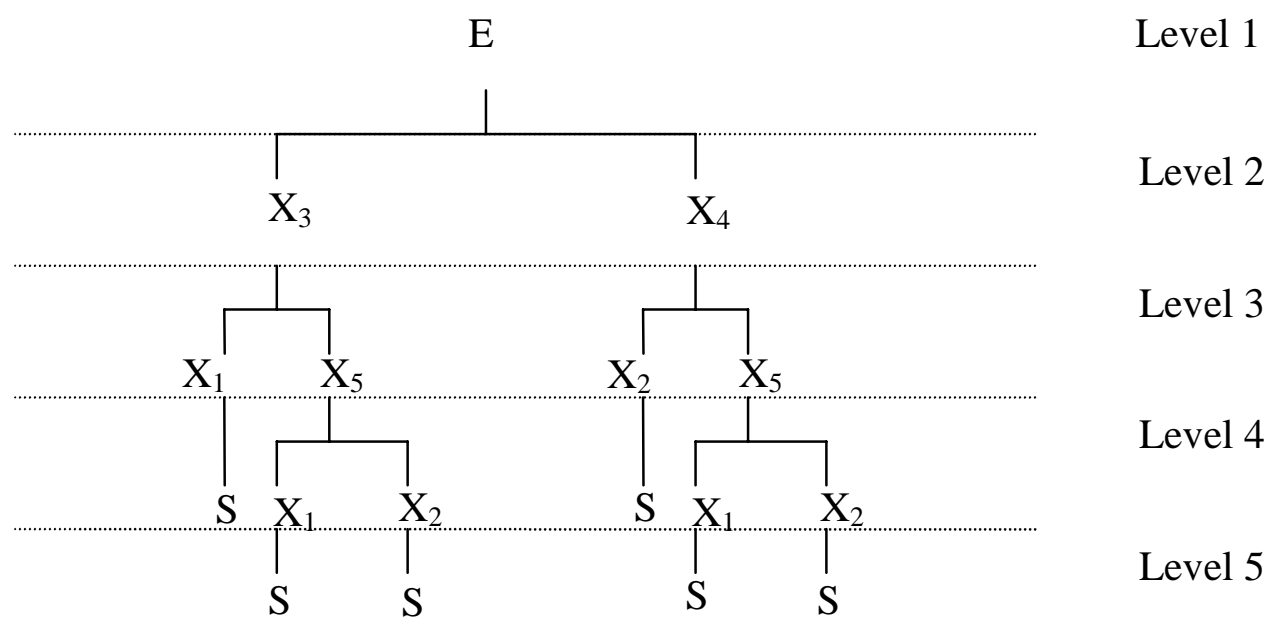

Figure 5.12: Tree Structure of the Complex System 
The minimal path (MP) matrix is generated by the above procedure. From MP matrix the End (E) and the Start (S) nodes can be eliminated resulting in the tie set, T.

$$
\mathrm{MP}=\left[\begin{array}{lllll}
\mathrm{E} & \mathrm{X}_{3} & \mathrm{X}_{1} & \mathrm{~S} \\
\mathrm{E} & \mathrm{X}_{3} & \mathrm{X}_{5} & \mathrm{X}_{2} & \mathrm{~S} \\
\mathrm{E} & \mathrm{X}_{4} & \mathrm{X}_{2} & \mathrm{~S} & \\
\mathrm{E} & \mathrm{X}_{4} & \mathrm{X}_{5} & \mathrm{X}_{1} & \mathrm{~S} \\
\mathrm{E} & \mathrm{X}_{4} & \mathrm{X}_{5} & \mathrm{X}_{2} & \mathrm{~S} \\
\mathrm{E} & \mathrm{X}_{3} & \mathrm{X}_{5} & \mathrm{X}_{1} & \mathrm{~S}
\end{array}\right] \quad \mathrm{T}=\left[\begin{array}{cccc}
\mathrm{X}_{3} & \mathrm{X}_{1} & \\
\mathrm{X}_{3} & \mathrm{X}_{5} & \mathrm{X}_{2} \\
\mathrm{X}_{4} & \mathrm{X}_{2} & \\
\mathrm{X}_{4} & \mathrm{X}_{5} & \mathrm{X}_{1} \\
\mathrm{X}_{4} & \mathrm{X}_{5} & \mathrm{X}_{2} \\
\mathrm{X}_{3} & \mathrm{X}_{5} & \mathrm{X}_{1}
\end{array}\right]
$$

\subsubsection{Algorithm for Calculating System Reliability}

This section describes a computer algorithm to determine the reliability of a complex system. The algorithm is based on [21]. The algorithm uses a sequence of prediction equations, which provides increasingly closer bounds on the system reliability. It is based on the knowledge of tie set and cut set. Let $T_{m}$ is the $m^{\text {th }}$ tie set, $M$ (number of tiesets) in number and $\mathrm{C}_{\mathrm{k}}$ is $\mathrm{k}^{\text {th }}$ cut set, $\mathrm{K}$ (number of cutsets) in number. Then system reliability is given by $\mathrm{R}_{\mathrm{s}}=\mathrm{P}\left\{\mathrm{T}_{1} * \mathrm{~T}_{2} \ldots \ldots * \mathrm{~T}_{\mathrm{M}}\right\}=\mathrm{P}$ (at least one tie set is good).

Expressed in terms of cut sets, $\mathrm{R}_{\mathrm{s}}=\mathrm{P}\left\{\mathrm{C}_{1}+\mathrm{C}_{2}+\ldots .+\mathrm{C}_{\mathrm{k}}\right\}=\mathrm{P}$ (cut sets are good, i.e. contain at least one element of the set which is operative). Equivalently, the system failure is expressed as:

$$
\begin{aligned}
& 1-\mathrm{R}_{\mathrm{s}}=\mathrm{P}\left\{\mathrm{T}_{1}^{\prime}{ }_{1} \mathrm{~T}_{2}{ }_{2} * \ldots \ldots * \mathrm{~T}^{\prime}{ }_{\mathrm{M}}\right\}=\mathrm{P}\{\text { all tie sets have a failure }\} \text { or } \\
& 1-\mathrm{R}_{\mathrm{s}}=\mathrm{P}\left\{\mathrm{C}_{1}^{\prime}+\mathrm{C}_{2}^{\prime}+\ldots \ldots .+\mathrm{C}_{\mathrm{K}}^{\prime}\right\}=\mathrm{P}\{\text { all tie sets have a failure }\} \text { or }
\end{aligned}
$$

Where $T^{\prime}{ }_{M}$ and $C^{\prime}{ }_{K}$ are the compliments of the events $T_{M}$ and $C_{K}$ respectively. From the above reliability expression bounds can be obtained by using the basic probabilistic inequalities.

$$
\begin{aligned}
& \mathrm{R}_{\mathrm{s}}=\mathrm{P}\left\{\mathrm{T}_{1}+\mathrm{T}_{2}+\ldots . .+\mathrm{T}_{\mathrm{M}}\right\} \leq \sum_{i=1}^{M} \mathrm{P}\left\{\mathrm{T}_{\mathrm{i}}\right\} \\
& \mathrm{R}_{\mathrm{s}}=\mathrm{P}\left\{\mathrm{T}_{1}+\mathrm{T}_{2}+\ldots . .+\mathrm{T}_{\mathrm{M}}\right\} \geq \sum_{i=1}^{M} \mathrm{P}\left\{\mathrm{T}_{\mathrm{i}}\right\}-\sum_{i=1}^{M-1} \sum_{j=i+1}^{M} P\left\{T_{i} * T_{j}\right\}, \quad 1 \leq i, j \leq M
\end{aligned}
$$


The upper and lower bounds $\mathrm{R}_{\mathrm{U} 1}$ and $\mathrm{R}_{\mathrm{L} 1}$ to the reliability are given by:

$$
\begin{gathered}
\mathrm{R}_{\mathrm{U} 1}=\sum_{i=1}^{M} \mathrm{P}\left\{\mathrm{T}_{\mathrm{i}}\right\} \\
\mathrm{R}_{\mathrm{L} 1}=\sum_{i=1}^{M} \mathrm{P}\left\{\mathrm{T}_{\mathrm{i}}\right\}-\sum_{i=1}^{M-1} \sum_{j=i+1}^{M} P\left\{T_{i} * T_{j}\right\},
\end{gathered}
$$

In the same manner, another upper bound is obtained

$$
\mathrm{R}_{\mathrm{U} 2}=\sum_{i=1}^{M} \mathrm{P}\left\{\mathrm{T}_{\mathrm{i}}\right\}-\sum_{i=1}^{M-1} \sum_{j=i+1}^{M} P\left\{T_{i} * T_{j}\right\}+\sum_{i=1}^{M-2} \sum_{j=i+1 k=j+1}^{M-1} \sum^{M} P\left\{T^{*} T_{j} * T_{k}\right\}, \quad 1 \leq i, \quad j, k \leq M
$$

Similarly inequalities can be applied to cut sets to obtain another upper and lower bounds.

$$
\mathrm{R}_{\mathrm{sys}} \geq 1-\sum_{i=1}^{K} \mathrm{P}\left\{\mathrm{C}_{\mathrm{i}}^{\prime}\right\}, \text { thus } \quad R_{L 2}=1-\sum_{i=1}^{K} P\left\{C^{\prime}{ }_{i\}}\right.
$$

$\mathrm{R}_{\mathrm{U} 3}=1-\sum_{i=1}^{K} \mathrm{P}\left\{\mathrm{C}_{\mathrm{i}}^{\prime}\right\}+\sum_{k<m} P\left\{C_{K}^{\prime}{ }_{K}^{*} C_{M}\right\}, \quad 1 \leq K, \quad M \leq K$

Thus by using the equations of reliability based on tie set and cut set series of upper and lower bounds are generated which when converge provides the actual system reliability. The above equations were for three tiesets. If a system has more tiesets, similar equations are obtained. The only difference is that as the tiesets increase, the combination is among more tiesets till the combination of total number of tiesets, with +/signs placed alternatively among them. This when converges, gives the actual reliability of the system. 


\section{Chapter 6: Software Tool for Reliability Estimation (STORE)}

A Software Tool for Reliability Estimation (STORE) is described in this chapter. It was developed to facilitate the calculation of reliability of a component or a system using the different hardware/software models and system reliability algorithm discussed in chapters 3, 4 and 5. Apart from calculating the reliability of a component or a system, it also provides useful statistical analysis, which helps a user to select the model for given data. STORE is capable of the following,

- Study existing statistical models to estimate reliability of Hardware-Software components.

- Study existing algorithm to compute reliability of complex systems.

- Implement an algorithm to compute tiesets and cutsets of complex systems.

- Compute reliability (with known or unknown parameters) of state dependent and state independent systems.

\subsection{STORE Description}

STORE is a Windows based reliability estimation tool. It is implemented using Microsoft Visual Basic 6 [33]. As a Microsoft's premium programming language, Visual Basic 6 is the easiest object-oriented programming approach for application development. It has a sophisticated front end, with powerful windows features and the backend, where the logic is developed that interacts with the front end. The Jet Database Engine was used to establish such connection[33]. STORE essentially consists of forms or screens. These forms are similar to those displayed by Windows 95/98/NT. There are control buttons or toolbox controls, which are used on the custom designed forms. These control buttons generally build the interface between the program and the user. The major tool used in this program consists of flexigrid, which allows user to open data in tabular form from a data file. A file open menu, which allows the user to select data files from the directory in their PC or disk and open the data in the program.

STORE is intended for practicing reliability engineers, who are interested in calculating and analyzing reliability of hardware and software components and systems. It allows a user to analyze hardware, software and system reliability under one 
environment. STORE also has capability to calculate reliability and Mean Time To Failure (MTTF) for state dependent systems, which most of the commercial reliability tools do not support.

\subsection{STORE Menu Structure}

Various forms constitute the building block of STORE. These forms and associated code allow the user to input data and estimate the reliability. The forms are discussed here as they appear in the program and the code is shown in Appendix V. Figure 6.1 shows the menu structure of STORE.

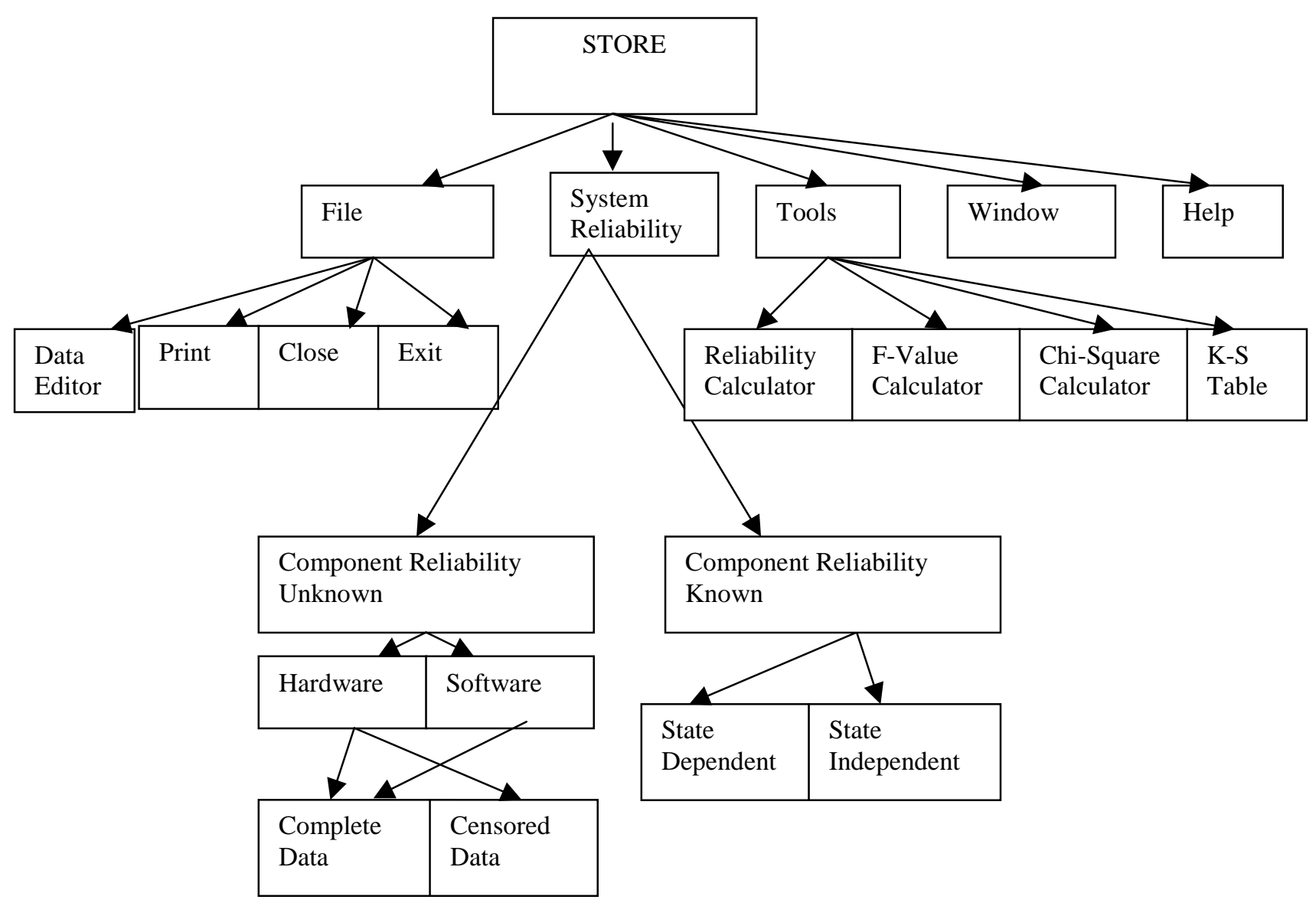

Figure 6.1: Menu Structure 


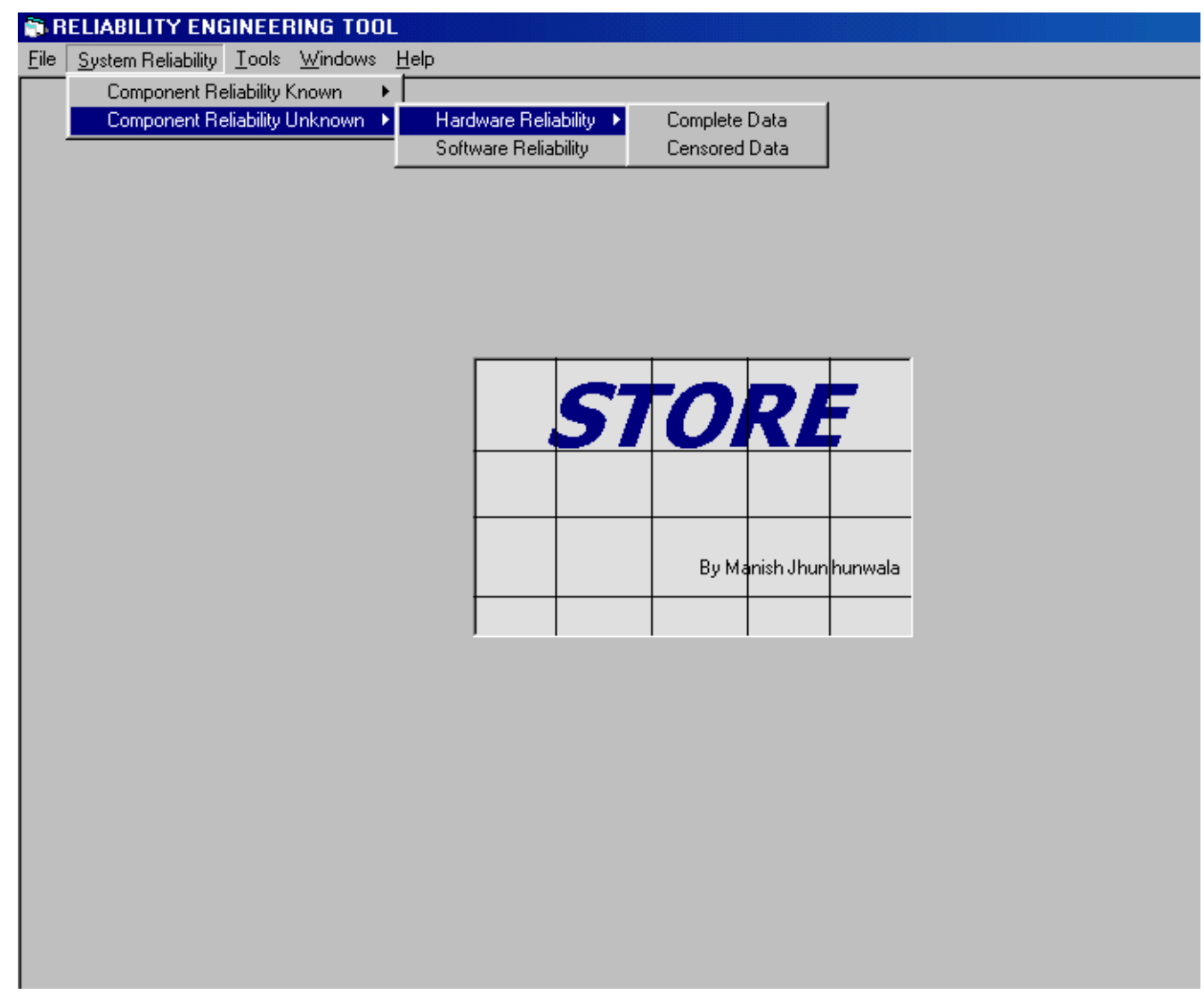

Figure 6.2: Opening Screen

When the software is started the screen as shown in Figure 6.2 is displayed. The options on this screen are File, System Reliability, Tools, Windows and Help.

Under the File menu, there is Data Editor, which allows the user to create data file or to edit an existing data file. The "Print" option allows user to print the results obtained. The "Close" option closes the applicatiuon and "Exit" terminates the program.

Under system reliability, there are two submenus, one for system reliability when the component reliability is known and other when component reliability is unknown. When component reliability is known, the user can work upon both state dependent and state independent systems. If the component reliability is unknown, then user can compute component reliability using Hardware or Software Reliability menu. The hardware reliability can be calculated for both complete and censored data, whereas software is only for complete data.

Under the Tools menu, there is F-value calculator, Reliability calculator, Kolmogorv-Smirnov Table and Chi-square calculator. These tools assist the user to select the "best" model. For example, if the user needs to check the distribution for different 
number of risk, this can be done using the tools instead of going through complete program.

The "Windows" menu helps the user arrange the window frames in cascade or tile form. Also this menu lists all the open forms of the program.

The Help menu provides the basic help explaining terms used in reliability, notations and nomenclature used in program and how to use the program.

\subsubsection{Data Editor}

The Data Editor is a text editor for this particular software. The user can enter the failure data for hardware or software item and can save the file with "hrd" for hardware or "sft" for software extension. Also user can enter system block in this editor and save it using "sys" for system as extension. The different extensions allows the user to differentiate between the different types of data files. The data files stored through the data editor are then recalled in the respective application for further evaluation. The sample screen of the data editor is shown in Figure 6.3.

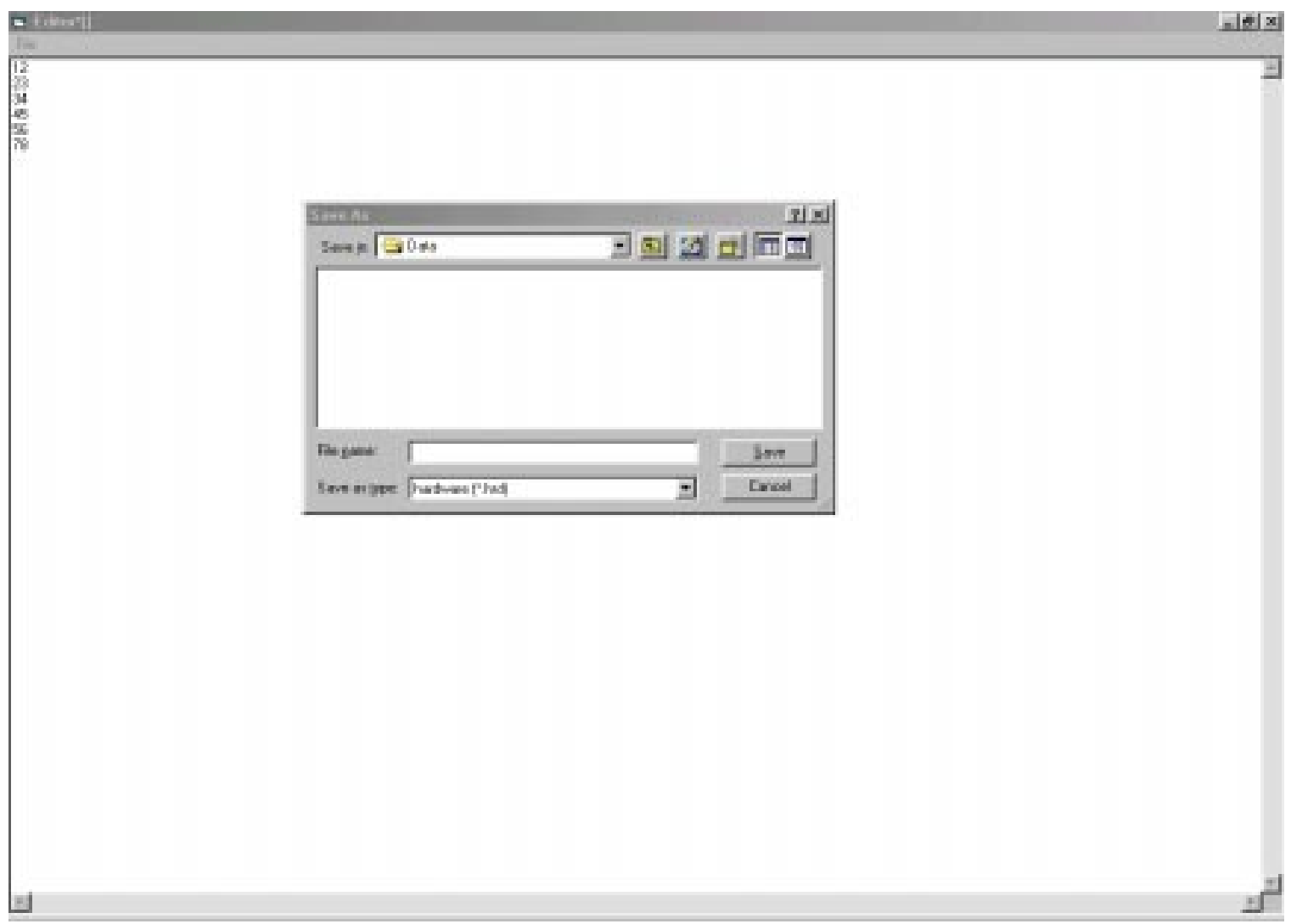

Figure 6.3: Data Editor Screen 


\subsubsection{Hardware Reliability - Complete Data}

This screen opens when the user clicks the "Complete data" under the Hardware reliability option of "Open" submenu. This screen helps the user in specifying information about the failure data. The file control provision allows the user to select the data file for the particular hardware product. When the selected file is open, user can see the data in the data grid. Data grid is only for displaying the data, the user cannot edit the data through data grid. If the user wants to edit the data, the data file has to be opened in the "Data Editor". User can then select the appropriate distribution. This selection is possible through the "option button". One distribution at a time can be selected. On clicking "Hardware Reliability" button, program calculates the parameters for that particular distribution and also the reliability of the product for a specified mission time. The output of the program is displayed in the object "label". User can experiment with different distributions and compare the output. Also user can print the screen by selecting the print option under "File" menu. "Clear" button allows the user to erase all the previously calculated information and start afresh with a data file. The sample screen of hardware reliability is shown in Figure 6.4.

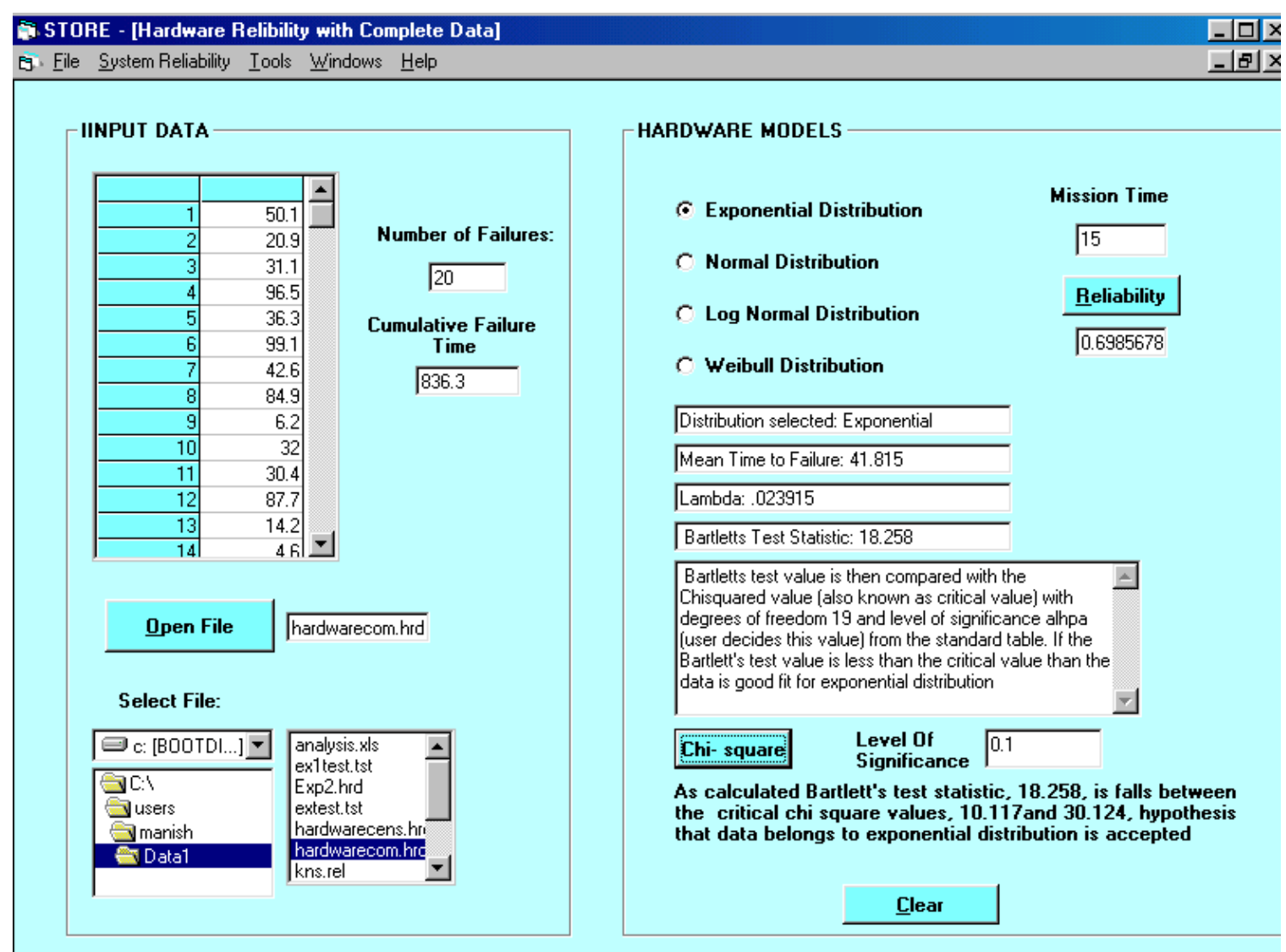

Figure 6.4: Hardware Reliability - Complete data screen 


\subsubsection{Hardware Reliability - Censored Data}

This screen opens when user user clicks censored in hardware reliability option under "Open" submenu. This screen helps the user to open the failure data in the grid on the upper right hand side of the screen. Then the user can compute reliability, do statistical analysis for the censored data of Type I.

The data is displayed in this screen from a file, which can be opened by clicking the "Open" button on the screen and then clicking "Extract". In this screen, the user can select from three different distributions. This selection is possible through the option button shown on the screen. User can select only one option at a time. The user is also required to input potential number of failures, test time and time t. The program calculates number of failures. Once the required information is supplied on the screen, just click the "Reliability" button. This action will calculate reliability, corelation, mean time to failure and test statistic for the item for a specified test time. The output is displayed in the text box, explaining the results. The goodness of fit test is performed by specifying the level of significane in the text box. After specifying a level of significance, the program computes Goodness of Fit test and displays the output in the text box. The sample screen of hardware reliability for censored data is shown in Figure 6.5 .

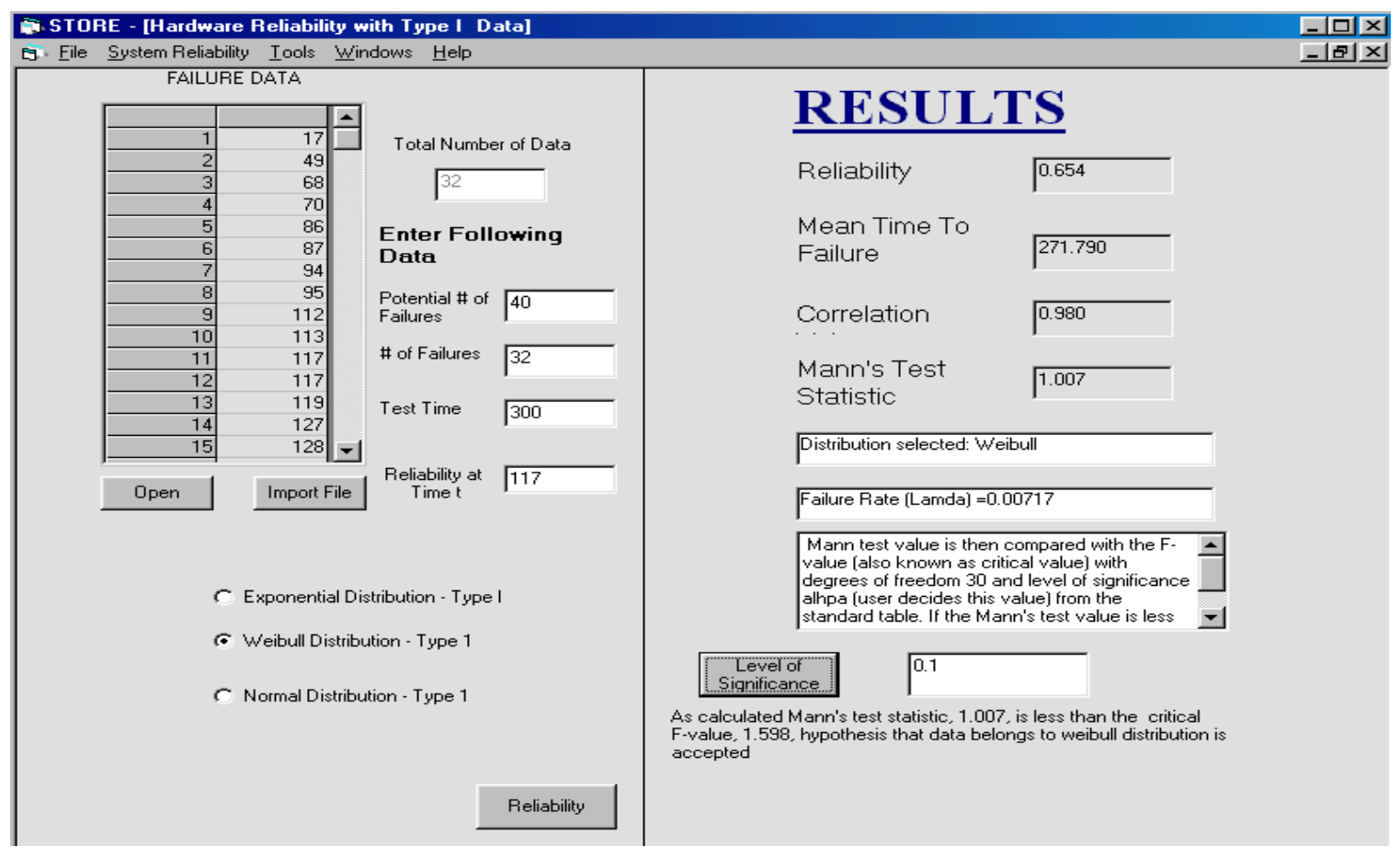




\subsubsection{Software Reliability}

Like hardware reliability, this screen is designed to analyze the software failure data. There is provision for opening the failure data file and selecting the different distributions. By clicking on the "Failure Intensity" button, the program calculates the failure intensity for that particular software failure data. There is also provision for calculating the failure intensity for the user specified time. This helps the user to know how long the software product has to be tested. This screen also displays the graphical behavior of the fitted distribution. This is achieved with the special control "MS-Chart Control". For this the two columns of the data grid are designated as X-axis and $\mathrm{Y}$-axis, and then the graph is plotted between them. The sample screen for software reliability is shown in Figure 6.6.

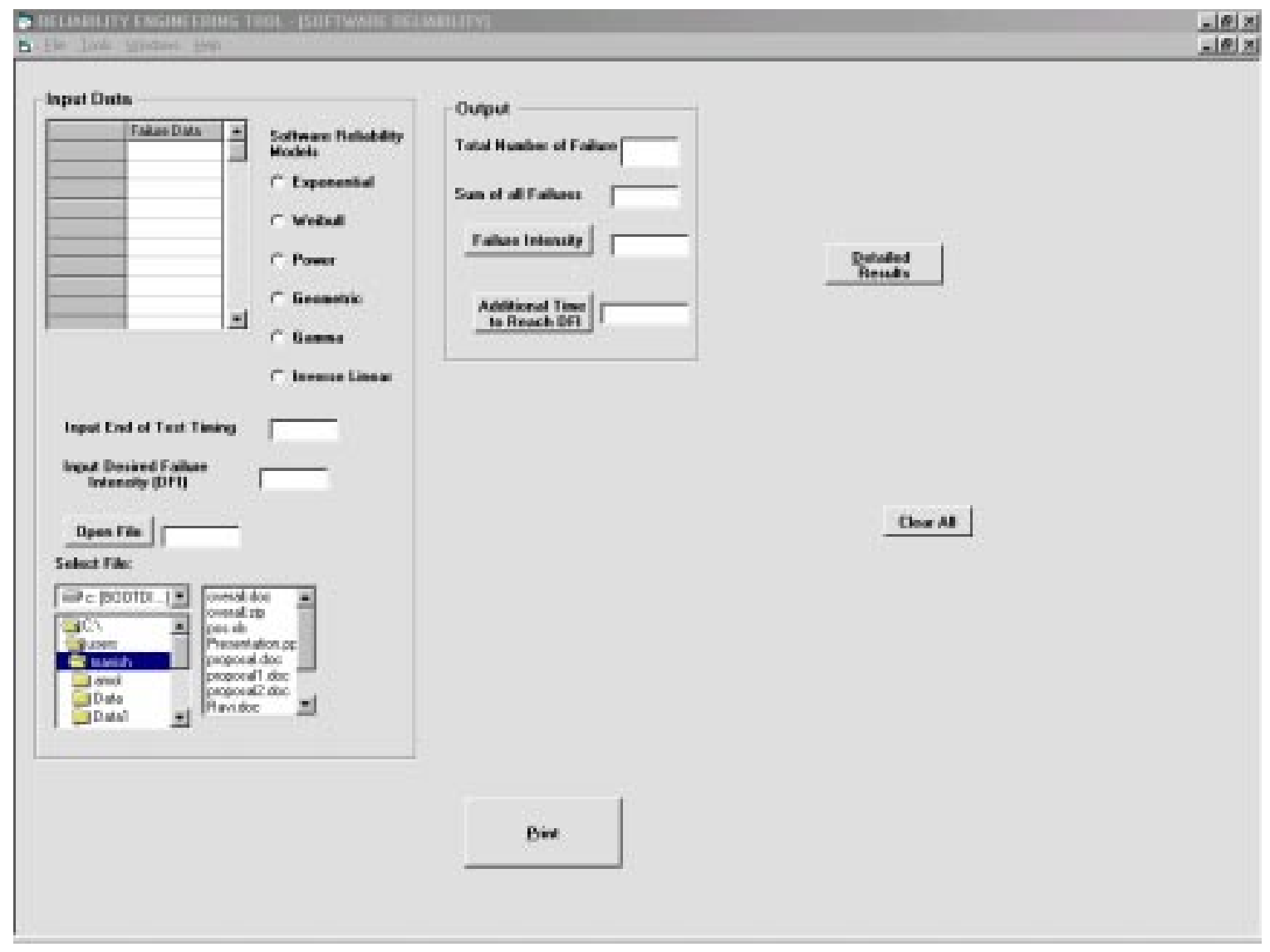

Figure 6.6: Software Reliability Screen

\subsubsection{Result Screen}

This screen is associated with the software reliability screen. It cannot be called from the menu bar. Once the software reliability screen is executed to analyze the failure 
data, the results screen can be called by clicking "Detailed Results" button. On this screen all of the necessary information related to the analyzed data is displayed. Data grid is used to display the failure intensity and the reliability of the software at the end of a particular test time. Also important statistical information pertaining to the data is displayed. By clicking "Graph" the user is able to see various graphs related with the analyzed data as shown in Figure 6.7.

\begin{tabular}{|c|c|c|c|c|c|c|c|}
\hline Eate-16a & Foteress & Founinat & Fentey & def & Silunet & $F r g=n$ & 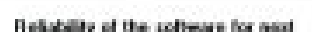 \\
\hline 1 & 2 & acests & 10084 & DEc & 00012 & of & 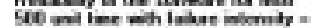 \\
\hline 3 & Is & DDOAI & 13060 & $\operatorname{arcth} 4$ & $000 \pi$ & or ${ }^{-}$ & ละaż is 914 \\
\hline 3 & 185 & docestris & towat & ouses & ock 35 & $0 \mathrm{c}$ & \\
\hline 는 & 227 & gocesn & $1000 \%$ & 00654 & 0 cesia & oc & \\
\hline 라 & $x^{2}$ & acedirs & 100012 & aonix & $000 \mathrm{sen}$ & or & 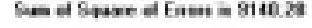 \\
\hline 逢 & उा & acters & 100019 & acras & 00021 & or & \\
\hline 7 & \$5 & acotose & iokst & $\operatorname{ain} 271$ & Doussos & $0 \mathrm{c}$ & \\
\hline 要 & tat & acetsen & Iones & aorat & 00 as 1 & of & \\
\hline 2 & 50 & acetes & $1 \mathrm{axm}$ & acooce & ocence & or & \\
\hline $\overrightarrow{\mathrm{n}}$ & 57 & Dobess & 100050 & $a, x=2$ & $0.004 \pi$ & 01. & \\
\hline ता & 29 & 00000 & lisos & oogent & 00000 & $0 c$ & \\
\hline 녀 & $7 \%$ & 90608 & 10013 & acoser & $00 E \pi$ & or $x$ & Gived \\
\hline 41 & & & & & & \pm & \\
\hline
\end{tabular}
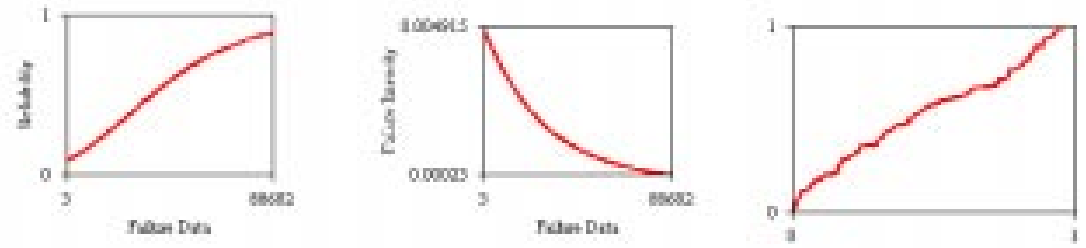

Nelutely tometh Corre

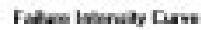

\section{Figure 6.7: Result Screen for Software Reliability}

\subsubsection{System Reliability - State Dependent}

This screen is designed to calculate reliability of two component state depenedent systems. This feature is not available in any commercial reliability tools. This screen is different from the other STORE screens. In this screen there are five tabs that allow user to open desired screen depending upon the state of the system.

Figure 6.8 shows a sample form for such calculations. Different conditions that are used are as follows:

1. Series and Parallel System 
2. Load Sharing System

3. Standby Systems

4. Standby systems with switching failures

5. Three component system.

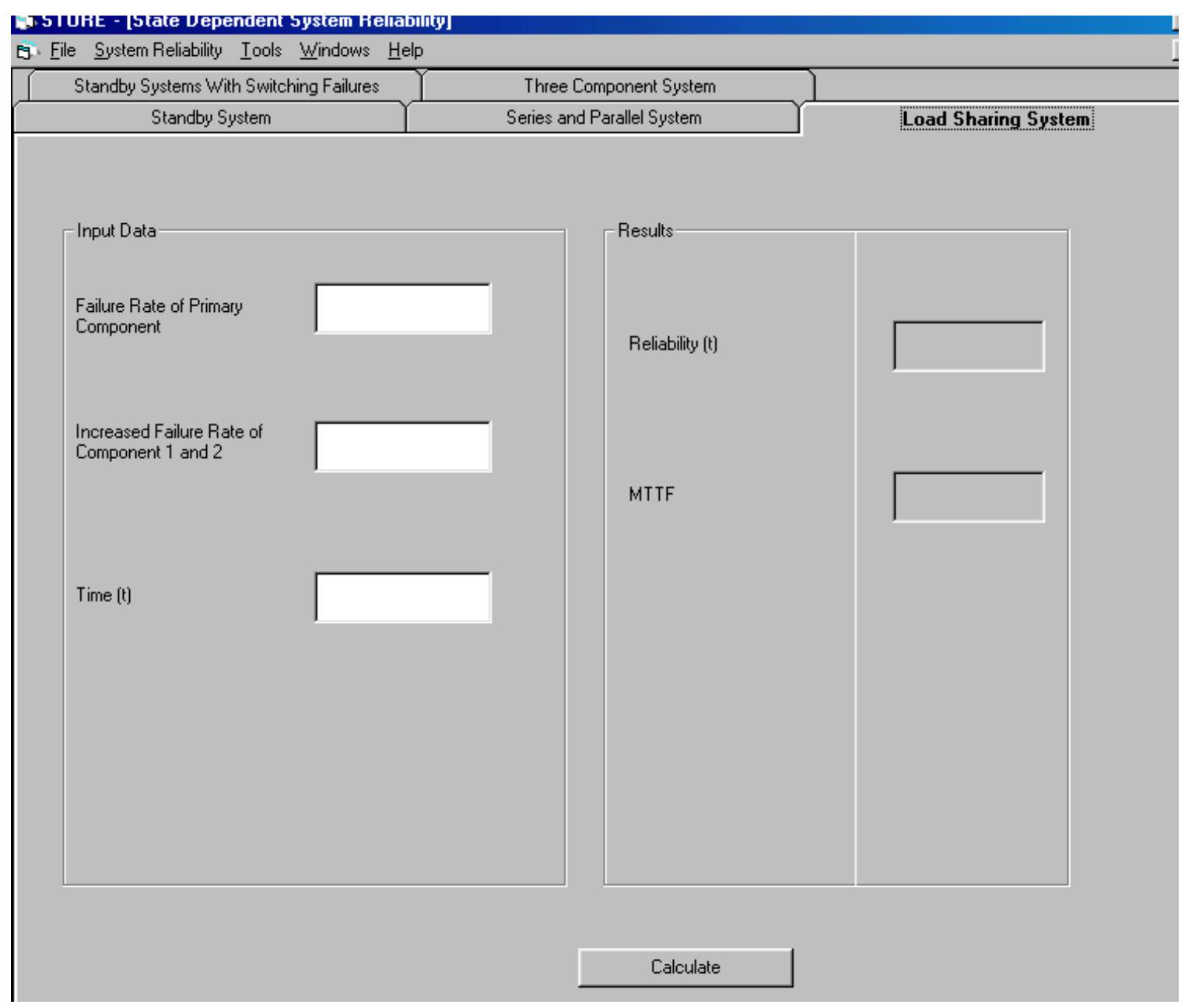

Figure 6.8: State Dependent System Reliability Screen

In this form the user is asked to provide the failure rate and time in state 1 , on the basis of which the program calculates system reliability and MTTF.

\subsubsection{System Reliability}

This screen was designed to calculate system reliability from the given reliability block diagram. On this screen as shown in Figure 6.9, user is required to open the data 
file having the description of the reliability block diagrams along with their reliability values. The file format is explained in Appendix IV. The data file is then displayed on the screen. Once the file is open, the user presses calculate button, which activates the program and the software calculates the tie sets and system reliability. The results are displayed on the screen on pressing "See Results" button. There is a delay (due to computation) to calculate reliability for the system having more than 15 tie sets.

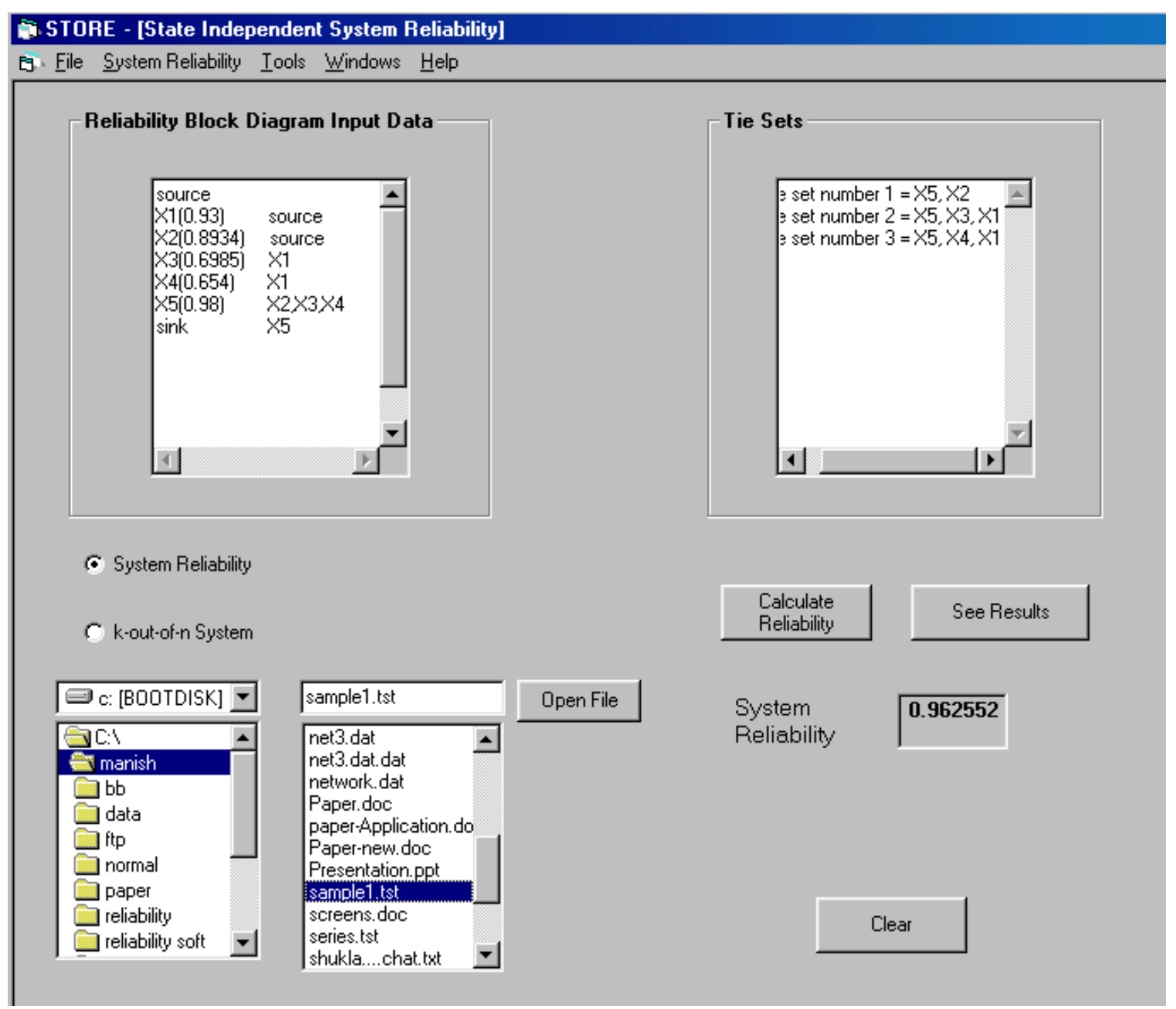

Figure 6.9: System Reliability Screen

\subsubsection{Reliability Calculator}

This screen was designed to calculate the hardware reliability of a component. It can be found under the menu "Tools". This feature is helpful to the user, if user knows the parameters. Here the user can input the parameter for a particular distribution. STORE then calculates the reliability for different mission times using the same failure 
data. Four different distributions are available on this calculator as shown in Figure 6.10 .

\section{RELIABILITY ENGINEERING TOOL - [Hardware Reliability Calculator]}

[]. File System Reliability Iools Windows Help
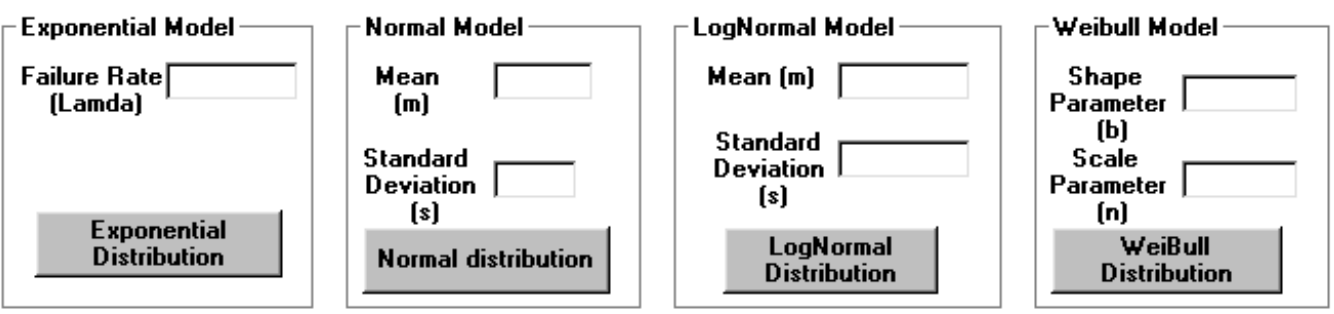

Mission Time

Figure 6.10: Reliability Calculator 


\section{Chapter 7: STORE Application}

This chapter presents the application of STORE to Series system, Parallel system, Series-Parallel system, k-out-of-n systems and complex systems with hardware components, software components and hardware-software components. STORE is also applied to State dependent systems, i.e. Load Sharing system, Standby system, Standby systems with switching failures and degraded systems.

\subsection{Example 1: Series System}

Consider a series system that consists of three components and the reliability values of components $\mathrm{X}_{1}, \mathrm{X}_{2}$ and $\mathrm{X}_{3}$ are $0.9,0.8$ and 0.75 respectively. Estimate reliability of the system [9]. The estimated system reliability value calculated by STORE is 0.54 as shown in Figure 7.2, which is the same as in [9].

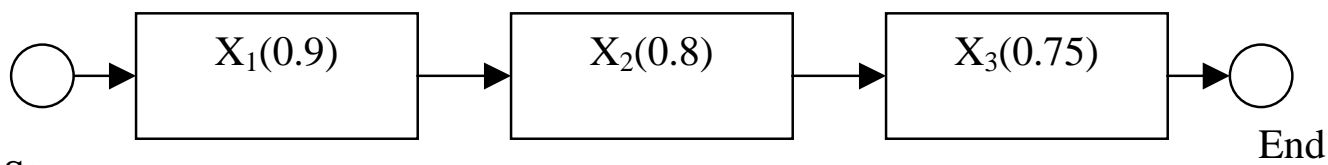

Start

End

Figure 7.1: RBD of Series System

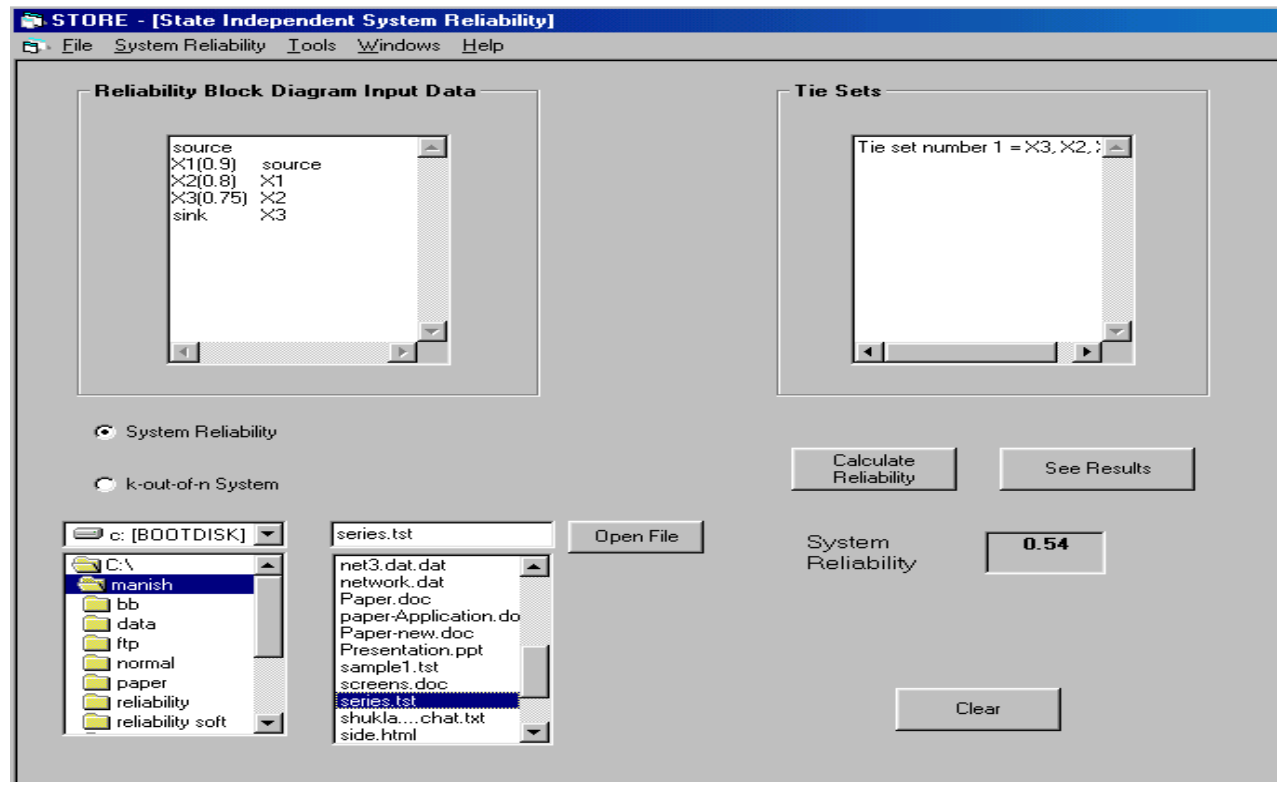

Figure 7.2: Sample STORE Screen for System Reliability of Series System 


\subsection{Example 2: Parallel System}

Consider a parallel system that consists of three components and the reliability values of that components $\mathrm{X}_{1}, \mathrm{X}_{2}$ and $\mathrm{X}_{3}$ are $0.9,0.8$ and 0.75 respectively. Estimate reliability of the system [9]. The estimated system reliability value calculated by STORE is 0.995 as shown in Figure 7.4, which is the same as in [9].

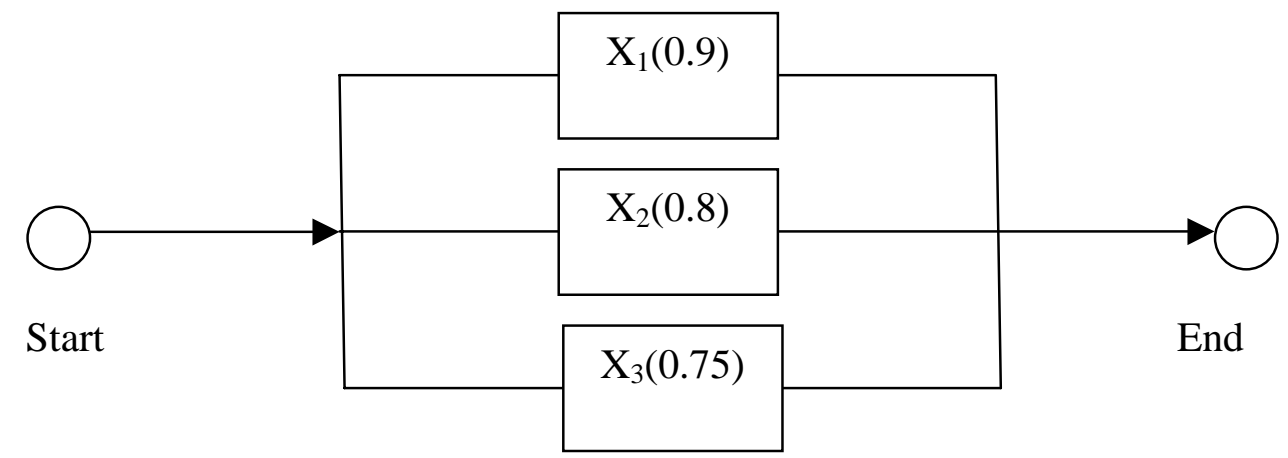

Figure 7.3: RBD for Parallel System

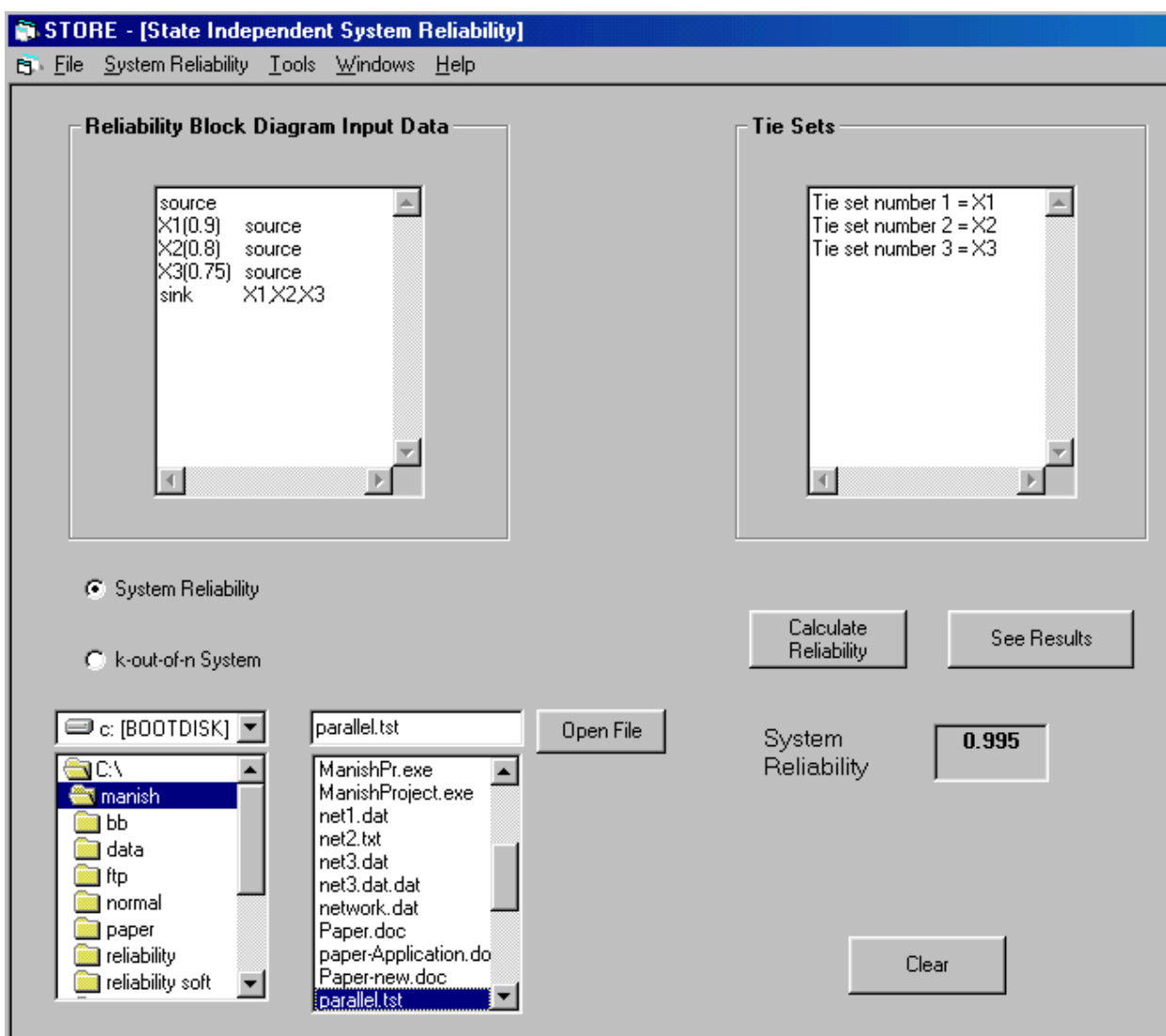

Figure 7.4: Sample STORE Screen for System Reliability of Parallel System 


\subsection{Example 3: k-out-of-n Systems}

Consider a telecommunication system that consists of four different parallel channels. A system is considered operational if any three channels are operational. Determine reliability of the system if reliability value of each channel is 0.85 [9]. The estimated system reliability value calculated by STORE is 0.89048 as shown in Figure 7.6, which is same as in [9].

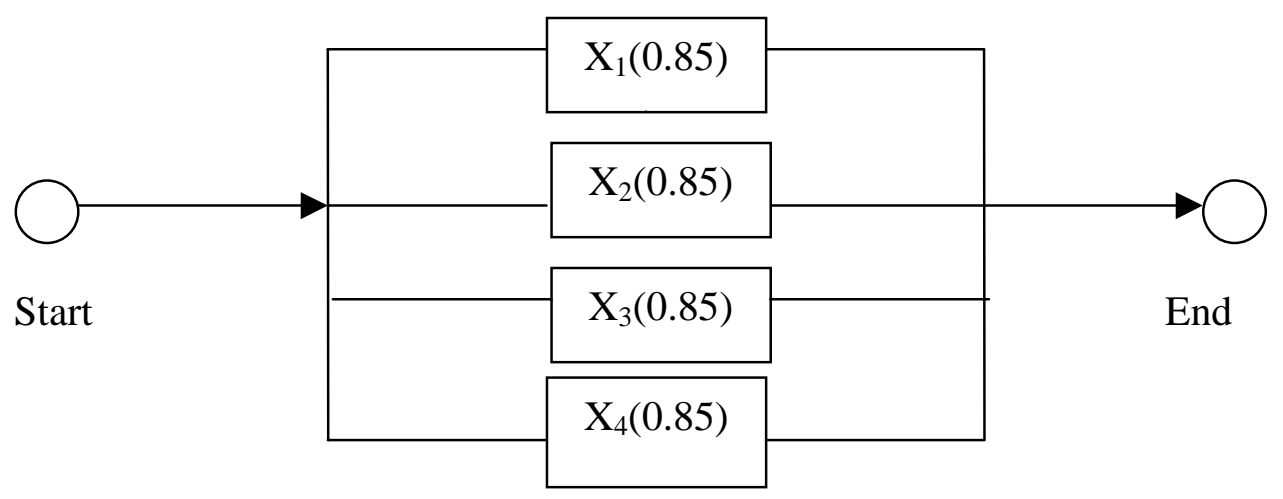

Figure 7.5: RBD for k-out-of-n System

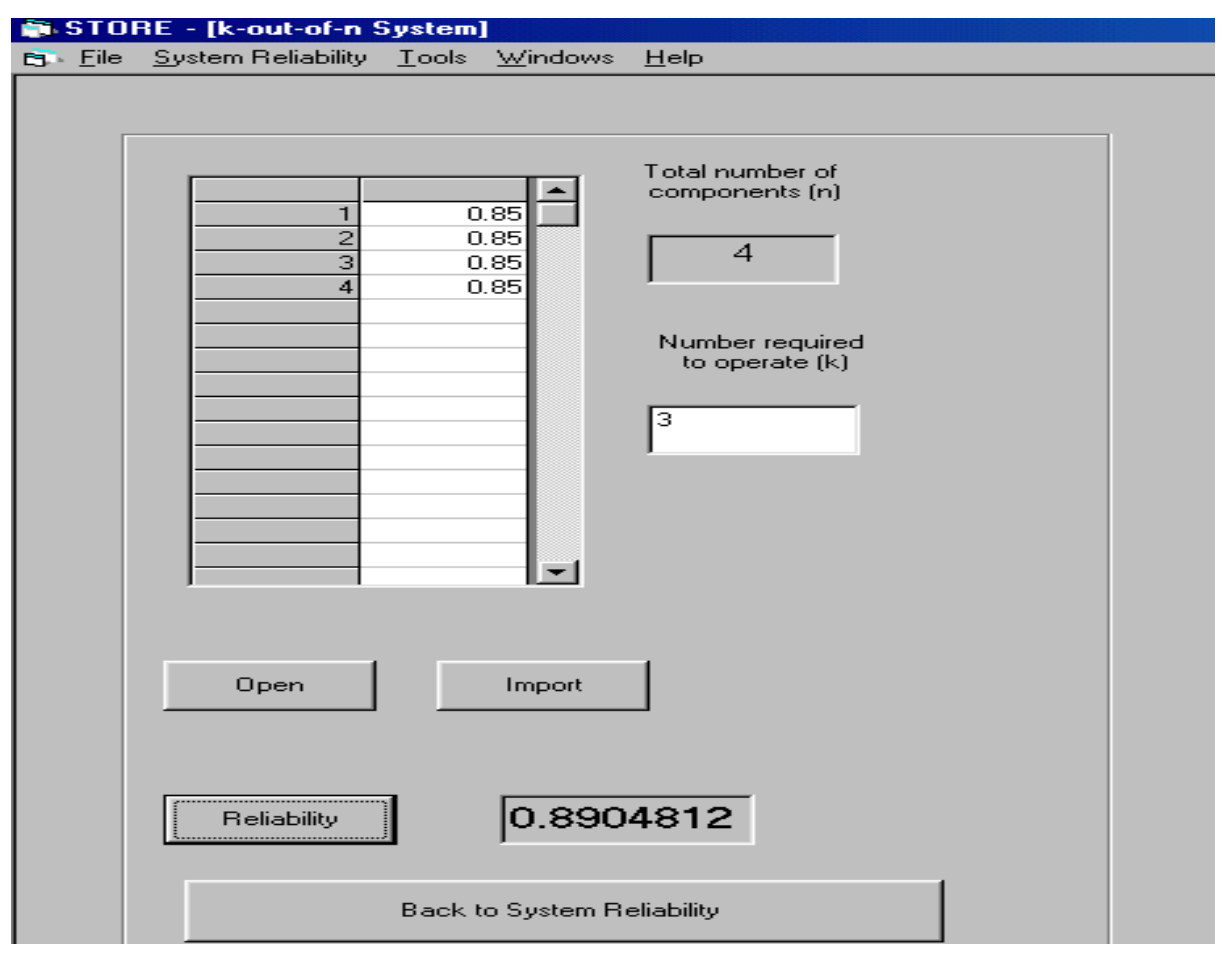

Figure 7.6: Sample STORE Screen for System Reliability of k-out-of-n System 7.4 Example 4: Reliability Block Diagram

Consider a software-hardware combined system as shown in Figure 7.7. 
Assuming that component $\mathrm{X}_{2}$ is software component and rest are hardware components. In order to find reliability of the system, we need to first determine reliability of each component. If the user knows the reliability values of all the components, then STORE can calculates system reliability using tieset algorithm for state independent systems. But if the user does not know reliability of any particular component, then the software asks for the type of the component, i.e. hardware or software. Then depending on the type of the component, the user can use STORE to calculate component reliability based on the failure data. STORE allows the user to select different distributions and determine the best fit.

For example, in our reliability block diagram, the user does not know the reliability of components $\mathrm{X}_{2}, \mathrm{X}_{3}$ and $\mathrm{X}_{4}$.

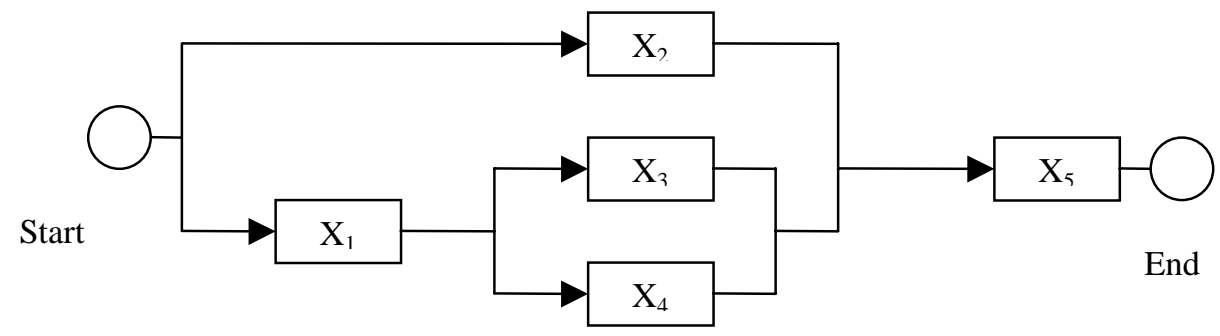

Figure 7.7: Reliability Block Diagram for a Hardware - Software System

\subsubsection{Reliability Calculation for Software Component}

Let us consider that the data in Table 7.1 is the failure data for a particular software component. The different models can be applied to this data. The data was obtained from [16]. It shows software failures experienced at $t_{1}, t_{2} \ldots t_{m}$, for total of number of failures (TNF), time is recorded in CPU seconds. The time at end of observation is given by TET, which in this example does not correspond with the last failure, that is, time at end of test (TET), which occurred at time 91208.

Table 7.1: Software Failure Data

\begin{tabular}{|r|r|r|r|r|r|r|r|r|r|r|r|}
\hline 3 & 33 & 146 & 227 & 342 & 351 & 353 & 444 & 556 & 571 & 709 & 759 \\
\hline 836 & 860 & 968 & 1056 & 1726 & 1846 & 1872 & 1986 & 2311 & 2366 & 2608 & 2676 \\
\hline 3098 & 3278 & 3288 & 4434 & 5034 & 5049 & 5085 & 5089 & 5089 & 5097 & 5324 & 5389 \\
\hline 5565 & 5623 & 6080 & 6380 & 6477 & 6740 & 7192 & 7447 & 7644 & 7837 & 7843 & 7922 \\
\hline 8738 & 10089 & 10237 & 10258 & 10491 & 10625 & 10982 & 11175 & 11411 & 11442 & 11811 & 12559 \\
\hline 12559 & 12791 & 13121 & 13486 & 14708 & 15251 & 15261 & 15277 & 15806 & 16185 & 16229 & 16358 \\
\hline 17168 & 17458 & 17758 & 18287 & 18568 & 18728 & 19556 & 20567 & 21012 & 21308 & 23063 & 24127 \\
\hline 25910 & 26770 & 27753 & 28460 & 28493 & 29361 & 30085 & 32408 & 35338 & 36799 & 37642 & 37654 \\
\hline 37915 & 39715 & 40508 & 42015 & 42045 & 42188 & 42296 & 42296 & 45406 & 46653 & 47596 & 48296 \\
\hline
\end{tabular}




\begin{tabular}{|l|l|l|l|l|l|l|l|l|l|l|l|}
\hline 49171 & 49416 & 50145 & 52042 & 52489 & 52875 & 53321 & 53443 & 54433 & 55381 & 56463 & 56485 \\
\hline 56560 & 57042 & 62551 & 62651 & 62661 & 63732 & 64103 & 64893 & 71043 & 74364 & 75409 & 76057 \\
\hline 81542 & 82702 & 84566 & 88682 & & & & & & & & \\
\hline
\end{tabular}

Table 7.2:Software Reliability Models

\begin{tabular}{|l|l|l|}
\hline \multicolumn{1}{|c|}{ Model Name } & \multicolumn{1}{|c|}{ Failure Intensity Function } & \multicolumn{1}{c|}{ Mean value function } \\
\hline Weibull Distribution & $\lambda(\tau)=b 0 * b 1 * b 2 * \tau^{b 2-1} * e^{-b 1 * \tau^{b 2}}$ & $\mu(\tau)=b 0 *\left(1-e^{-b 1 * \tau^{b 2}}\right)$ \\
\hline Exponential Distribution & $\lambda(\tau)=b 0 * b 1 * e^{-b 1 * \tau}$ & $\mu(\tau)=b 0 *\left(1-e^{-b 1 * \tau}\right)$ \\
\hline Gamma Distribution & $\lambda(\tau)=b 0 * b 1^{2} * \tau * e^{-b 1 * \tau}$ & $\mu(\tau)=b 0 *\left(1-\left((1+b 1 * \tau) * e^{-b 1 * \tau}\right)\right)$ \\
\hline Power Distribution & $\lambda(\tau)=b 0 * b 1 * \tau^{b 1-1}$ & $\mu(\tau)=b 0 * \tau^{b 1}$ \\
\hline Geometric Distribution & $\lambda(\tau)=\frac{b 0 * b 1}{1+b 1 * \tau}$ & $\mu(\tau)=b 0 * \ln (1+b 1 * \tau)$ \\
\hline Inverse Linear Distribution & $\lambda(\tau)=b 0 * \frac{1}{2 * \sqrt{b 1+\tau}}$ & $\mu(\tau)=\mathrm{b} 0 *(\sqrt{\mathrm{b} 1+\tau}-\sqrt{\mathrm{b} 1})$ \\
\hline
\end{tabular}

For software reliability estimation, a U-plot is often used. The graph represents a line of unit slope. Any serious departure of the plot from this line is indicative of nonuniformity of the model to that data. The result obtained from all six software models are summarized in Table 7.3.

Table 7.3: Software Failure Results

\begin{tabular}{|l|l|l|l|}
\hline Model Name & $\begin{array}{l}\text { Failure Intensity } \\
\text { Failure/CPU Secs }\end{array}$ & $\begin{array}{l}\text { Reliability for next } \\
500 \text { CPU secs }\end{array}$ & $\begin{array}{l}\text { Sum of Squares of } \\
\text { Errors }\end{array}$ \\
\hline Weibull Distribution & 0.00002 & 0.9881 & 97565.6 \\
\hline Exponential Distribution & 0.00023 & 0.8934 & 8968.227 \\
\hline Gamma Distribution & 0.00007 & 0.9668 & 41992.43 \\
\hline Power Distribution & 0.00072 & 0.6984 & 7709.368 \\
\hline Geometric Distribution & 0.00051 & 0.7761 & 2467.949 \\
\hline Inverse Linear Distribution & 0.00076 & 0.6843 & 11457.86 \\
\hline
\end{tabular}


A sample STORE screen for the exponential model is shown in Figure 7.8. The figure shows a portion of the raw data along with reliability values, the growth curve, failure intensity plot, and the $U$ plot. The reliability value calculated for $X_{2}$ from the result screen is 0.8934

\begin{tabular}{|c|c|c|c|c|c|c|}
\hline & & & & & & \\
\hline \begin{tabular}{|l|} 
Failure Nos. \\
\end{tabular} & Failure Data & Failure Intensity & Reliability & CDF & KS Distance & Empiric \\
\hline 1 & 3 & 0.00494 & 0.08458 & 0.0001 & 0.00725 & 0.0 \\
\hline 2 & 33 & 0.004935 & 0.0848 & 0.00115 & 0.01356 & 0.0 \\
\hline 3 & 146 & 0.004916 & 0.08562 & 0.00507 & 0.01699 & 0.0 \\
\hline 4 & 227 & 0.004902 & 0.08622 & 0.00787 & 0.02154 & 0.0 \\
\hline 5 & 342 & 0.004882 & 0.08707 & 0.01183 & 0.02493 & 0.0 \\
\hline 6 & 351 & 0.004881 & 0.08713 & 0.01214 & 0.03197 & 0.0 \\
\hline 7 & 353 & 0.00488 & 0.08715 & 0.01221 & 0.03926 & 0.0 \\
\hline 8 & 444 & 0.004865 & 0.08782 & 0.01534 & 0.04349 & 0.0 \\
\hline 9 & 556 & 0.004846 & 0.08866 & 0.01917 & 0.04701 & 0.0 \\
\hline 10 & 571 & 0.004843 & 0.08877 & 0.01968 & 0.05385 & 0.0 \\
\hline 11 & 709 & 0.00482 & 0.08981 & 0.02438 & 0.05650 & 0.0 \\
\hline 12 & 759 & 0.004812 & 0.09018 & 0.02607 & 0.06216 & $0.0_{-1}^{-1}$ \\
\hline a & & & & & & ही \\
\hline
\end{tabular}

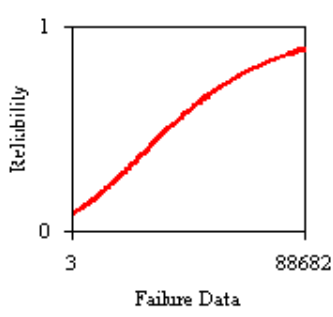

Reliability Growth Curve

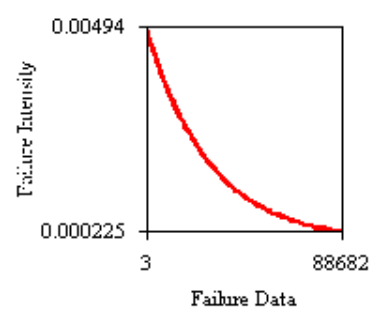

Failure Intensity Curve
Reliability of the software for next 500 unit time with failure intensity = 00023 is .8934

Sum of Square of Errors is 8968.227

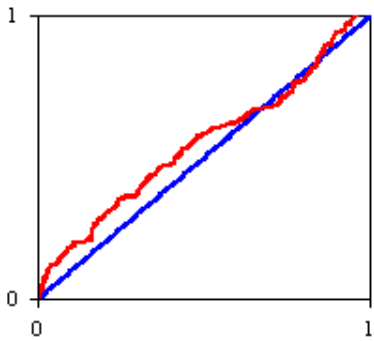

U -Plot

Figure 7.8: Sample STORE Screen for the Exponential Model (Software)

\subsubsection{Calculation of Hardware Reliability - Complete Data}

Figure 7.9 shows the screen of hardware reliability calculation form. In this form, the data can be read from a file, which can be opened using the directory and file menus on lower left side. Once the data is read, it automatically displays number of failures and cumulative failure time. After opening the file, the user is required to select one of the four distributions given and enter mission time. Once this is done, then by clicking the "Reliability" button, the program calculates the reliability and displays the value in the text box. Along with this MTTF, failure intensity and test statistics are also computed.

Consider the following failure data for hardware component $\mathrm{X}_{3}$, obtained from [4]. It represents time to failure (in hours) of a certain component. Let us consider this as 
complete data.

Table 7.4: Data for Hardware Reliability

\begin{tabular}{|l|l|l|l|l|l|l|l|l|l|}
\hline 50.1 & 20.9 & 31.1 & 96.5 & 36.3 & 99.1 & 42.6 & 84.9 & 6.2 & 32.0 \\
\hline 30.4 & 87.7 & 14.2 & 4.6 & 2.5 & 1.8 & 11.5 & 84.6 & 88.6 & 10.7 \\
\hline
\end{tabular}

The four hardware models were applied to the above data. The results obtained from each model are summarized in Table 7.5. Based on the test statistics the exponential model is most appropriate for the given data set. STORE determines the appropriate test statistics and the Chi square value.

Table 7.5: Results of Hardware Reliability Models

\begin{tabular}{|c|c|c|c|c|}
\hline Model & Parameters & $\begin{array}{c}\text { Reliability } \\
\text { for a } 15 \text { hour. } \\
\text { mission. }\end{array}$ & Test Statistic & Hypothesis \\
\hline Exponential & $\begin{array}{l}\text { b0 }(\lambda) \\
=0.023915\end{array}$ & 0.6985 & $\begin{array}{l}\text { Bartlett's Test: } \\
18.258\end{array}$ & Accepted \\
\hline Normal & $\begin{array}{l}\mathrm{b} 0(\mathrm{~m})=41.85 \\
\mathrm{~b} 1(\mathrm{~s})=35.22\end{array}$ & 0.7770 & $\begin{array}{l}\text { KS test value: } \\
0.1875\end{array}$ & $\begin{array}{l}\text { Not } \\
\text { accepted }\end{array}$ \\
\hline LogNormal & $\begin{array}{l}\mathrm{b} 0(\mathrm{~m})=1.217 \\
\mathrm{~b} 1(\mathrm{~s})=24.4571\end{array}$ & 0.6563 & $\begin{array}{l}\text { KS test } \\
\text { value: } 1.0002\end{array}$ & $\begin{array}{l}\text { Not } \\
\text { accepted }\end{array}$ \\
\hline Weibull & $\begin{array}{l}\mathrm{b} 0(\gamma)=0.924 \\
\mathrm{~b} 1(\beta)=44.1003\end{array}$ & 0.6911 & $\begin{array}{l}\text { Mann's Test: } \\
0.874\end{array}$ & $\begin{array}{l}\text { Not } \\
\text { accepted }\end{array}$ \\
\hline
\end{tabular}




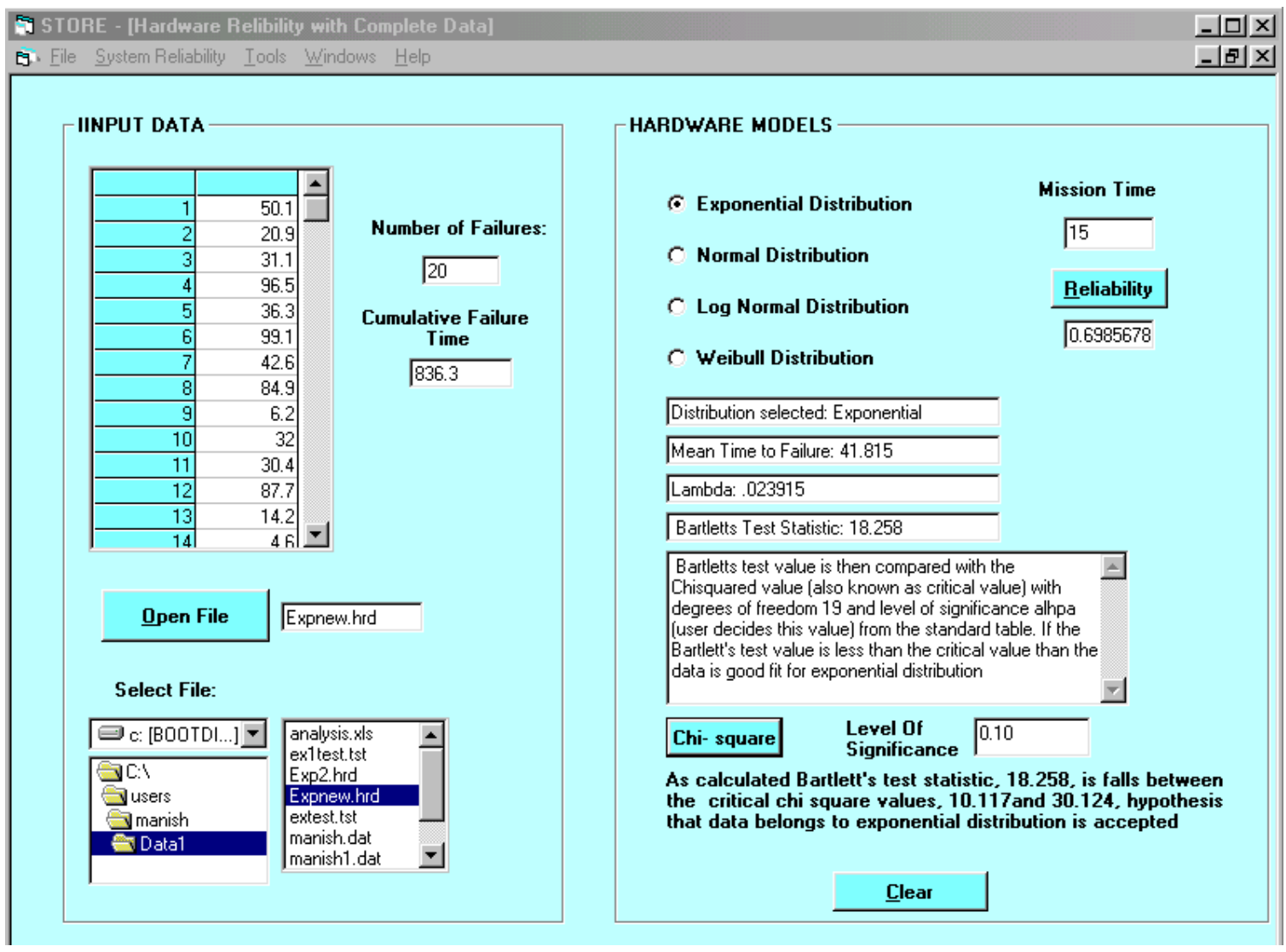

\section{Figure 7.9: Sample STORE Screen for the Exponential Model (Hardware)}

We find that only exponential model is accepted. Hence we consider the reliability of the component $\mathrm{X}_{3}$ as 0.6985 .

\subsubsection{Calculation of Hardware Reliability - Censored Data}

Let us assume that for component $\mathrm{X}_{4}$, we have censored data, with Type I censoring. That is there are total 40 observations, but we take observation till 32 components fail as shown in Table 7.5. The failure data was obtained from [1].

Table 7.5: Data for Hardware Reliability - Censored Data

\begin{tabular}{|l|l|l|l|l|l|l|l|l|l|l|l|l|l|l|l|}
\hline 117 & 279 & 128 & 148 & 156 & 17 & 236 & 94 & 182 & 172 & 112 & 117 & 119 & 194 & 297 & 49 \\
\hline 70 & 155 & 113 & 252 & 86 & 175 & 174 & 250 & 68 & 87 & 290 & 203 & 95 & 263 & 183 & 127 \\
\hline
\end{tabular}

The three hardware models were applied to the above data. The results obtained from each model are summarized in Table 7.6. Based on the test statistics the weibull model is most appropriate for the given data set. STORE determines the appropriate test statistics, the Chi square value and the F-values. 
Table 7.6: Results of Hardware Reliability Models

\begin{tabular}{|c|c|c|c|c|}
\hline Model & Parameters & $\begin{array}{l}\text { Reliability for a } 15 \\
\text { hour mission. }\end{array}$ & Test Statistic & Hypothesis \\
\hline Exponential & $\mathrm{b} 1=0.00418$ & 0.603 & Bartlett's Test: 7.516 & Not Accepted \\
\hline Normal & $\begin{array}{l}\mathrm{b} 0=-1.863 \\
\mathrm{~b} 1=0.009953\end{array}$ & 0.9631 & $\begin{array}{l}\text { KS test value: } \\
0.1195\end{array}$ & Not accepted \\
\hline Weibull & $\begin{array}{l}\mathrm{b} 0=-10.273 \\
\mathrm{~b} 1=1.977\end{array}$ & 0.654 & Mann's Test: 1.007 & Accepted \\
\hline
\end{tabular}

The sample screen using Weibull distribution is shown in the Figure 7.10. It shows that Weibull distribution is accepted and the reliability of the component $\mathrm{X}_{4}$ is 0.654 .

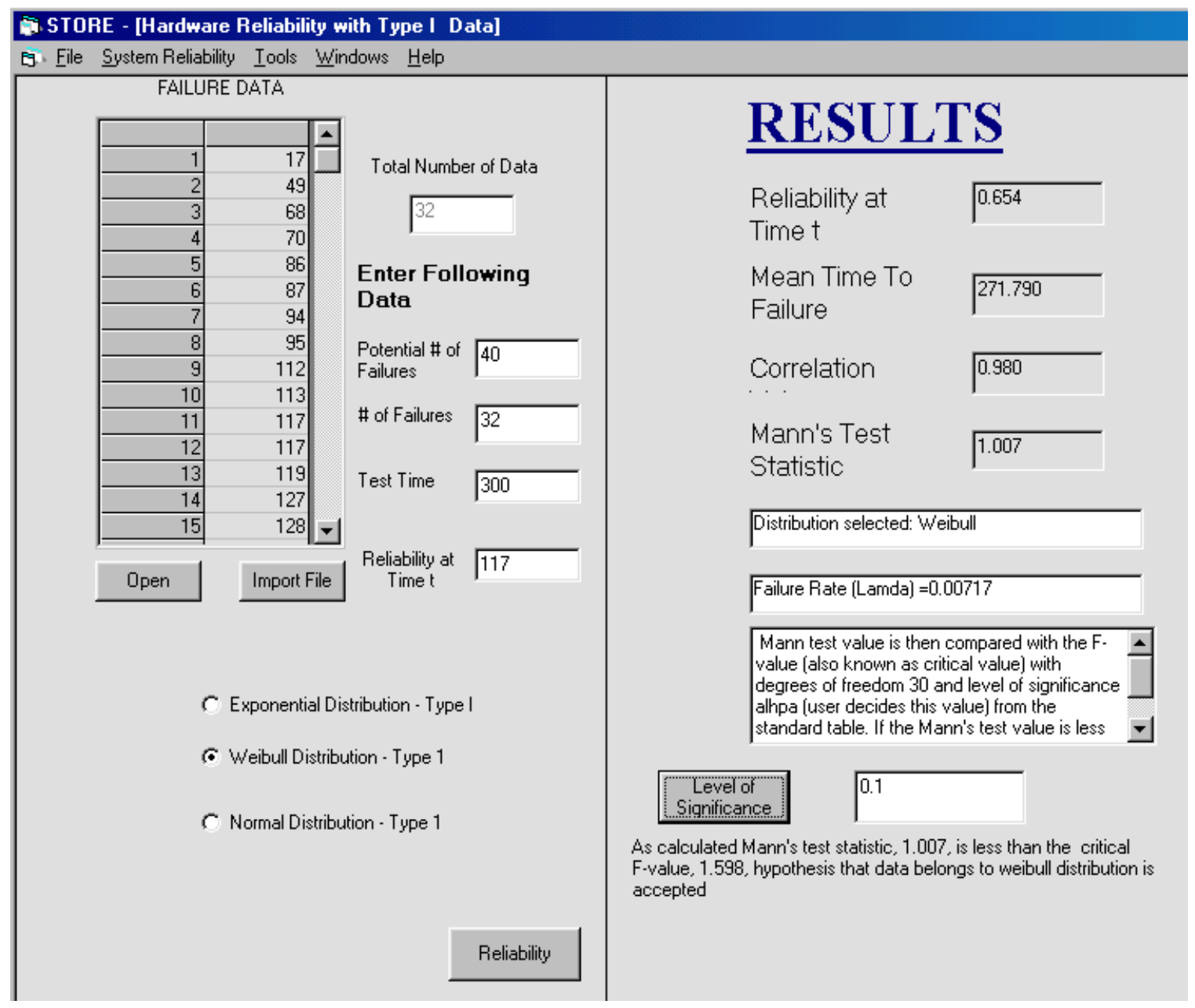

\subsubsection{Calculation of System Reliability}


Once the user knows the reliability values for all the components. STORE can calculate the system reliability using the tie set algorithm. Now we know that reliabilities for the components are as follows: $X_{1}=0.93, X_{2}=0.8934, X_{3}=0.6985, X_{4}=$ 0.654 and $\mathrm{X}_{5}=0.98$.

The tie sets and the system reliability is displayed in the system. The above reliability block diagram was taken from [21]. The tie sets obtained by STORE were the same as in [21]. Since we assumed different components as hardware and software and took failure data from other references, system reliability is not the same as in [21]. The tie sets obtained are: $\mathrm{T}_{1}=\mathrm{X}_{5}, \mathrm{X}_{2} ; \mathrm{T}_{2}=\mathrm{X}_{5}, \mathrm{X}_{3}, \mathrm{X}_{1} ; \mathrm{T}_{3}=\mathrm{X}_{5}, \mathrm{X}_{4}, \mathrm{X}_{1}$ and the system reliability as 0.97805 . The system reliability calculation screen is shown in Figure 7.11.

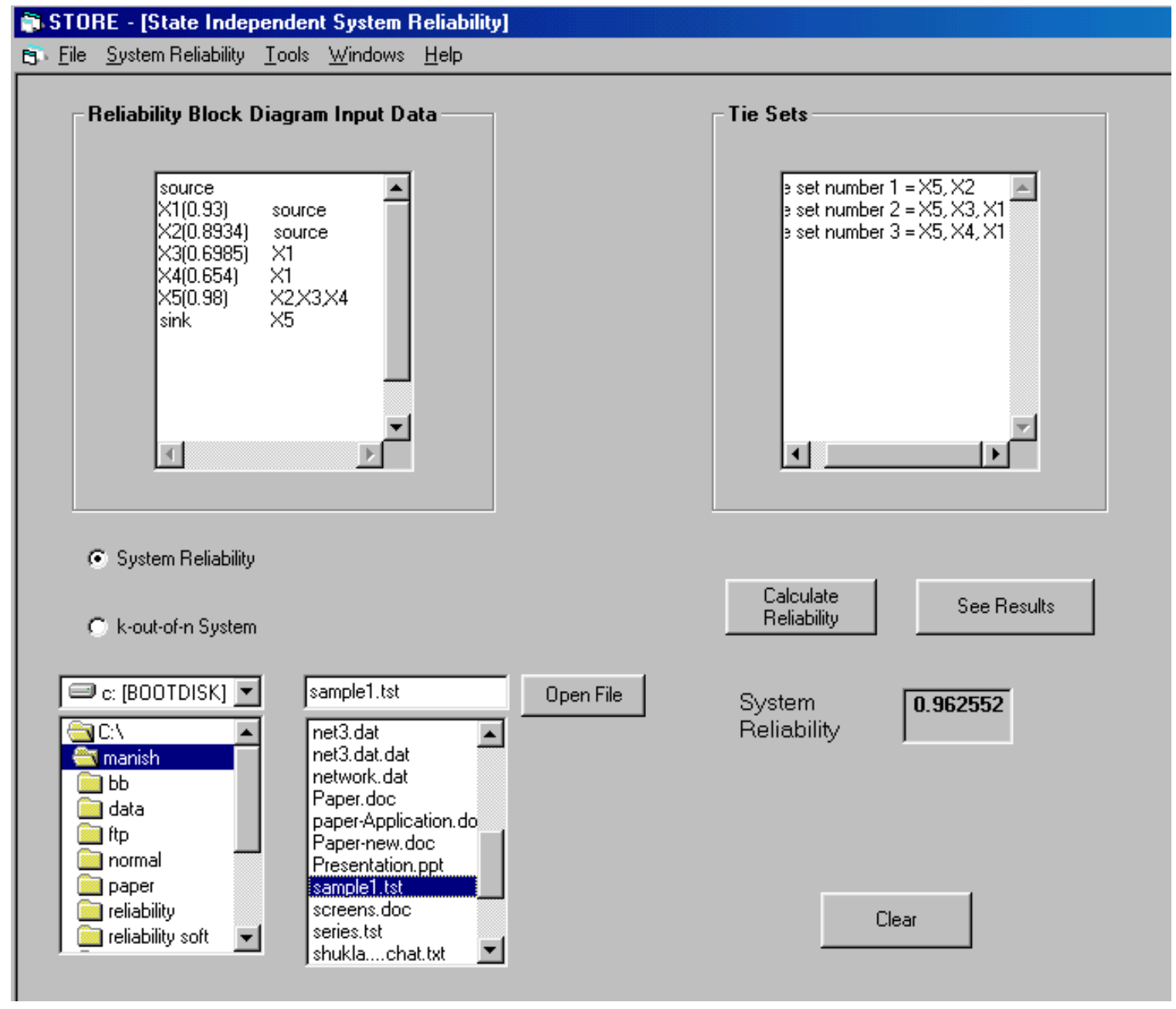

Figure 7.11: System Reliability Screen

\subsection{Example 5: State Dependent Systems}


Consider the following problem from example 6.2 of [4] for Standby system: An active generator has a failure rate (failures per day) of 0.01. An older standby generator has a failure rate of 0.001 while in standby and a failure rate of 0.10 when online. Determine the system reliability for a planned 30-day use and compute the system MTTF.

This problem can be solved using STORE. The solution is shown in Figure 7.12.

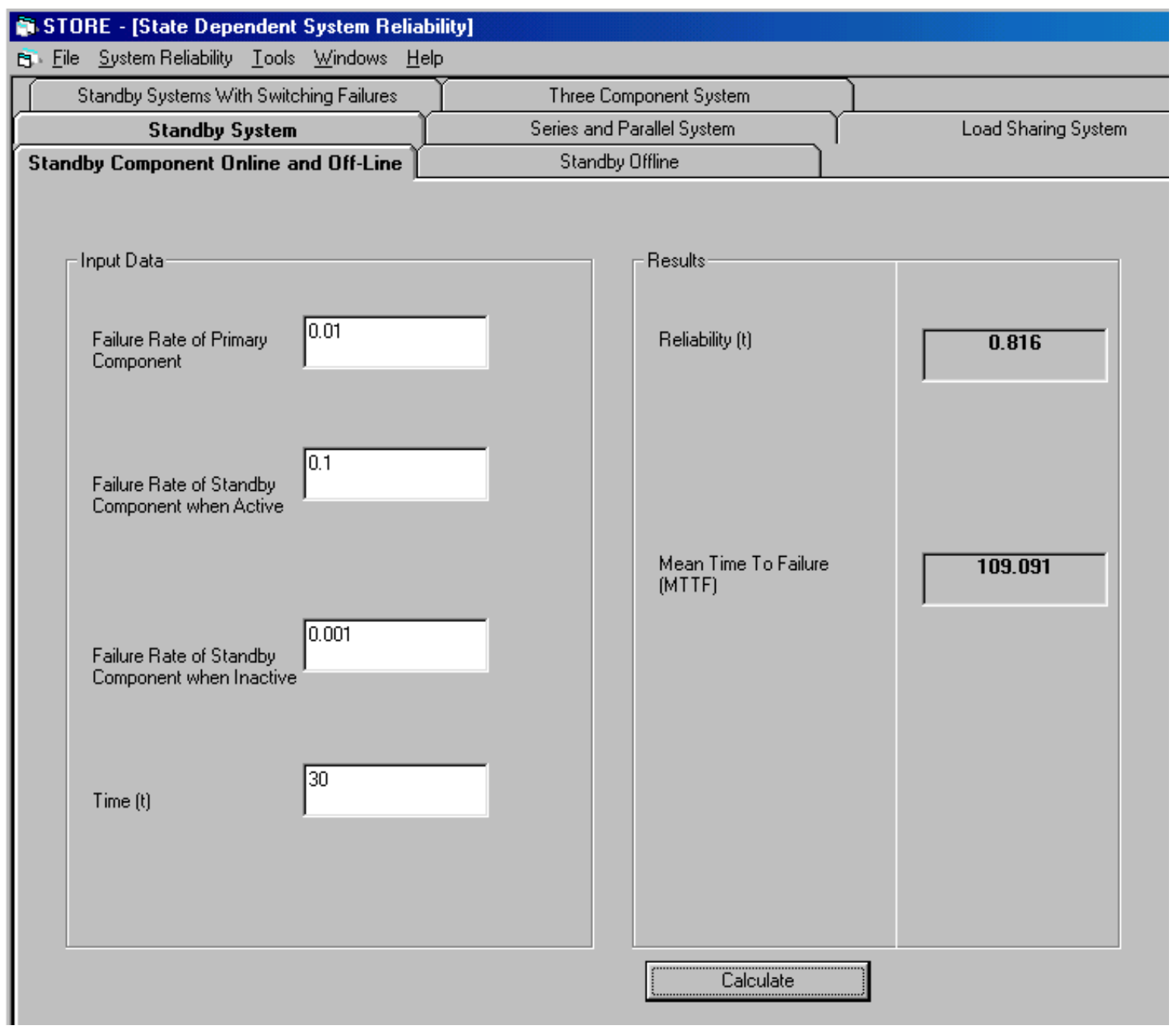

Figure 7.12: Sample STORE Screen Showing State Dependent System Reliability for Standby system.

The user is required to input failure rate of active component, standby component online and offline, with time in state 1 . The tool then calculates the system reliability and MTTF as 0.816 and 109.091 respectively, which is same as in [8].

STORE uses the computational formulae given in [8] for state dependent systems for system reliability and MTTF calculation. STORE is capable of calculating system reliability and MTTF for Markov Analysis, Load Sharing System, Standby Systems, 
Standby Systems with Switching Failures and Three Component Systems. The results using STORE for all these types is shown in Table 7.7, which is same as examples $6.1,6.2,6.5$ and 6.6 of [8].

Table 7.7: Reliability and MTTF for State Dependent Systems

\begin{tabular}{|l|c|c|}
\hline Type & Reliability & MTTF \\
\hline Standby Systems & 0.816 & 109.091 \\
\hline $\begin{array}{l}\text { Standby Systems with } \\
\text { Switching Failures }\end{array}$ & 0.808 & 108.18 \\
\hline Load Sharing & 0.931 & 60 \\
\hline Three Component & 0.744 & 857.143 \\
\hline
\end{tabular}




\section{Chapter 8: Conclusions and Future Work}

\subsection{Conclusion}

The aim of this research was to develop a software tool to estimate hardware, software and system reliability. In hardware reliability, problems with both complete and censored data were considered. And in system reliability, two component state dependent system reliability was implemented. The author did not come across any literature where such an integrated tool was presented or described. The software was implemented in Visual Basic. It provides a user-friendly environment, where small sets of easily comprehend input factors are required. Using this software, data entry, data update and retrieval can be performed in a short period of time.

The following models were implemented under hardware reliability for censored data were:

i. Exponential

ii. Normal

iii. Weibull

The models for estimating hardware reliability for complete data were:

i. Exponential

ii. Normal

iii. Weibull

iv. Log Normal

The models for estimating software reliability were:

i. Exponential

ii. Weibull

iii. Gamma

iv. Power

v. Logarithmic

vi. Inverse Linear

Apart from the above models several goodness of fit tests, maximal likelihood tests and other statistical tests were incorporated to aid user in deciding which is the best model for that particular data set. In addition to software and hardware reliability calculations, system reliability estimation was incorporated for both state dependent and 
independent systems.

In state dependent systems, following types of systems were implemented:

i. Markov Analysis

ii. Load Sharing System

iii. Standby Systems

iv. Standby Systems with switching failures

v. Three-component systems

For state independent system reliability estimation, tie set algorithm was used as described in [21] for given block diagram.

\subsection{Future Work}

The software developed was able to perform calculations for hardware reliability, software reliability, state dependent system reliability and state independent system reliability. However as the respective areas of application being very vast, the software can be enhanced in the following areas:

1. Developing a Graphical User Interface which will allow the user to

- Build a reliability block diagram to show the network of components.

- Dynamically add, move or delete components in a system.

- Analyze the calculated system reliability by rearranging the network to enhance the reliability and decide on adding parallel or standby components.

- Design a system with predefined reliability.

2. Develop a more efficient algorithm for tie set calculation.

3. Incorporate State Dependent Systems for $\mathrm{n}$ components from the existing twocomponent system using Markov analysis.

4. Implement other models for Software Reliability.

5. Implement Software Reliability models for Censored data

6. Provide a comprehensive Help function.. 


\section{References}

1 Ahluwalia R S, "Quality and Reliability Engineering", Lecture Notes, West Virginia University, 1998.

2 Ahluwalia R S, Parekh M, "Software Reliability Estimation using Exponential \& Gamma Models", Manufacturing Tech. Beyond 2000, Bangalore, India, 1999.

3 Boukas, Yang, "Cost effective maintenance management, Productivity improvement and Downtime reduction", 1983 Noyes Publication.

4 Dimitri Kececioglu, "Reliability and Life Testing Handbook, Vol.1”, 1993, PTR Prentice-Hall, Inc.

5 Dungarpur J S and Jack N, “ Optimizing System Availability under Minimal Repair with Non-negligible Repair and Replacement", 1993, Journal of Operational Research Society, Vol.44, No. 11, pp. 1097-1103.

6 Duane J T., "Learning Curve Approach to Reliability Monitoring", IEEE Transactions on Aerospace Vol. 2 pp. 563-566, 1964.

7 DeMercado J, Spyratos N, and Bowen B A, "A Method for Calculation of Network Reliability,” IEEE Trans. Reliability, Vol. R-25 pp. 71-77, June 1976.

8 Ebeling C E, “ An Introduction to Reliability and Maintainability Engineering”, McGraw Hill, 1997.

9 Elsayed A. E, "Reliability Engineering”, Addison Wesley Longman, June 1996.

10 Flehinger B J, "System Reliability as a Function of System Age: Effects of Intermittent Component Usage and Periodic Maintenance", 1960, Operations Research, 1960, Vol. 8, No. 1, pp. 30-44.

11 Gotkas, Yavuz, "Integrated Reliability, Availability, Maintainability cost modelling for the manufacturing design system", PhD. Dissertation, West Virginia University, 1995.

12 Grady, R.B., and Casewell, D.L., "Software Metrics: Establishing A Company-Wide Program”, Prentice-Hall, Englewood Cliffs, New Jersy, 1987.

13 Hudson G R., "Program Errors as Birth and Death Process", System Development Corporation, Report SP -3011, Santa-Monica, CA, 1967.

14 Igor A. Ushakov, and Robert A. Harrison, "Handbook of Reliability Engineering", 1994, John Wiley and Sons.

15 Jelinski Z, and Moranda P B., "Software Reliability Research", Statistical Computer 
Performance Evaluation, Academic, New York, pp. 465-484, 1972.

16 Jardine A K S, "Maintenance, Replacement and Reliability", 1973, John Wiley and Sons.

17 Littlewood B and Verrall J L., "A Bayesian Reliability Growth Model for Computer Software”, Journal Royal Statistical Society -Series C, 22(3), pp.332-346, 1973.

18 Moranda P B, "Predictions of Software Reliability During Debugging”, Proceedings Annual Reliability and Maintainability Symposium Washington DC, pp. 327-332, 1975.

19 Musa J D, "Validity of Execution-Time Theory of Software Reliability", IEEE Transactions on Reliability, Vol. R-28, pp. 181-191, August 1979.

20 Musa J D, A Iannino, K Okumoto, "Software Reliability: Measurement, Prediction, Application”, McGraw- Hill, New- York, 1987.

21 Nelson A C, Batts J R, and Beadles R L, "A Computer Program Approximating System Reliability,” IEEE Trans. Reliability, Vol. R-19 pp. 6165, May 1970.

22 Nikora A P, "An Experiment in Determining Software Reliability Applicability", Toulouse, France, International Symposium on Software Reliability Engineering, October 1995.

23 Nikora A P, "A JPL Software Reliability study and a Windows-Based Software Reliability Tool", Claremount, California, USA, November 1993.

24 Parekh, M, "Development of Decision Support System for Reliability", MS Thesis, West Virginia University, 1999.

25 Patrick D T O'Connor; "Practical Reliability Engineering”, John Wiley \& Sons, 1991.

26 Raze J, Nelson J J, Simrad D J, Bradley M, "Reliability Models for Mechanical Equipment", 1987 Proceedings Annual Reliability and Maintainability Symposium, pp. 130-133.

27 Schoutten F A and Vanneste S G, " Simple Control Policies for a Multicomponent Maintenance Systems”, 1993, Operations Research Journal, Vol.41, No.6, pp. 11251136.

28 Shooman, M L; “Probabilistic Models for Software Reliability Prediction”, Statistical Computer Performance Evaluation, Academic, New York, pp. 485-502, 1972.

29 Schick G J, and Wolverton R W., "Assessment of Software Reliability”, Proceedings Operations Research, Physica-Verlag, Wurzburg-Wein, pp. 395-422.

30 Schick G J, and Wolverton R W., “An Analysis of Competing Software Reliability Models", IEEE Transactions on Software Engineering, SE-(4), pp. 104-120, 1978. 
31 Schneidewind N F., "An Approach to Software Reliability Prediction and Quality Control", Fall joint Computer Conference, AFPIS conference Proceedings, 41 AFPIS press, Montvale, NJ, pp.837-847, 1972.

32 Schneidewind N F., "Analysis of Error Processes in Computer Software", Proceedings 1975 International Conference on Reliable Software Los Angeles, pp. 337-346, 1975.

33 Schneider, D I, “An Introduction to Programming Using Visual Basic 6.0”, Prentice Hall, Fourth Edition, 1999.

34 Wagoner, W L; "The Final Report on Software Reliability Measurement Study", Aerospace Corporation, Report TOR -0074(4112)-1.

35 Yuan, Kai, Cai, "Towards a conceptual framework of software run reliability modeling", Information Sciences, July 2000, Vol. 126 Issue 1-4, p137, 27p. 


\section{Appendix I: Bartlett's Test for Exponential Distribution}

A specific test for fitting exponential distribution is Bartlett's test [1]. The hypotheses are:

Ho: Failure times are exponential

Ha: Failure times are not exponential

The test statistic is

$B=\frac{2 . n \cdot\left(\ln \left((1 / n) \cdot\left(\sum_{i=1}^{n} t_{i}\right)\right)-(1 / n) \cdot \sum_{i=1}^{n} \ln \left(t_{i}\right)\right)}{1+(n+1) /(6 . n)}$

Where $t_{i}=$ time of failure of ith unit.

$\mathrm{n}=$ number of failures

the test statistic B under the null hypothesis has a chi-square distribution with $\mathrm{n}-1$ degrees of freedom. In this test if,

$\chi^{2}(1-\alpha / 2, n-1)<B<\chi^{2}(\alpha / 2, n-1)$

then null hypothesis is accepted; otherwise the alternative hypothesis is accepted. 


\section{Appendix II: Kolmogorov-Smirnov Test}

A goodness of fit test for Normal and Log normal distribution is KolmogorovSmirnov test [1]. The hypotheses for the test are:

Ho: The failure time are normal (lognormal)

Ha: The failure times are not normal (lognormal)

The test statistic is

$\mathrm{D}_{\mathrm{n}}=\max \left\{\mathrm{D}_{1}, \mathrm{D}_{2}\right\}$

where $D_{1}=\left(\phi\left(\frac{t_{i}-\bar{t}}{s}\right)-\frac{i-1}{n}\right)$ and $D_{2}=\left(\frac{1}{n}-\phi\left(\frac{t_{i}-\bar{t}}{s}\right)\right)$

Where

$\bar{t}=\sum_{i=1}^{n} \frac{t_{i}}{n} \quad$ and $\quad s^{2}=\frac{\sum_{i=1}^{n}\left(t_{i}-\bar{t}\right)^{2}}{n-1}$

If $D_{n}<D_{\text {crit }}$, then accept Ho otherwise accept Ha. The values of $D_{\text {crit }}$ may be found in the standard Kolmogorov-Smirnov table. 


\section{Appendix III: Mann's Test for Weibull Distribution}

A specific test for weibull failure distribution is a test developed by Mann, Schafer, and Singapurwalla [1]. The hypotheses are:

Ho: The failure times are of Weibull

Ha: The failure times are not of Weibull.

The test statistic is:

$$
M=\frac{k_{1} \sum_{i=k 1+1}^{n-1}\left(\ln \left(t_{i+1}\right)-\ln \left(t_{i}\right)\right) / M_{i}}{k_{2} \sum_{i=1}^{k 1}\left(\ln \left(t_{i+1}\right)-\ln \left(t_{i}\right)\right) / M_{i}}
$$

Where $\mathrm{k}_{1}=[\mathrm{n} / 2], \mathrm{k}_{2}=[(\mathrm{n}-1) / 2]$, $\mathrm{M}_{\mathrm{i}}=\mathrm{Z}_{\mathrm{i}}+1-\mathrm{Z}_{\mathrm{i}}$

$Z_{i}=\ln \left[-\ln \left(1-\frac{1-0.5}{n+0.25}\right)\right]$

And $[\mathrm{x}]$ is less than number $x . \mathrm{M}_{\mathrm{i}}$ is an approximation. If $\mathrm{M}>\mathrm{F}_{\text {crit }}$, then $\mathrm{Ha}$ is accepted. Values for $\mathrm{F}_{\text {crit }}$ may be obtained from tables of F-distribution if one lets the number of degrees of freedom for numerator to be $2 \mathrm{k}_{2}$ and the number of degrees of freedom for the denominator be $2 \mathrm{k}_{1}$. 


\section{Appendix IV: Reliability Block Diagram Input}

In STORE, a RBD of the system is inputted by stating component, its reliability and predecessor. Where source is the predecessor of $\mathrm{X} 1$ and $\mathrm{X} 1$ is of $\mathrm{X} 3$. The value in parentheses is the reliability value of the component. The format is as shown in figure below.

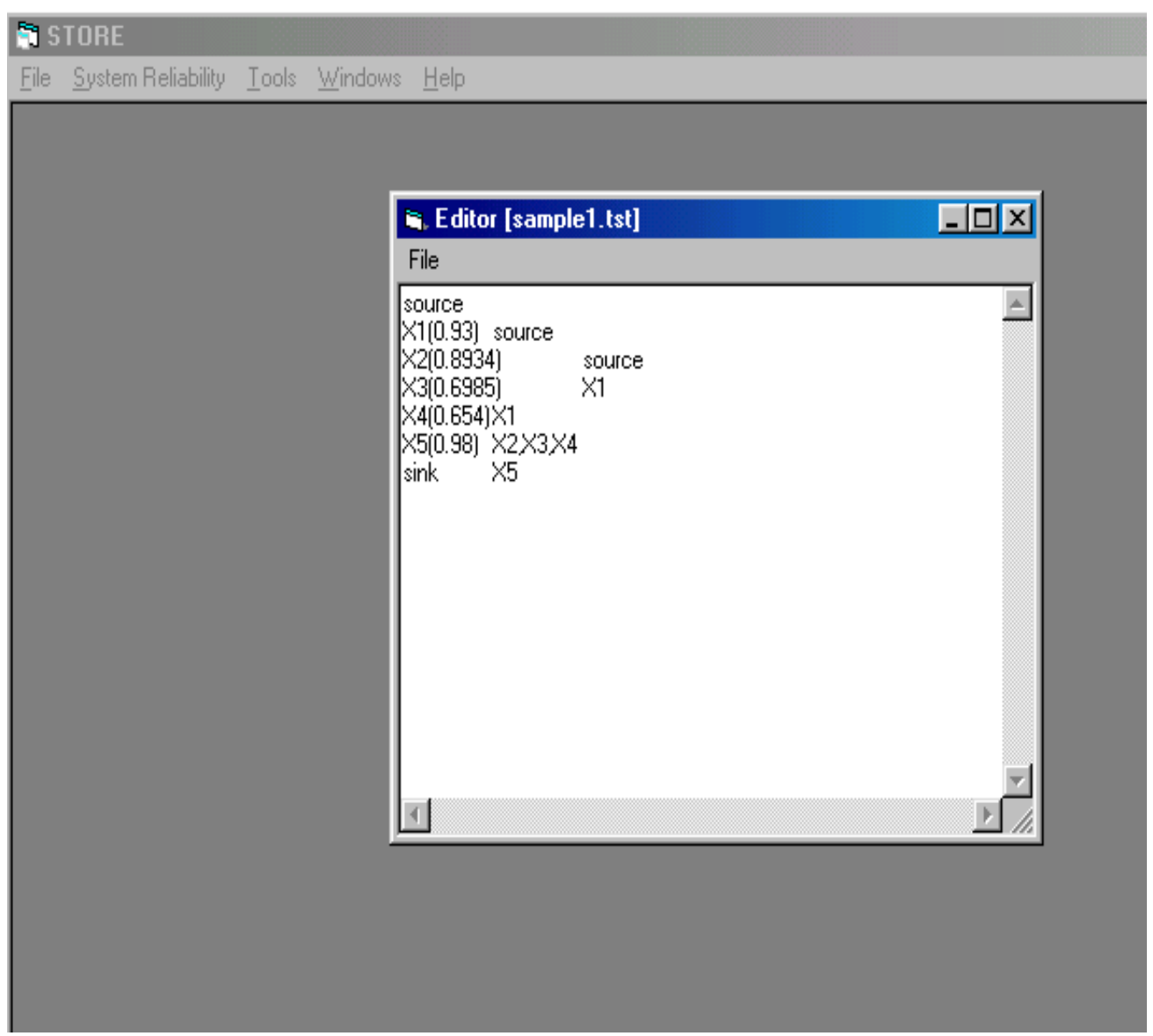




\section{Appendix V: Source Code}

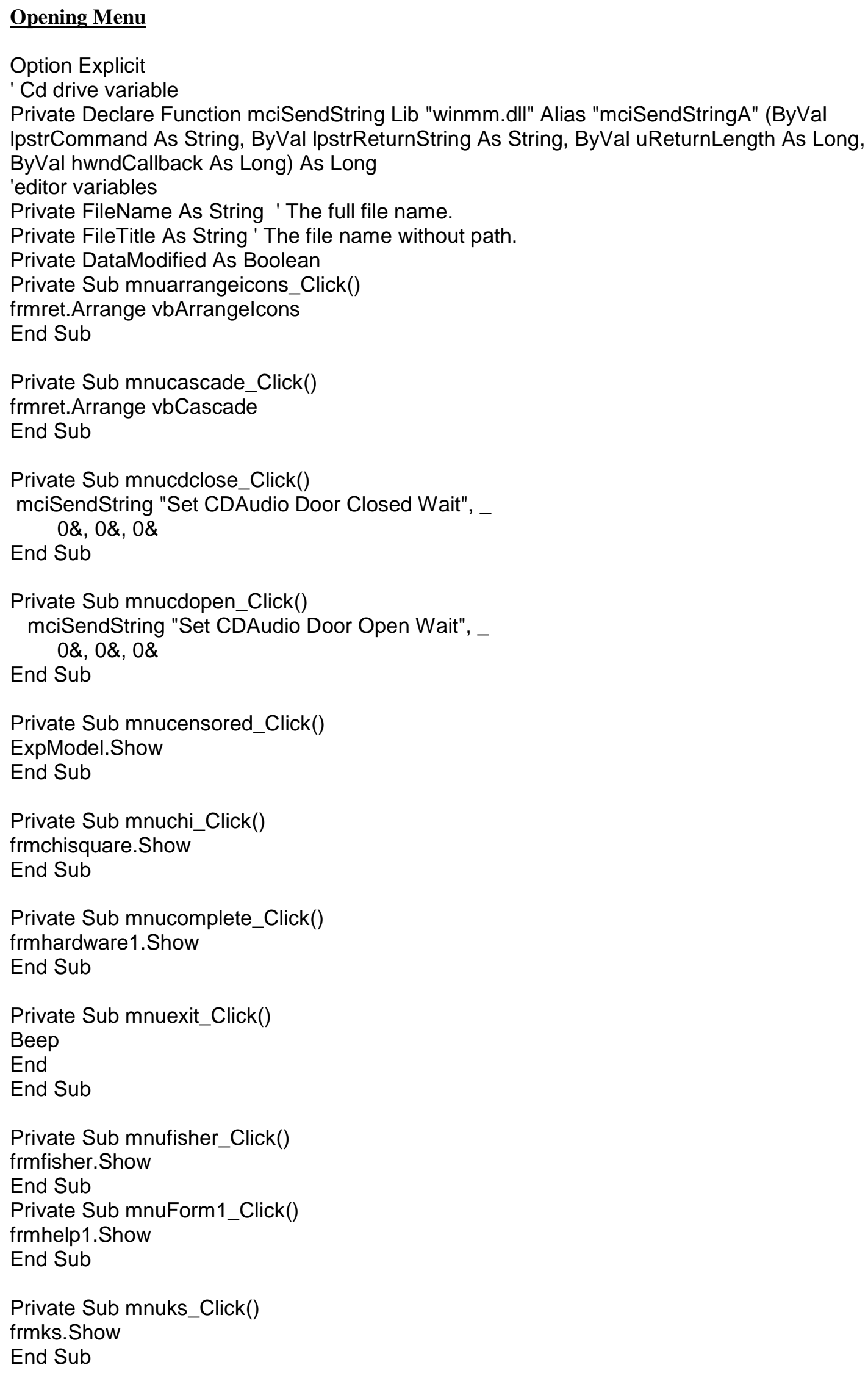




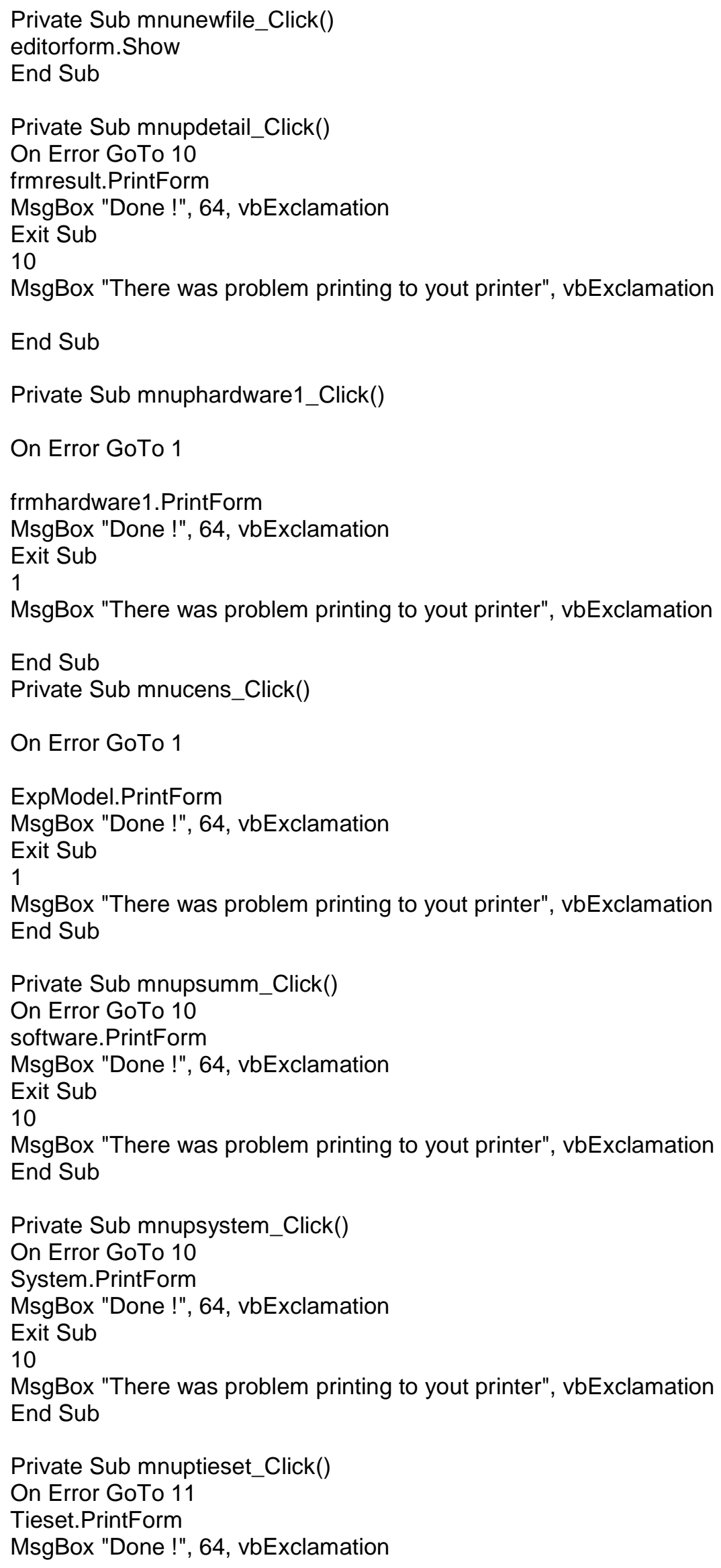




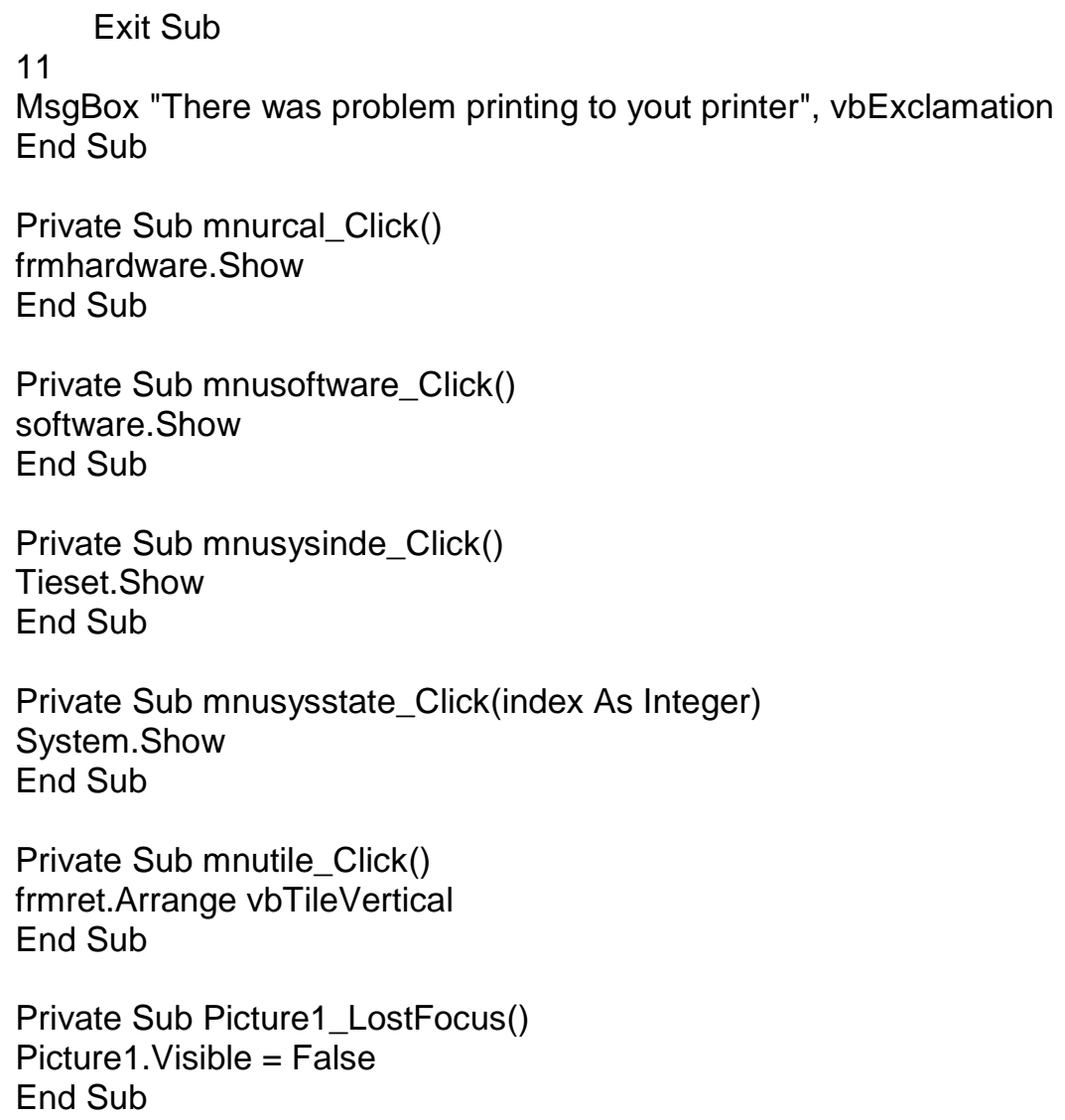

Data Editor

Option Explicit

Private FileName As String ' The full file name.

Private FileTitle As String ' The file name without path.

Private DataModified As Boolean

' Return True if the data is safe.

Private Function DataSafe() As Boolean

' No problem if the data is unmodified.

If Not DataModified Then

DataSafe $=$ True

Exit Function

End If

'See if the user wants to save changes.

Select Case MsgBox("The data has been modified. Do you want to save the changes?", vbYesNoCancel)

Case vbYes

' Save the data. Procedure SaveData

' will reset DataModified.

mnuesaveas Click

DataSafe $=$ Not DataModified

Case vbNo

' Discard the changes to the data.

DataSafe $=$ True

Case vbNo 


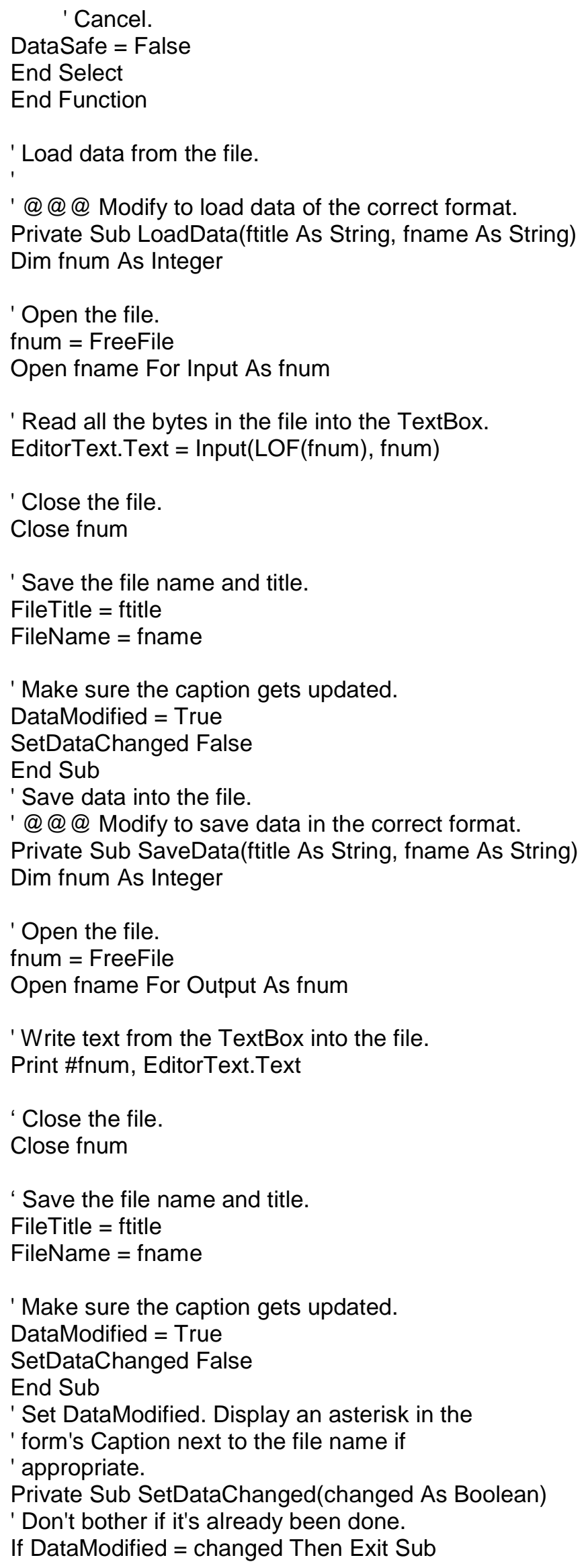




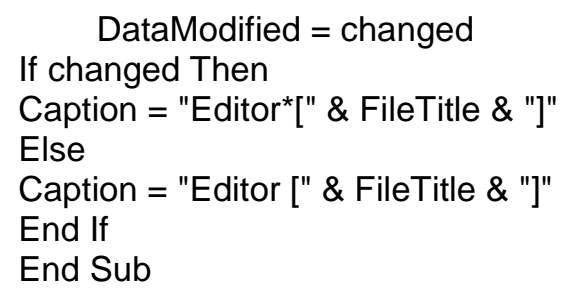

' Set the file dialog's path for the next time.

Private Sub SetDialogPath()

Dim file_path As String

' Remove characters from the right until the

' path ends in $\backslash$.

file_path = filedialog. FileName

Do While Right\$(file_path, 1) <> "।"

file_path = Left\$(file_path, Len(file_path) - 1)

Loop

filedialog.InitDir = file_path

' Save the directory in the registry.

'@@@ Change the application and section names.

SaveSetting "SimpleEditor", "Directories",

"SaveDir", filedialog.InitDir

End Sub

' Mark the data as modified.

'@@@ Call DataChanged whenever the user

'@@@ changes the data.

Private Sub EditorText_Change()

SetDataChanged True

End Sub

Private Sub Form_Load()

Dim wid As Single

Dim hgt As Single

' Get the last directory the program accessed.

' If there is no entry, use the App.Path.

'@@@ Change the application and section names.

filedialog.InitDir = GetSetting (

"SimpleEditor", "Directories",

"SaveDir", App.path)

End Sub

' Make sure the data is safe to unload.

Private Sub Form_QueryUnload(Cancel As Integer, UnloadMode As Integer)

Cancel $=$ Not DataSafe

End Sub

Private Sub Form_Resize()

EditorText.Move 0, 0, ScaleWidth, ScaleHeight

End Sub

' Unload the form.

Private Sub mnueexit_Click()

' Note: The QueryUnload event handler checks

' that the data is safe.

Unload Me 
' Start a new file.

Private Sub mnuenew_Click()

' Make sure the existing data is safe.

If Not DataSafe Then Exit Sub

'@@@Do whatever is necessary to start a

'@@@ new document.

EditorText.Text = "'"

' Save the file name and title.

FileTitle $=$ "'"

FileName $=" "$

' Make sure the caption gets updated.

DataModified $=$ True

SetDataChanged False

End Sub

' Open a file.

Private Sub mnueopen_Click()

' Make sure the existing data is safe.

If Not DataSafe Then Exit Sub

' Start in the application directory.

If filedialog.InitDir = "'" Then

filedialog. InitDir = App. path

'@@@ Set desired flags.

filedialog.Flags = cdlOFNFileMustExist + _ cdlOFNHideReadOnly + _

cdlOFNLongNames

'@@@ Set desired filters.

filedialog.Filter = "hardware $\left.\left({ }^{*}\right.$.hrd $)\right|^{*}$.hrd |"

filedialog. Filter $=$ filedialog. Filter + "software $\left.\left({ }^{*}\right.$.sft $)\right|^{*}$. sft |"

filedialog.Filterlndex $=0$

' Let the user select the file to open.

On Error Resume Next

filedialog.ShowOpen

If Err.Number $=$ cdlCancel Then

' The user canceled.

Exit Sub

Elself Err.Number $<>0$ Then

MsgBox "Error" \& Str\$(Err.Number) \& " selecting file." \&

vbCrLf \& Err.Description

Exit Sub

End If

On Error GoTo 0

' Set the dialog path for next time.

SetDialogPath

' Load the data.

LoadData filedialog.FileTitle, filedialog.FileName

End Sub 
' Save using the current file name.

Private Sub mnuesave_Click()

' If there's no file name, treat as Save As.

If FileTitle = "' Then

mnuesaveas_Click

Exit Sub

End If

' Save the data using the current file name.

SaveData FileTitle, FileName

End Sub

' Save using a new file name.

Private Sub mnuesaveas_Click()

' Start in the application directory.

If filedialog.InitDir = "' Then

filedialog.InitDir = App.path

'@@@ Set desired flags.

filedialog.Flags = cdlOFNPathMustExist +

cdlOFNHideReadOnly + _

cdlOFNLongNames

'@@@ Set desired filters.

filedialog.Filter $=$ "hardware $\left.\left({ }^{*}\right.$.hrd $)\right|^{*}$. hrd | "

filedialog.Filter $=$ filedialog. Filter + "software $\left.\left({ }^{*} . \mathrm{sft}\right)\right|^{*} .\left.\mathrm{sft}\right|^{\prime}$

filedialog.Filter $=$ filedialog.Filter + "Tieset Matrix $\left.\left({ }^{*}\right.$. tst $)\right|^{*}$.tst|"

filedialog. Filter = filedialog.Filter + "Cutset Matrix ( ${ }^{*}$.cst $)\left.\right|^{*}$.cst|"

filedialog.Filter $=$ filedialog. Filter + "Reliability Matrix $\left.\left({ }^{*}\right.$.rel $)\right|^{*}$.rel|"

filedialog. FilterIndex $=1$

' Let the user select the file to open.

On Error Resume Next

filedialog.ShowSave

If Err.Number $=$ collCancel Then

' The user canceled.

Exit Sub

Elself Err.Number $<>0$ Then

MsgBox "Error" \& Str\$(Err.Number) \& " selecting file." \&

vbCrLf \& Err.Description

Exit Sub

End If

On Error GoTo 0

' Set the dialog path for next time.

SetDialogPath

' Load the data.

SaveData filedialog.FileTitle, filedialog.FileName

End Sub

\section{State Independent System Reliability}

Dim hConsole As Long

Private Sub cmdopen_Click()

End Sub 


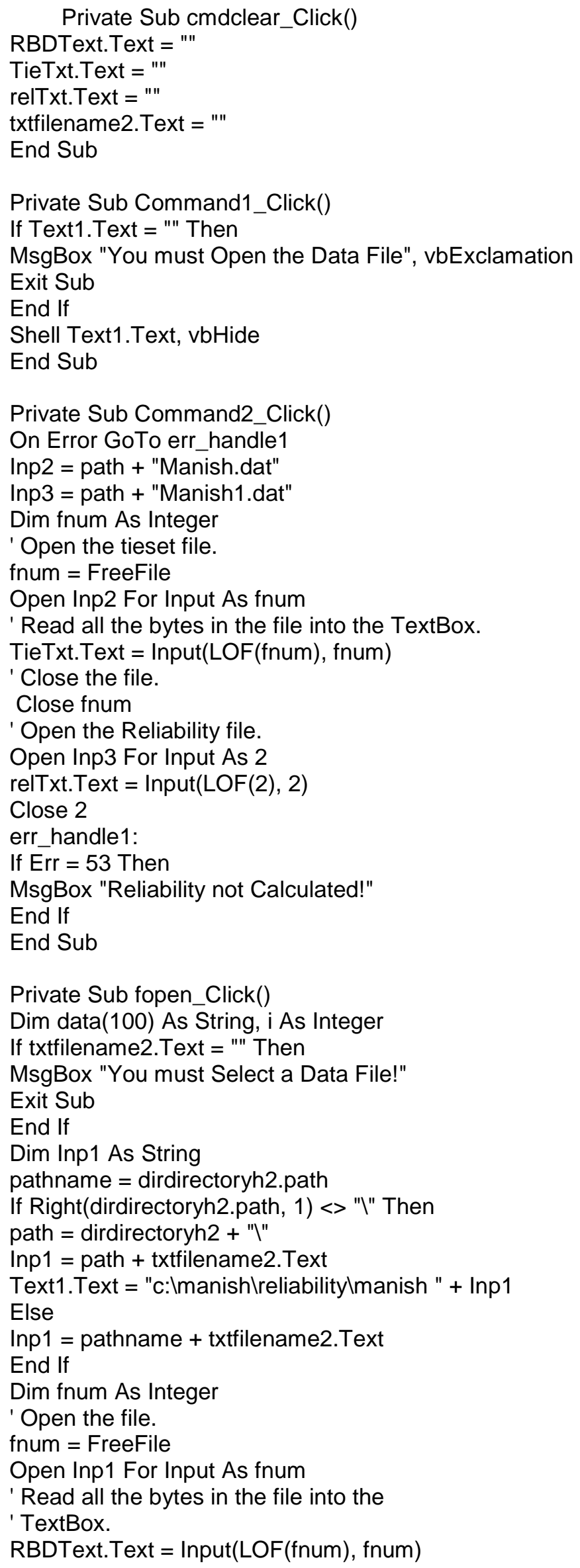




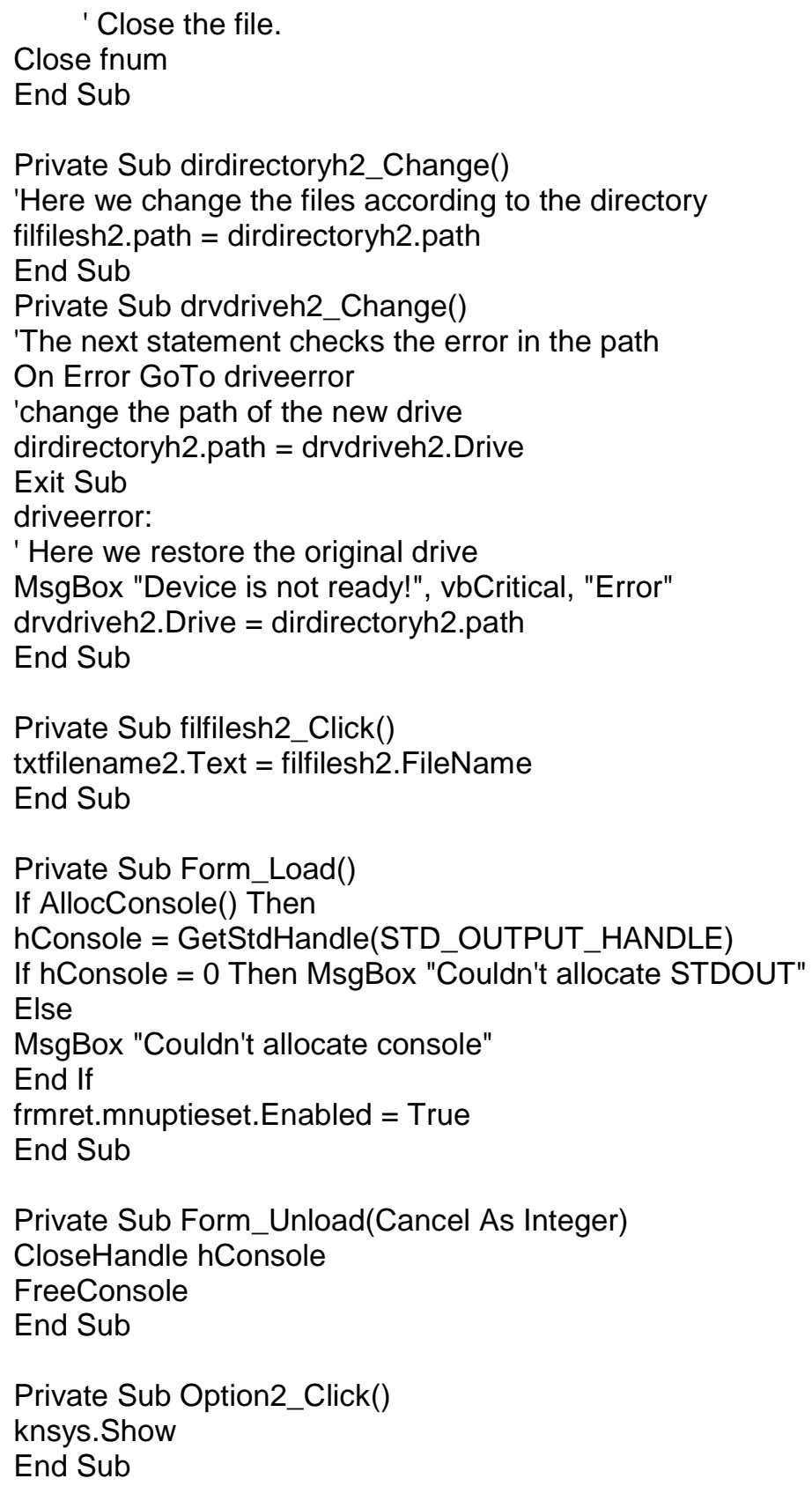

\section{State Dependent System Reliability}

Private Sub Cal_Click()

'Stand by sytem with failure rate for online and offline rel_SB $=\left(\operatorname{Exp}\left(-\operatorname{Val}\left(\mathrm{txtFR} \_\mathrm{SB}(2) . T e x t\right){ }^{*} \mathrm{Val}(\right.\right.$ Time_t $\left.\left.(0) \cdot T e x t)\right)\right)+(\operatorname{Val}(\mathrm{txtFR} S \mathrm{SB}(2) \cdot T$ ext $) /$

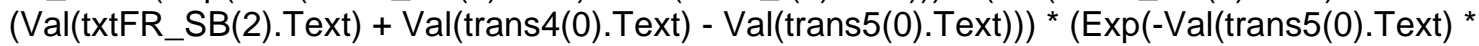
$\operatorname{Val}($ Time_t $(0) . T e x t))-\operatorname{Exp}\left(-(\operatorname{Val}(\operatorname{txtFR} S B(2) . T e x t)+\operatorname{Val}(\operatorname{trans} 4(0) . T e x t)){ }^{*} \mathrm{Val}(\operatorname{Time} \mathrm{t}(0)\right.$. Text $\left.\left.)\right)\right)$ rel3.Caption = Format(Str(rel_SB), "0.000")

mttf_SB $=\left(1 / \mathrm{Val}\left(\mathrm{txtFR} \_\mathrm{SB}(2)\right.\right.$. Text $\left.)\right)+\left(\mathrm{Val}\left(\mathrm{txtFR} \_\mathrm{SB}(2)\right.\right.$. Text $) /(\operatorname{Val}(\operatorname{trans5}(0) . T e x t)$ * $($ Val(txtFR_SB(2).Text) + Val(trans4(0).Text))))

mttf2.Caption = Format(Str(mttf_SB), "0.000")

End Sub

Private Sub Cal1_Click()

' Standby Systems with reduced failure rate

Dim mttf_SB As Double, rel_SB As Double 


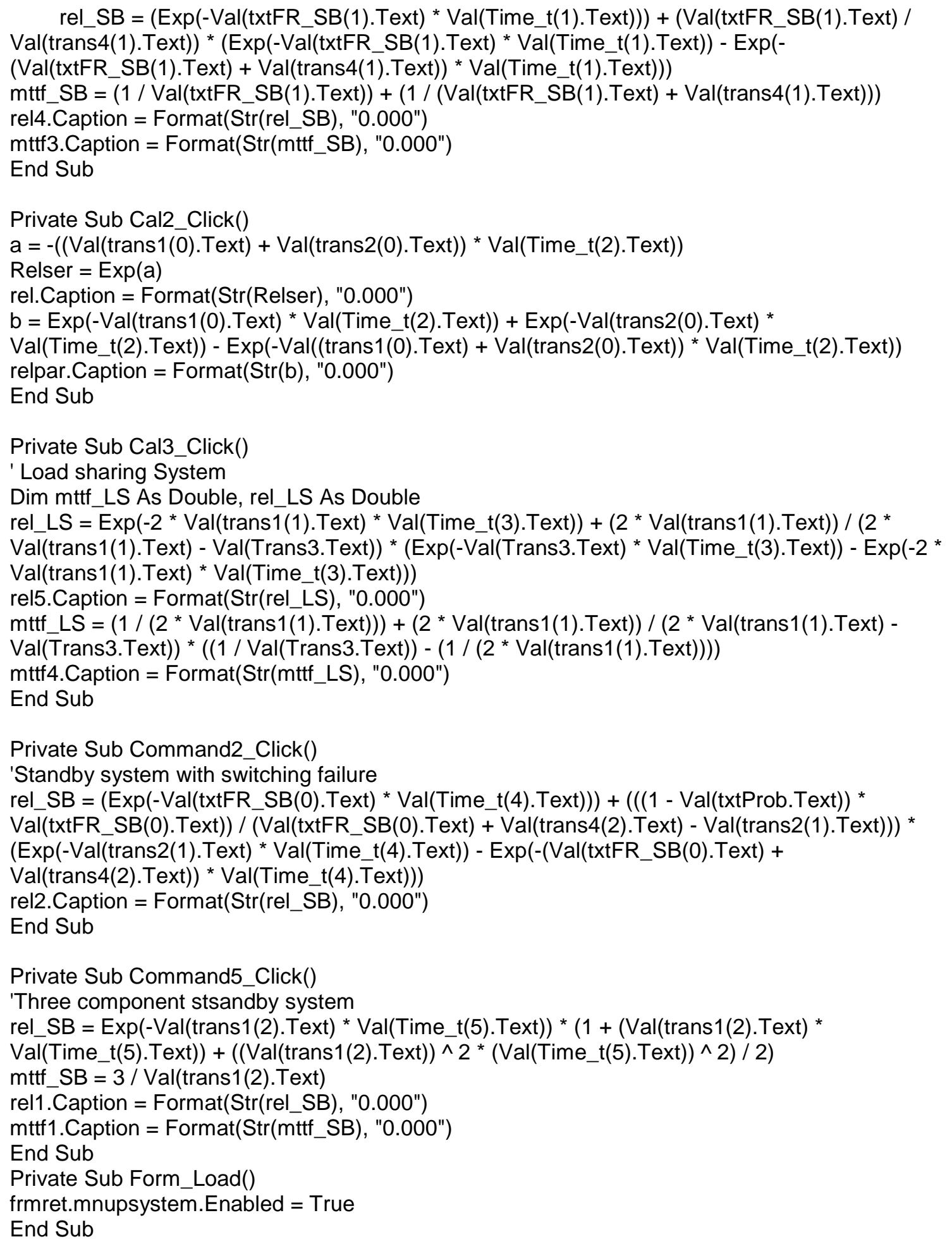

\section{$\underline{\text { Hardware Reliability Form }}$}

Option Explicit

Dim File_Name As String

Private Sub cmdlmport_Click()

On Error GoTo err_handle

Dim i As Single, pathname As String, path As String

Dim Inp1 As String, noofdata As Double, $n$ As Integer 
Dim j As Integer, $\mathrm{k}$ As Integer, I As Integer

Open File_Name For Input As \#1

'calculating number of enteries

$\mathrm{n}=0$

Do While Not EOF(1)

Input \#1, noofdata

$\mathrm{n}=\mathrm{n}+1$

Loop

Close \#1

Open File_Name For Input As \#1

$\mathrm{i}=0$

Do While Not EOF(1)

$i=i+1$

Input \#1, failure(i)

Loop

Close \#1

txtnumberoffailures 1 . Text $=\mathrm{i}$

noOffailures. Text $=\mathrm{i}$

For $\mathrm{i}=1$ To $\mathrm{n}$

gridexpo. $\mathrm{Col}=0$

gridexpo. Row $=\mathrm{i}$

gridexpo. Text $=\mathrm{i}$

gridexpo. $\mathrm{Col}=1$

gridexpo. Row $=\mathrm{i}$

gridexpo. Text $=$ Val(failure $(\mathrm{i}))$

Next i

err_handle:

If $\overline{\mathrm{Err}}=75$ Then

MsgBox "Please Select a File to Open!"

End If

End Sub

Private Sub cmdopen2_Click()

With commondc

.Filter $=$ "Text Files $\left.\left({ }^{*} . \mathrm{txt}\right)\right|^{*}$. txt $\mid "+$ "Hardware Files $\left.\left({ }^{*} . \mathrm{hrd}\right)\right|^{*}$.hrd|" + "Software Files $\left.\left({ }^{*} . \mathrm{st}\right)\right|^{*} . \mathrm{sft} \mid "+$ "All

Files $\left.\left({ }^{*} .{ }^{*}\right)\right|^{*} .{ }^{*} \mid "$

.ShowOpen

File_Name $=$.FileName

End With

End Sub

Private Sub end_Click()

End

End Sub

Private Sub Command1_Click()

Dim p1 As Double

If txtalpha.Text = "' Then

MsgBox "Input the level of significance", vbCritical

Exit Sub

End If

p1 = Val(txtalpha. Text)

If IsNumeric(txtalpha.Text) Then

p1 = Val(txtalpha.Text)

If $\mathrm{p} 1<0$ Or $\mathrm{p} 1>1$ Then

MsgBox " invalid number"

Exit Sub

End If 


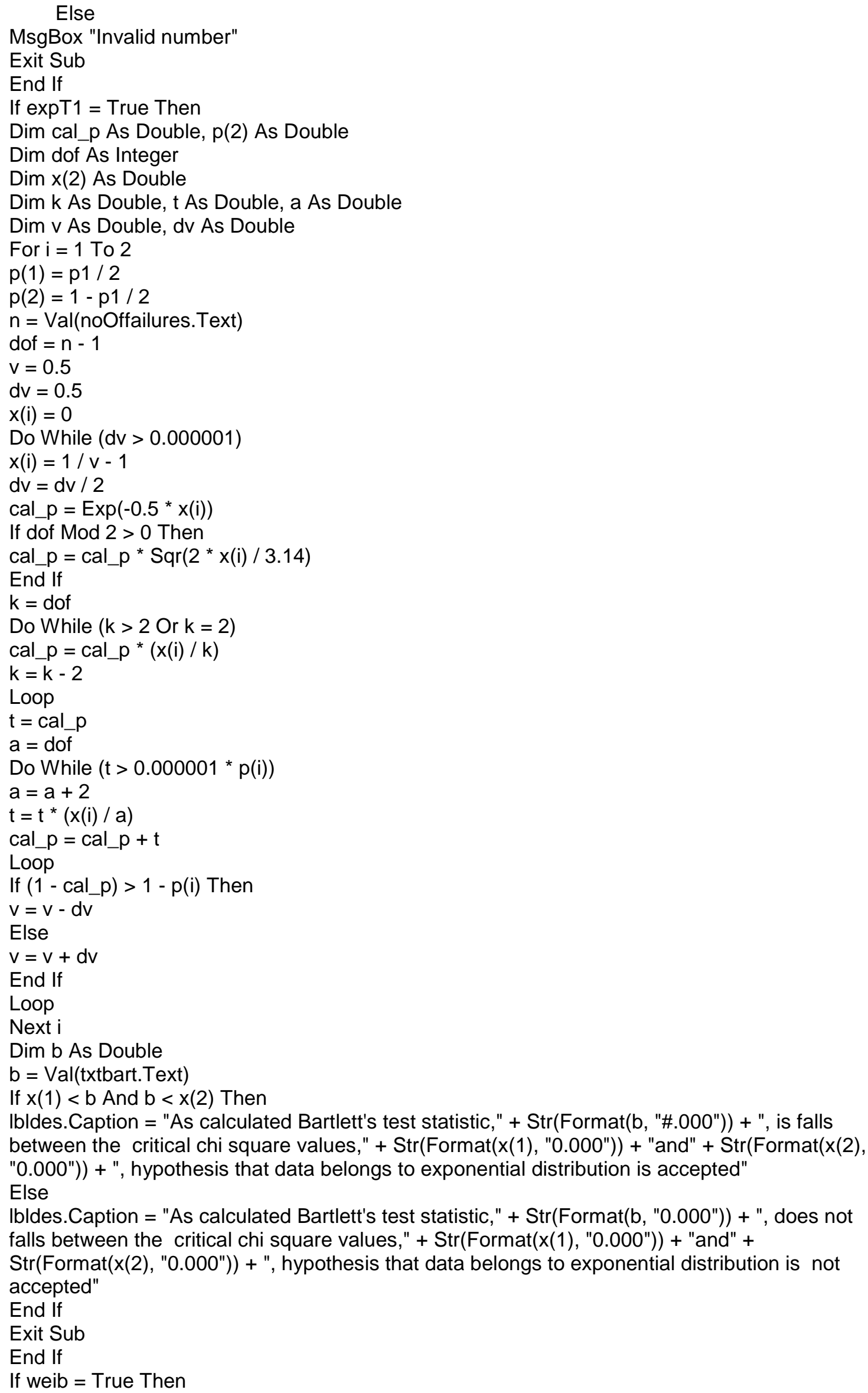


Dim n1 As Double, n2 As Double, $\mathrm{k} 1$ As Double, $\mathrm{k} 2$ As Double, $\mathrm{f}$ As Double

Dim ff As Double

$\mathrm{n}=\mathrm{Val}($ noOffailures. Text)

$\mathrm{k} 1=\operatorname{lnt}(\mathrm{n} / 2)$

$\mathrm{k} 2=\operatorname{lnt}((\mathrm{n}-1) / 2)$

$\mathrm{p} 1=\mathrm{Val}(\mathrm{txtalpha} \cdot \mathrm{Text})$

$\mathrm{n} 1=2{ }^{*} \mathrm{k} 1$

$\mathrm{n} 2=2{ }^{*} \mathrm{k} 2$

$\mathrm{ff}=\operatorname{afishf}(\mathrm{p} 1, \mathrm{n} 1, \mathrm{n} 2)$

If $\mathrm{ff}<$ mann Then

Ibldes.Caption = "As critical F-value," + Str(Format(afishf(p1, n1, n2), "0.000")) + ", is less than

the calculated Mann's test statistic," + Str(Format(mann, "\#.000")) + ", hypothesis that data

belongs to weibull distribution is not accepted"

Else

Ibldes.Caption = "As calculated Mann's test statistic," + Str(Format(mann, "\#.000")) + ", is less

than the critical F-value," + Str(Format(afishf(p1, n1, n2), "0.000")) + ", hypothesis that data

belongs to weibull distribution is accepted"

End If

End If

If NormOpt $=$ True Then

Dim ks2 As Double

$\mathrm{n}=\mathrm{Val}($ noOffailures. Text)

$\mathrm{p} 1=\mathrm{Val}(\mathrm{txtalpha} \cdot \mathrm{Text})$

If $(\mathrm{p} 1=0.2$ Or $\mathrm{p} 1=0.15$ Or $\mathrm{p} 1=0.1$ Or $\mathrm{p} 1=0.05$ Or $\mathrm{p} 1=0.01)$ And $(\mathrm{n}>=4$ Or $\mathrm{n}<=20$ Or $\mathrm{n}=25$

Or $\mathrm{n}=30$ ) Then

If $\mathrm{p} 1=0.2$ Then

If $n>=4$ And $n<=20$ Then

frmks.grdks. Col $=1$

frmks.grdks. Row $=\mathrm{n}-3$

$\mathrm{ks} 2=\mathrm{Val}($ frmks.grdks. Text)

Else

If $n=25$ Then

frmks.grdks. Col $=1$

frmks.grdks. Row $=18$

ks2 $=$ Val(frmks.grdks. Text)

End If

If $n=30$ Then

frmks.grdks. Col $=1$

frmks.grdks. Row $=18$

$\mathrm{ks} 2=\mathrm{Val}($ frmks.grdks. Text)

End If

If $n>30$ Then

ks2 $=0.736 / \operatorname{Sqr}(\mathrm{n})$

End If

End If

End If

If $\mathrm{p} 1=0.15$ Then

If $n>=4$ And $n<=20$ Then

frmks.grdks. Col $=2$

frmks.grdks. Row $=\mathrm{n}-3$

ks2 $=$ Val(frmks.grdks. Text)

Else

If $n=25$ Then

frmks.grdks. Col $=2$

frmks. grdks. Row $=18$

ks2 $=$ Val (frmks.grdks. Text)

End If

If $\mathrm{n}=30$ Then

frmks.grdks. Col $=2$ 
frmks.grdks. Row $=18$

$\mathrm{ks} 2=\mathrm{Val}($ frmks.grdks. Text)

End If

If $\mathrm{n}>30$ Then

ks2 $=0.768 / \operatorname{Sqr}(n)$

End If

End If

End If

If $\mathrm{p} 1=0.1$ Then

If $n>=4$ And $n<=20$ Then

frmks.grdks. Col $=3$

frmks.grdks. Row $=\mathrm{n}-3$

ks2 $=$ Val(frmks.grdks. Text)

Else

If $n=25$ Then

frmks.grdks. Col $=3$

frmks.grdks. Row $=18$

ks2 $=$ Val(frmks.grdks. Text)

End If

If $n=30$ Then

frmks.grdks. Col $=3$

frmks. grdks. Row $=18$

$\mathrm{ks} 2=\mathrm{Val}($ frmks.grdks. Text $)$

End If

If $\mathrm{n}>30$ Then

ks2 $=0.805 / \operatorname{Sqr}(n)$

End If

End If

End If

If $\mathrm{p} 1=0.05$ Then

If $n>=4$ And $n<=20$ Then

frmks.grdks. Col $=4$

frmks. grdks. Row $=n-3$

ks2 $=$ Val(frmks.grdks. Text)

Else

If $\mathrm{n}=25$ Then

frmks.grdks. Col $=4$

frmks.grdks. Row $=18$

ks2 = Val(frmks.grdks. Text)

End If

If $n=30$ Then

frmks.grdks. Col $=4$

frmks. grdks. Row $=18$

ks2 $=$ Val(frmks.grdks. Text)

End If

If $\mathrm{n}>30$ Then

ks2 $=0.886 / \operatorname{Sqr}(n)$

End If

End If

End If

If $p 1=0.01$ Then

If $n>=4$ And $n<=20$ Then

frmks.grdks. Col $=5$

frmks. grdks. Row $=\mathrm{n}-3$

ks2 $=$ Val(frmks.grdks. Text)

Else

If $\mathrm{n}=25$ Then

frmks.grdks. Col $=5$

frmks.grdks. Row $=18$ 
End If

ks2 $=$ Val(frmks.grdks. Text)

If $n=30$ Then

frmks.grdks. Col $=5$

frmks.grdks. Row $=18$

ks2 $=$ Val(frmks.grdks. Text)

End If

If $\mathrm{n}>30$ Then

ks2 $=1.031 / \operatorname{Sqr}(\mathrm{n})$

End If

End If

End If

Else

MsgBox "Input alpha value from $0.2,0.15,0.10,0.05,0.01$ or data not available for $\mathrm{n}=$

21,22,23,24,26,27,28,29"

Exit Sub

End If

If NormOpt $=$ True Then

Dim k_s1 As Double

k_s1 = Val(txtbart.Text)

If $k \_s 1<k s 2$ Then

Ibldes.Caption = "As calculated Komogorov -Smirnov value," + Str(Format(k_s1, "0.000")) + ", is less than the critical Kolmogorov-Smirnov value," + Str(Format(ks2, "\#.000")) + ", hypothesis that data belongs to Normal distribution is accepted"

Else

Ibldes.Caption = "As critical Komogorov -Smirnov value," + Str(Format(ks2, "0.000")) + ", is less

than the calculated Kolmogorov-Smirnov value ," + Str(Format(k_s1, "\#.000")) + ", hypothesis that data belongs to Normal distribution is not accepted"

End If

End If

End If

End Sub

Function fishf( $\mathrm{f}$ As Double, n1 As Double, n2 As Double)

Dim v As Double, dv As Double, k1 As Double, k2 As Double

Dim x As Double

Dim th As Double, a As Double, sth As Double, cth As Double, b As Double, b1 As Double

Dim c As Double, pi As Double, pid2 As Double, k As Double

$\mathrm{pi}=3.14159265358979$

pid2 $=$ pi $/ 2$

$\mathrm{k} 1=\operatorname{lnt}(\mathrm{n} / 2)$

$\mathrm{k} 2=\operatorname{lnt}((\mathrm{n}-1) / 2)$

$\mathrm{n} 1=2 * \mathrm{k} 1$

$\mathrm{n} 2=2{ }^{*} \mathrm{k} 2$

$\mathrm{x}=\mathrm{n} 2 /\left(\mathrm{n} 1{ }^{*} \mathrm{f}+\mathrm{n} 2\right)$

If $n 1 \operatorname{Mod} 2=0$ Then

fishf $=\operatorname{statcom}(1-x, n 2, n 1+n 2-4, n 2-2){ }^{*}\left(x^{\wedge}(n 2 / 2)\right)$

Exit Function

End If

If $\mathrm{n} 2$ Mod $2=0$ Then

fishf $=1-\operatorname{statcom}(x, n 1, n 1+n 2-4, n 1-2)^{*}\left((1-x)^{\wedge}(n 1 / 2)\right)$

Exit Function

End If

th $=\operatorname{Atn}\left(\operatorname{Sqr}\left(n 1{ }^{*} \mathrm{f} / \mathrm{n} 2\right)\right)$

$\mathrm{a}=$ th $/$ pid2

sth $=\operatorname{Sin}($ th $)$

cth $=\operatorname{Cos}($ th)

If $\mathrm{n} 2>1$ Then

$\mathrm{a}=\mathrm{a}+\left(\mathrm{sth}^{*} \mathrm{cth}^{*}\left(\operatorname{statcom}\left(\mathrm{cth}{ }^{*} \mathrm{cth}, 2, \mathrm{n} 2-3,-1\right)\right) /\right.$ pid2 $)$ 


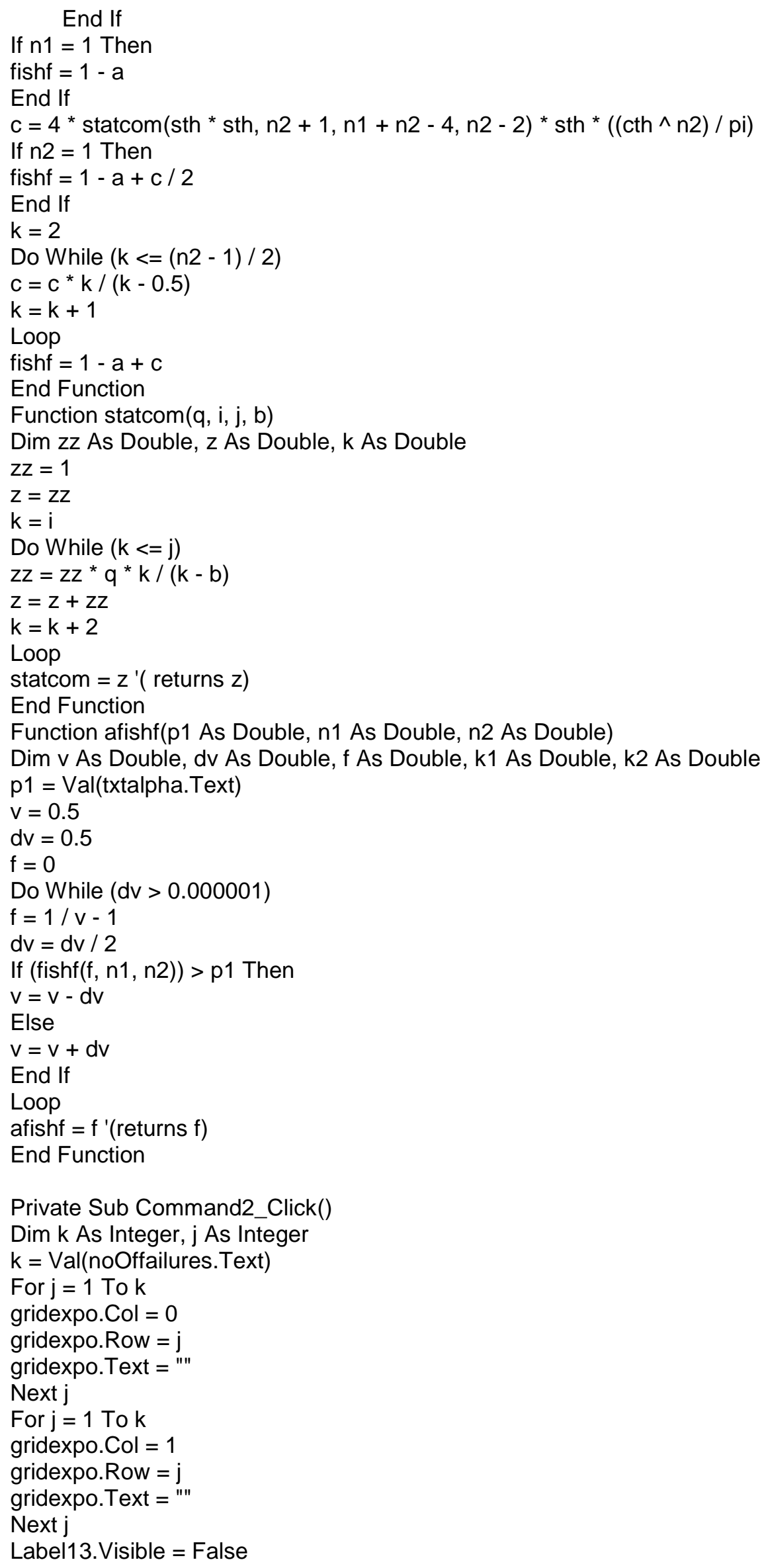




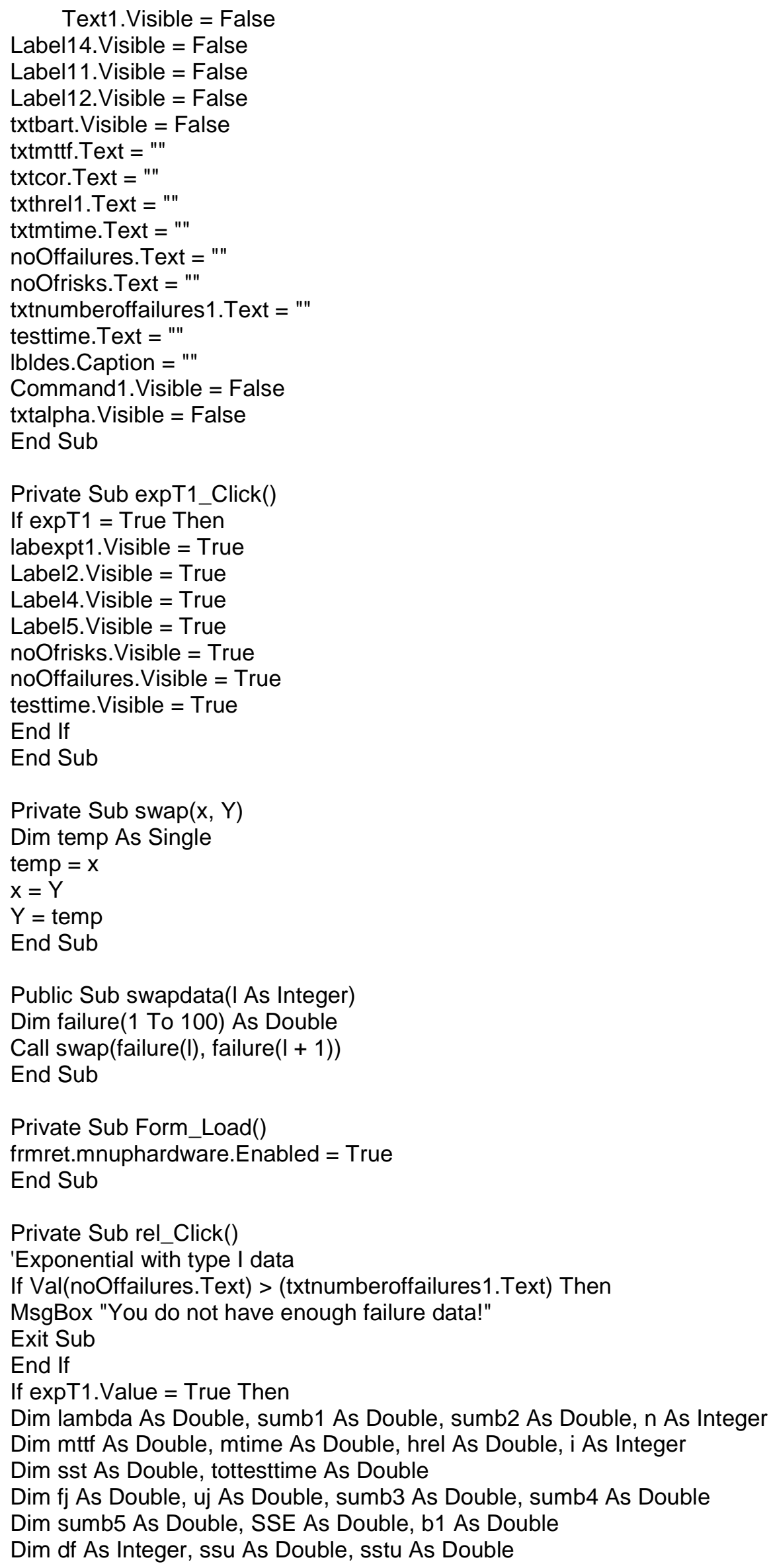


Dim Estderror As Double, cor As Double, TS As Double

Dim b As Double, sumb6 As Double

$\mathrm{n}=\mathrm{Val}$ (noOffailures. Text)

sumb1 $=0$

sumb2 $=0$

sumb3 $=0$

sumb4 $=0$

sumb5 $=0$

sumb6 $=0$

$\mathrm{df}=$ noOffailures. Text -2

For $\mathrm{i}=1$ To $\mathrm{n}$

gridexpo. $\mathrm{Col}=1$

gridexpo. Row $=\mathrm{i}$

sumb1 $=$ sumb1 + gridexpo.Text

sumb2 $=$ sumb2 $+($ gridexpo.Text $) \wedge 2$

$\mathrm{fj}=(\mathrm{i}-0.3) /($ noOfrisks. Text +0.4$)$

$u j=\log (1 /(1-\mathrm{fj}))$

sumb3 = sumb3 + uj

sumb4 $=$ sumb4 $+u^{\wedge} \wedge 2$

sumb5 = sumb5 + gridexpo. Text * $u j$

sumb6 $=$ sumb6 + Log(gridexpo.Text)

Next $\mathrm{i}$

b1 = sumb5 / sumb2

sst $=$ sumb2 $-\left((\text { sumb1 })^{\wedge} 2 /\right.$ noOfrisks. Text $)$

ssu $=$ sumb4 $-(\text { sumb3 })^{\wedge} 2 /$ noOfrisks. Text

sstu = sumb5 - (sumb1 * sumb3) / noOfrisks. Text

SSE $=$ ssu $-\mathrm{b} 1{ }^{*}$ sstu

Estderror $=(\mathrm{SSE} / \mathrm{df}) \wedge 0.5$

cor $=\mathrm{sstu} /\left(\left(\mathrm{sst}^{*} \mathrm{ssu}\right)^{\wedge} 0.5\right)$

$\mathrm{TS}=\mathrm{b} 1{ }^{*}(\mathrm{sst})^{\wedge} 0.5 /$ Estderror

txtcor.Text = Format(Str(cor), "0.000")

'reliability calculation

mtime $=$ Val(txtmtime.Text)

tottesttime $=$ sumb1 + (noOfrisks. Text - noOffailures. Text ${ }^{*}$ testtime.Text

lambda $=\mathrm{Val}($ noOffailures. Text) $/$ tottesttime

$\mathrm{mttf}=1 /$ lambda

txtmttf.Text = Format(Str(mttf), "0.000")

hrel $=\left(\operatorname{Exp}\left(-\right.\right.$ lambda ${ }^{*}$ mtime $\left.)\right)$

If hrel $<0$ Then

txthrel1. Text $=0$

Else

If hrel $>1$ Then

txthrel1. Text $=1$

Else

txthrel1.Text = Format(Str(hrel), "0.000")

End If

End If

'bartletts' test

$b=\left(2\right.$ * noOffailures. Text * $\left(\log \left((1 /\right.\right.$ noOffailures. Text $){ }^{*}$ sumb1 $)-(1 /$ noOffailures.Text $)$ *

sumb6)) / $\left(1+(\right.$ noOffailures. Text +1$) /\left(6{ }^{*}\right.$ noOffailures. Text $\left.)\right)$

If $\operatorname{expT1}$. Value $=$ True Then

Label12. Visible $=$ False

Label15. Visible $=$ False

txtbart.Visible $=$ True

Label11. Visible $=$ True

Label13. Visible $=$ True

Label14. Visible $=$ True

Text1. Visible $=$ True

Label13.Caption = "Distribution selected: Exponential" 
Text1.Text $=$ " Bartletts test value is then compared with the Chisquared value (also known as critical value) with degrees of freedom" + Str(df) + " and level of significance alhpa (user decides this value) from the standard table. If the Bartlett's test value is less than the critical value than the data is good fit for exponential distribution"

txtbart.Text = Format(Str(b), "0.000")

Label14.Caption = "Failure Rate (Lambda) =" + Format(Str(lambda), "0.00000")

txtalpha. Visible $=$ True

Command1. Visible $=$ True

End If

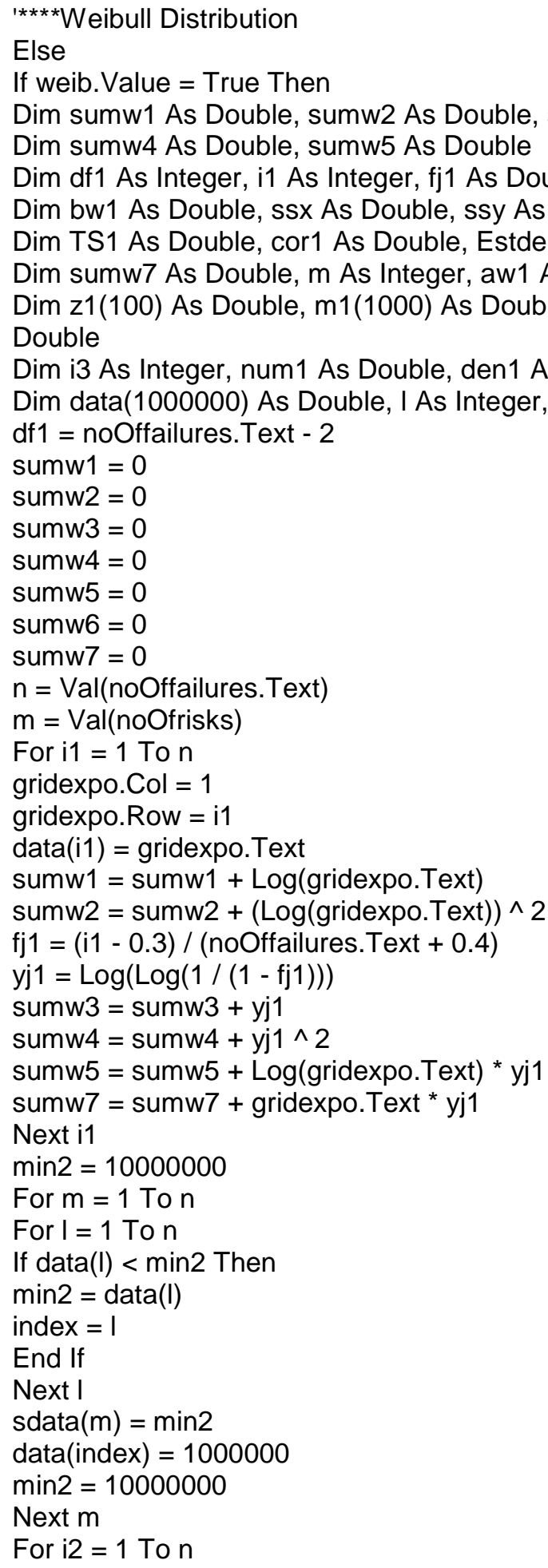




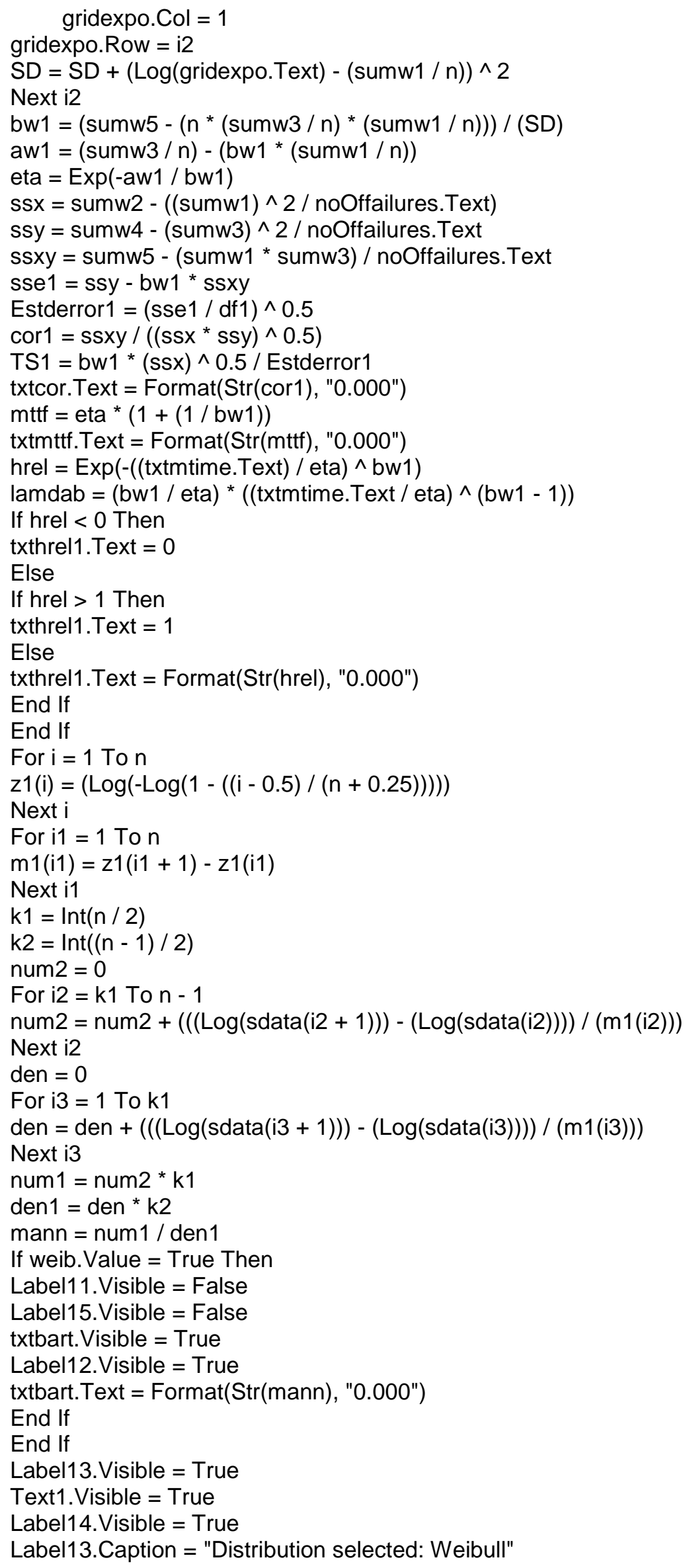


Text1.Text $=$ " Mann test value is then compared with the F- value (also known as critical value) with degrees of freedom" + Str(n-2) + " and level of significance alhpa (user decides this value) from the standard table. If the Mann's test value is less than the critical value than the data is good fit for Weibull distribution"

Label14.Caption = "Failure Rate (Lamda) =" + Format(Str(lamdab), "0.00000")

txtalpha. Visible $=$ True

Command1. Visible $=$ True

End If

${ }^{* * \star * * * *}$ Normal distribution

If NormOpt. Value $=$ True Then

'** to calculate meant time.

Dim dfnormal, no_risk As Integer

Dim sumdata, diff, diffsq, sumsqdiff, sumpro, sumtime, sumnorvalue, sumsqtime, sumsqnormal, be1, be0, estmean, eststd As Double

Dim sst1, ssz, sstz, SSE2, se, TS2, Stdev, correlation, sumnormalvalue As Double

$\mathrm{n}=\mathrm{Val}($ noOffailures. Text)

Dim meantime, sampmean, sampstd As Double

Dim sumg, pnormvalue, diff_tm, diff_gm, ae1, ae2, ae3, p, zek, yek, phik, qek, vek, capk, wek, sumwek, estimean, diff_tem, diff_wm, estistd, reliabili, failrate As Double

Dim norvalues(1000), fej(1000) As Double

sumdata $=0$

For $\mathrm{i}=1$ To $\mathrm{n}$

gridexpo. $\mathrm{Col}=1$

gridexpo. Row $=\mathrm{i}$

sumdata $=$ sumdata + gridexpo.Text

Next i

meantime $=$ sumdata $/ \mathrm{n}$

'**to calculate standard deviation of the data

sumsqdiff $=0$

For $\mathrm{i}=1$ To $\mathrm{n}$

gridexpo. $\mathrm{Col}=1$

gridexpo. Row $=\mathrm{i}$

diff = gridexpo. Text - meantime

diffsq $=$ diff * diff

sumsqdiff $=$ sumsqdiff + diffsq

Next i

Stdev $=$ Sqr (sumsqdiff $/(\mathrm{n}-1))$

${ }^{1 * *}$ to calculate the normal values of the data

For $\mathrm{i}=1$ To $\mathrm{n}$

gridexpo. $\mathrm{Col}=1$

gridexpo. Row $=\mathrm{i}$

$f e j(i)=(i-0.3) /(n+0.4)$

Next $\mathrm{i}$

For $\mathrm{i}=1$ To $\mathrm{n}$

gridexpo. $\mathrm{Col}=1$

gridexpo. Row $=\mathrm{i}$

norvalues $(\mathrm{i})=\left(1 /\left(\operatorname{Sqr}\left(2{ }^{*} 3.14\right)\right)\right){ }^{*} \operatorname{Exp}(-((0.5) *(\mathrm{fej}(\mathrm{i}) \wedge 2)))$

Next i

'** To calculate the slope b1 and intercept b0

sumpro $=0$

sumtime $=0$

sumnorvalue $=0$

sumsqtime $=0$

sumsqnormal $=0$

For $\mathrm{i}=1$ To $\mathrm{n}$

gridexpo. $\mathrm{Col}=1$

gridexpo. Row $=\mathrm{i}$

sumpro $=$ sumpro $+\left(\right.$ gridexpo.Text ${ }^{*}$ norvalues $\left.(i)\right)$ 
sumtime $=$ sumtime + gridexpo. Text

sumnorvalue $=$ sumnorvalue + norvalues $(i)$

sumsqtime $=$ sumsqtime $+\left(\right.$ gridexpo.Text $\left.{ }^{\wedge} 2\right)$

sumsqnormal $=$ sumsqnormal $+\left(\right.$ norvalues $\left.(i)^{\wedge} 2\right)$

Next i

be1 $=\left(\left(n{ }^{*}\right.\right.$ sumpro $)-($ sumtime * sumnorvalue $\left.)\right) /\left((n\right.$ * sumsqtime $)-\left(\left(\right.\right.$ sumtime $\left.\left.\left.{ }^{\wedge} 2\right)\right)\right)$

be $0=($ sumnorvalue $/ \mathrm{n})$ - (be $1{ }^{*}$ sumtime $\left./ \mathrm{n}\right)$

${ }^{1 * *}$ to calculate estimated mean and standard deviation

estmean $=$ be $0 /$ be 1

eststd $=1 /$ be 1

1** degrees of freedom

dfnormal $=$ Val(noOffailures. Text) -2

'** sum of squares of $t$

sst1 $=$ sumsqtime $-(($ sumtime * sumtime $) / n)$

'** sum of squares of $z$

ssz = sumsqnormal - ((sumnorvalue ${ }^{*}$ sumnorvalue $\left.) / n\right)$

'** sum of squares of $t z$

sstz = sumpro - (sumtime * sumnorvalue / $\mathrm{n}$ )

'** sum of squares of error

SSE2 = Ssz - (be1 * sstz)

'** estimated standard error

se $=$ Sqr $($ SSE2 $/ \mathrm{n})$

'** correlation

correlation $=\mathrm{sstz} / \mathrm{Sqr}\left(\mathrm{sst} 1{ }^{*} \mathrm{ssz}\right)$

txtcor . Text $=$ correlation

"** test statistic of $t$ (for slope)

TS2 = be1 / (se / Sqr(sst1))

"** to calculate the sample mean

no_risk $=$ Val $($ noOfrisks. Text $)$

sumg $=($ no_risk $-\mathrm{n}){ }^{*} \mathrm{Val}($ testtime.Text $)$

sampmean $=($ sumtime $+($ sumg $)) /$ no_risk

${ }^{1 * *}$ to calculate the sample standard deviation

diff_tm $=0$

For $\bar{i}=1$ To $n$

gridexpo. Col $=1$

gridexpo. Row $=\mathrm{i}$

diff_tm $=$ diff_tm $+\left((\text { gridexpo.Text }- \text { sampmean })^{\wedge} 2\right)$

Next i

diff_gm $=0$

For $\mathrm{i}=1$ To (no risk - $\mathrm{n}$ )

diff_gm $=$ diff_gm $\left.+((\text { Val(testtime.Text })-\text { sampmean })^{\wedge} 2\right)$

Next i

sampstd $=\operatorname{Sqr}(($ diff_tm + diff_gm $) /($ no_risk -1$))$

$\mathrm{ae} 1=0.4361836$

ae2 $=-0.1201676$

ae3 $=0.937298$

$\mathrm{p}=0.33267$

zek $=($ Val(testtime.Text $)-$ sampmean $) /$ sampstd

yek $=1 /(1+((p *$ zek $)))$

phik $=\left(1 /\left(\operatorname{Sqr}\left(2{ }^{*} 3.14\right)\right)\right){ }^{*} \operatorname{Exp}\left((-0.5) *\left(z e{ }^{\wedge}{ }^{\star} 2\right)\right)$

qek $=$ phik $^{*}\left(\left(a e 1^{*}\right.\right.$ yek $)+\left(a e{ }^{*}\left(\right.\right.$ yek $\left.\left.^{\wedge} 2\right)\right)+\left(a e{ }^{*}\left(\right.\right.$ yek $\left.\left.\left.^{\wedge} 3\right)\right)\right)$

vek $=$ phik $/$ qek

capk $=$ vek ${ }^{*}($ vek - zek $)$

wek $=$ sampmean $+\left(\right.$ sampstd ${ }^{*}$ vek $)$

${ }^{1 * * *}$ to calculate estimated mean

sumwek $=0$

For $\mathrm{i}=1$ To (no_risk $-\mathrm{n}$ )

sumwek = sumwek + wek

Next i 


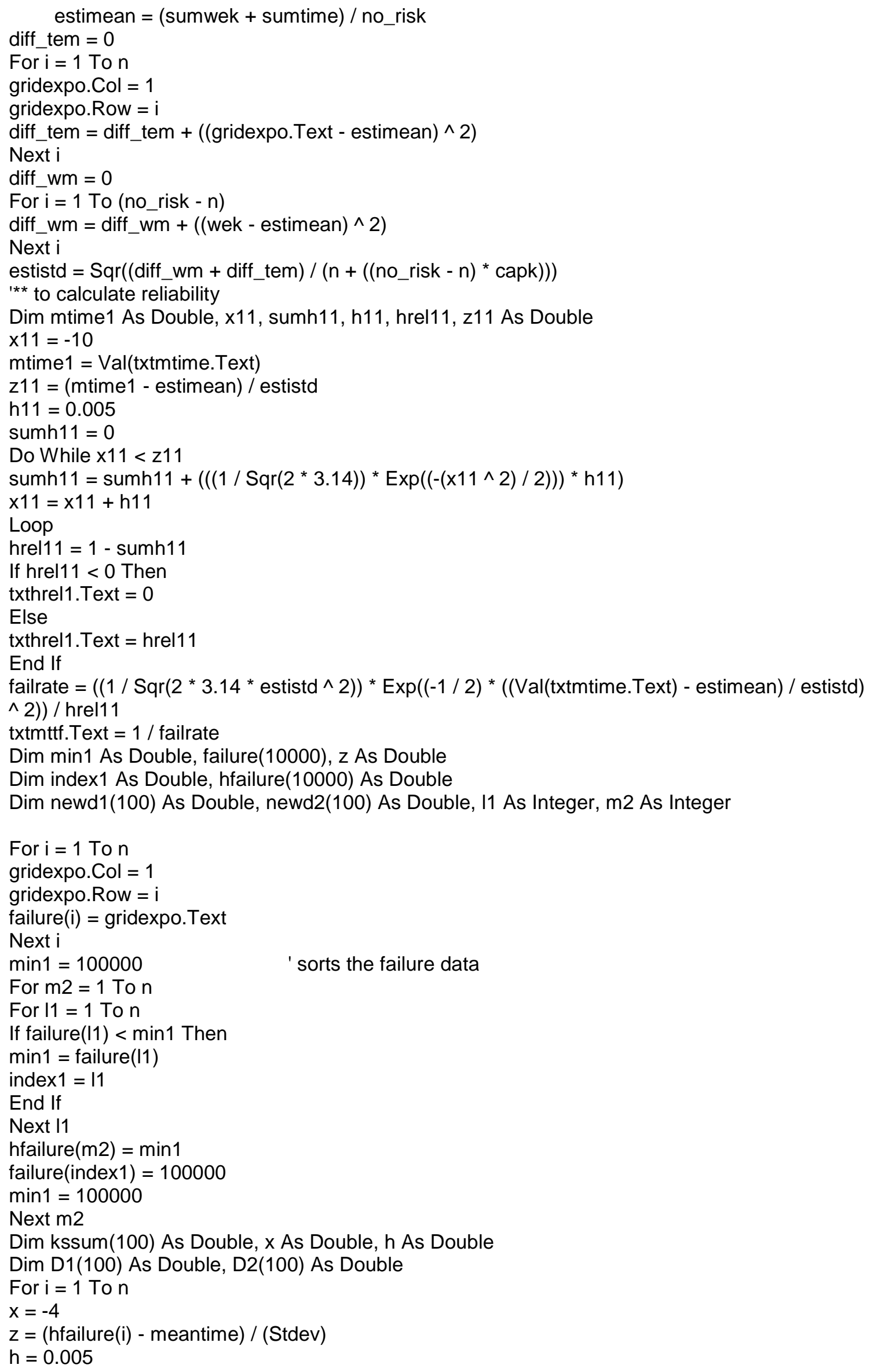




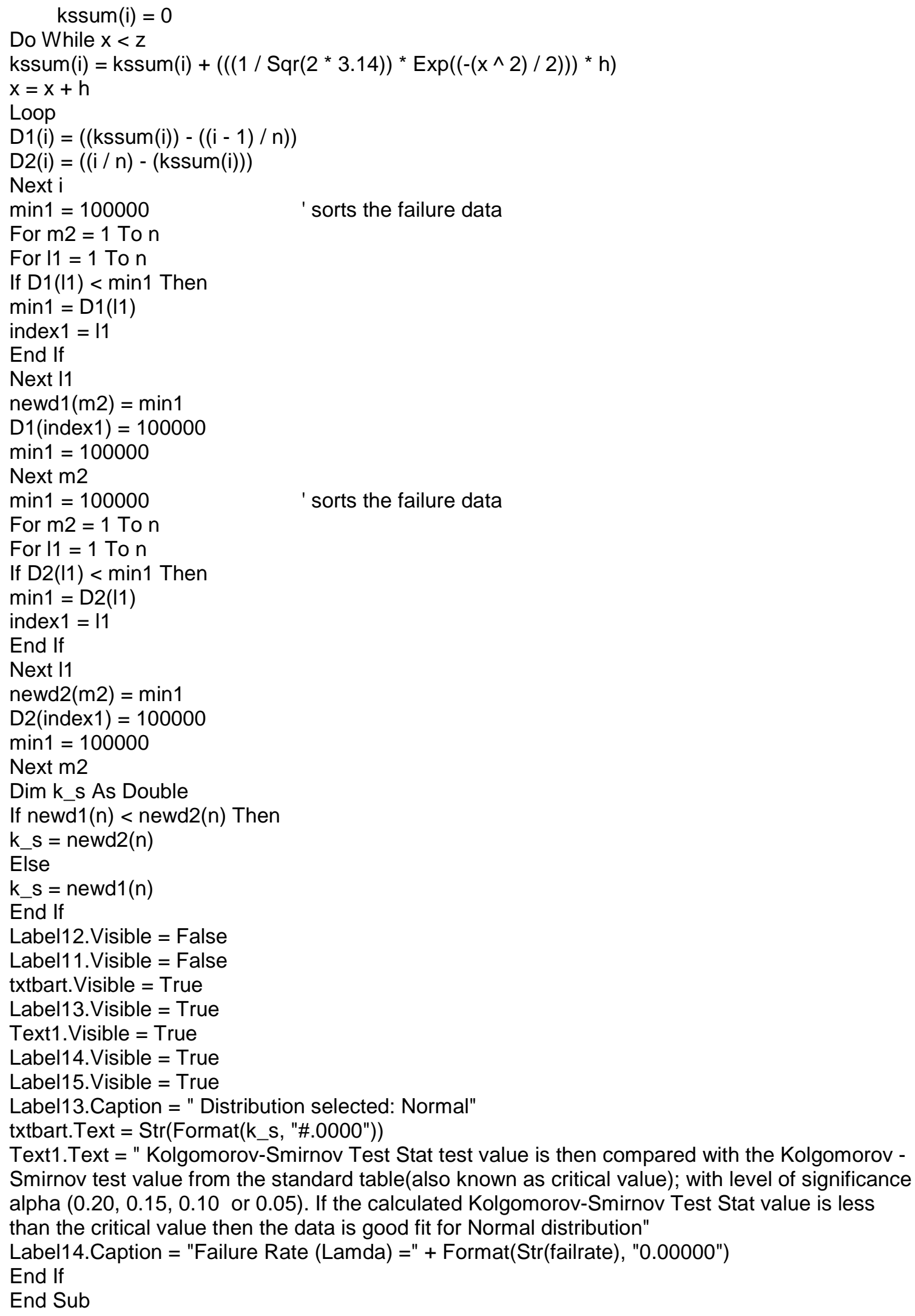

\section{Software Reliability Form}

'ADDITIONAL TIME CALCULATION

$1 * * * * * * * * * * * * * * * * * * * * * * * * * * * *$

Private Sub cmdadditional_Click() 


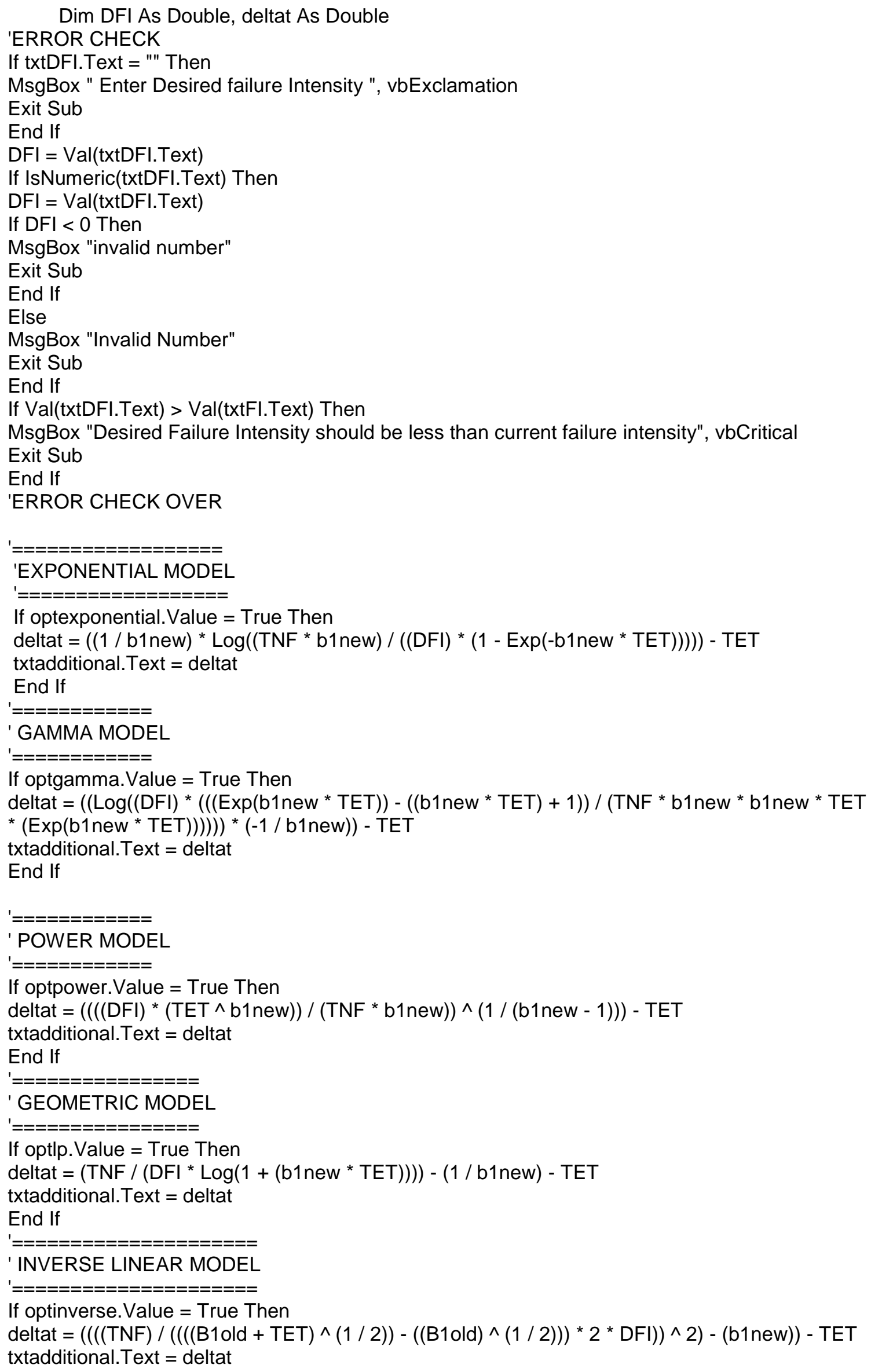




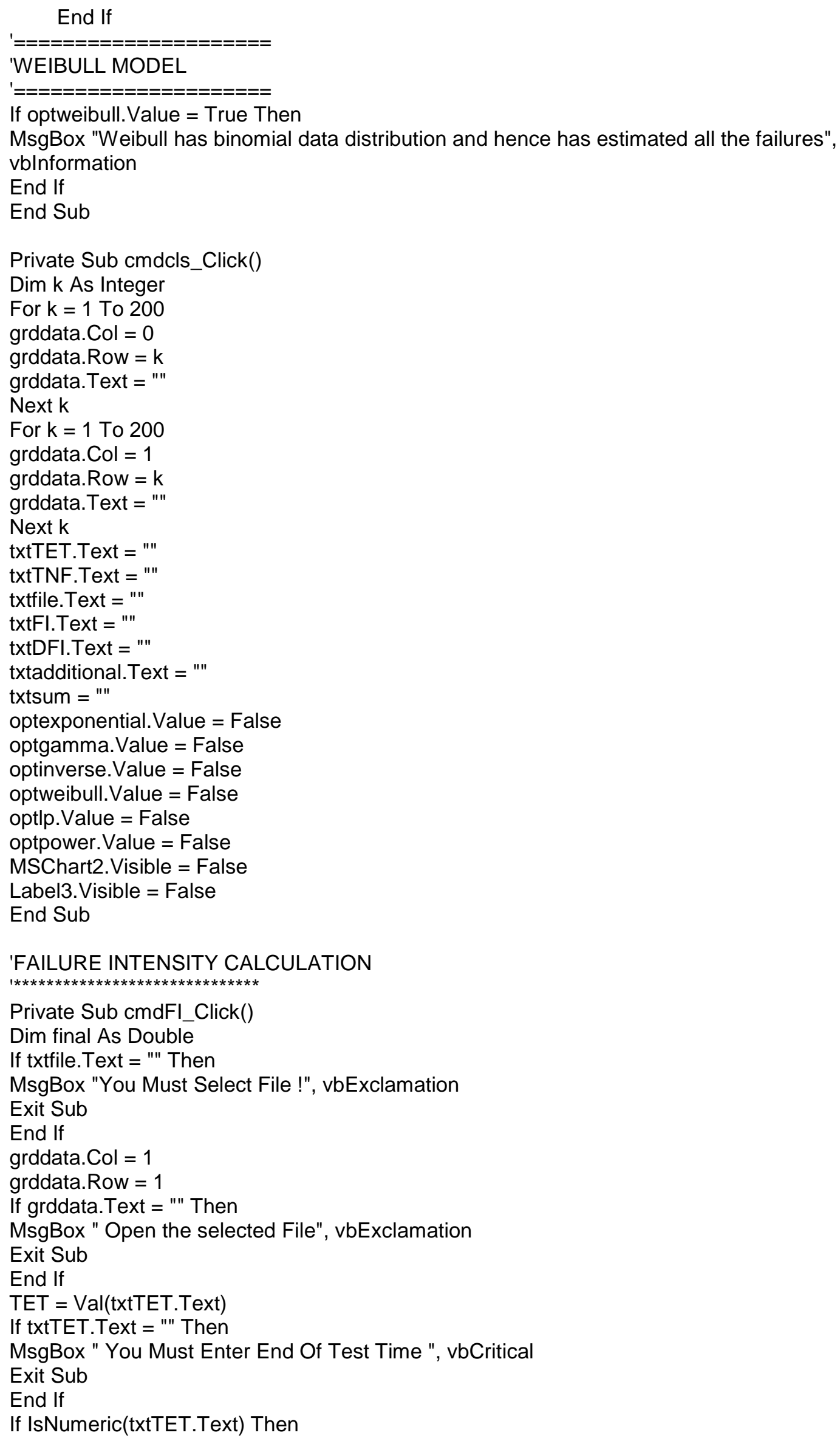




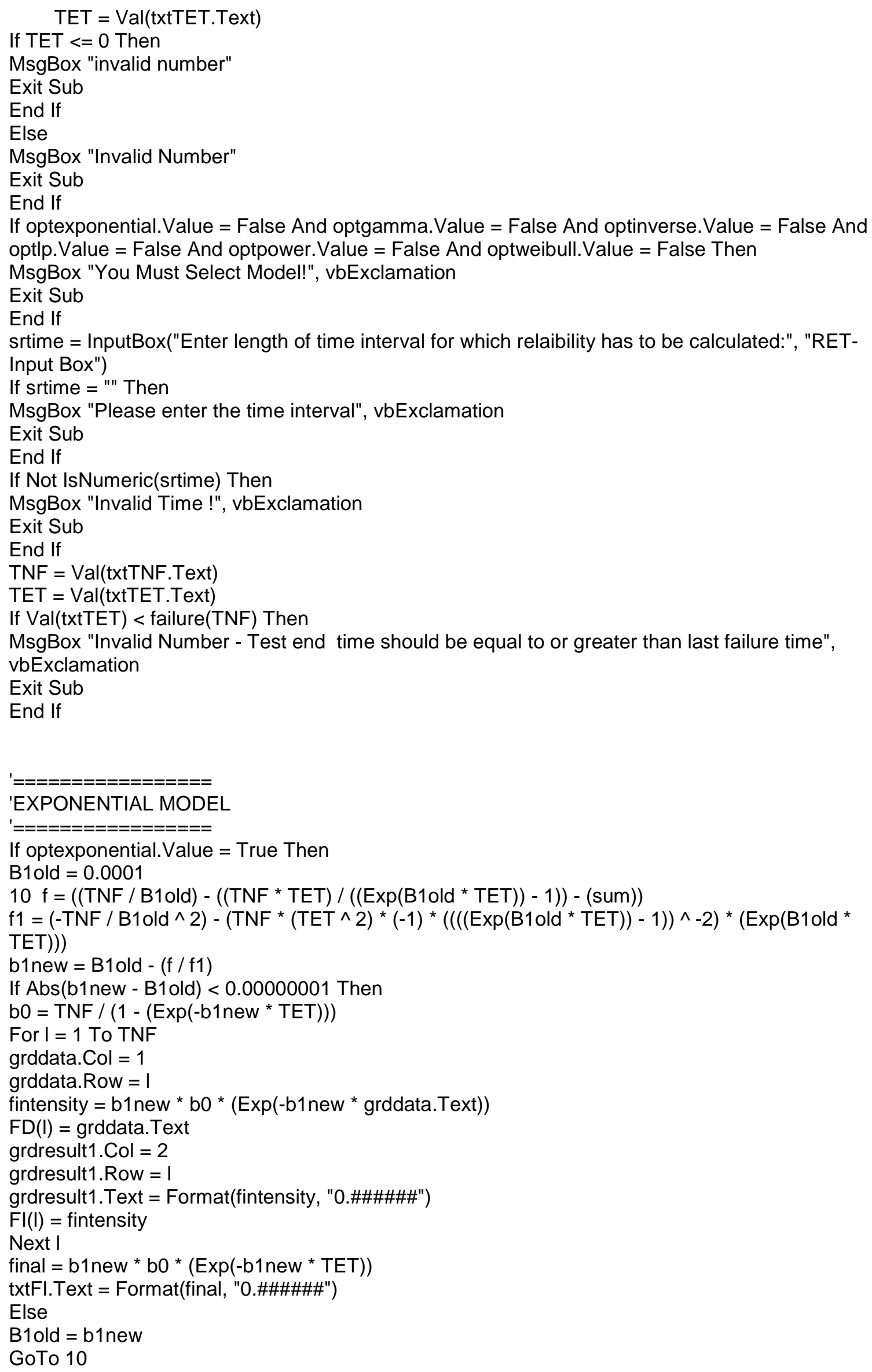




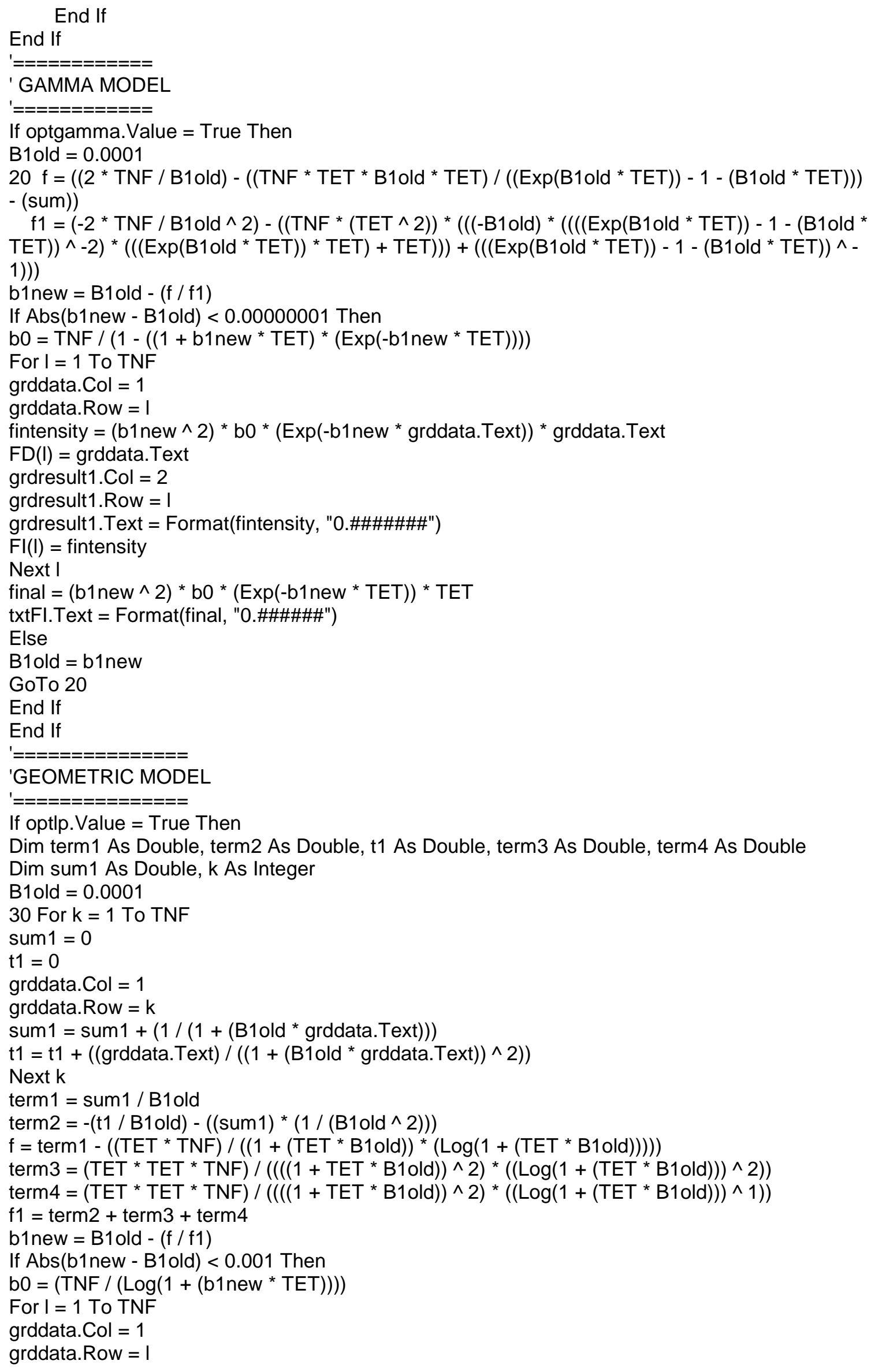




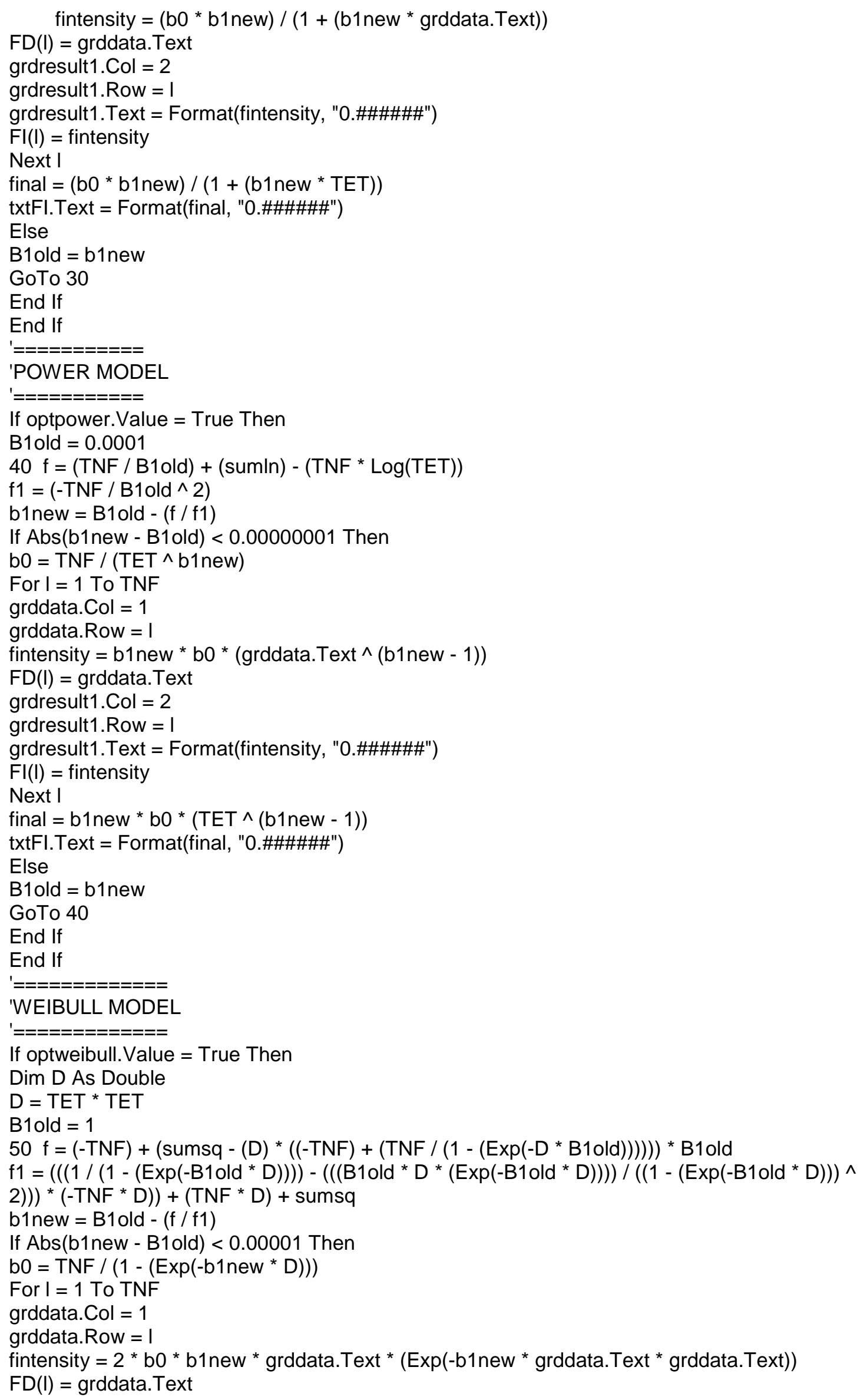




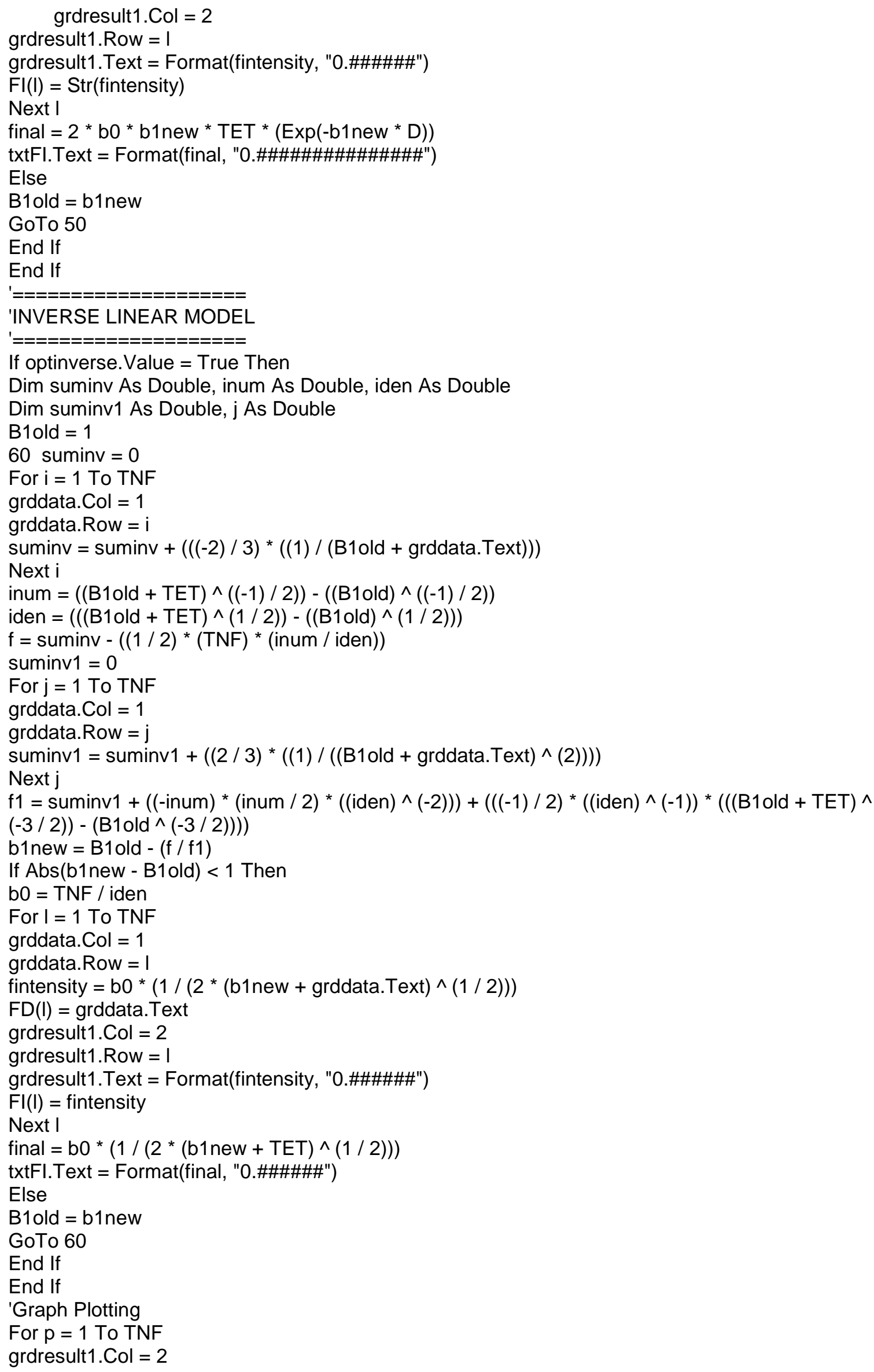




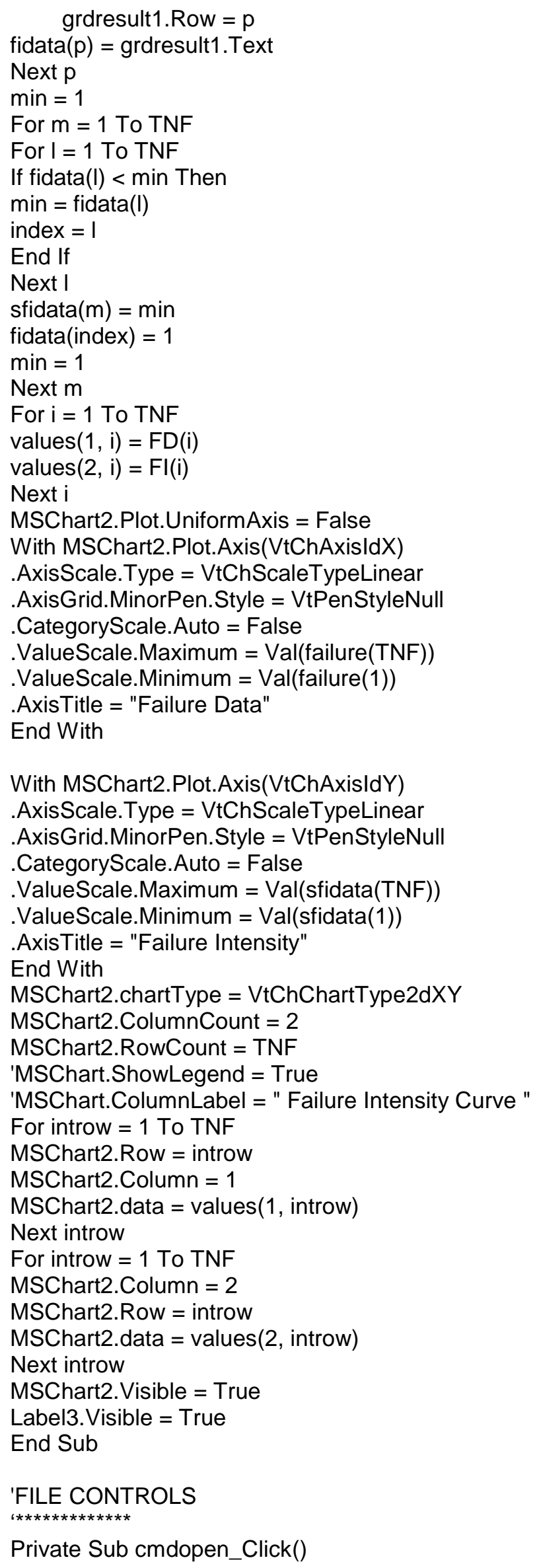




\section{If txtfile. Text = "'" Then}

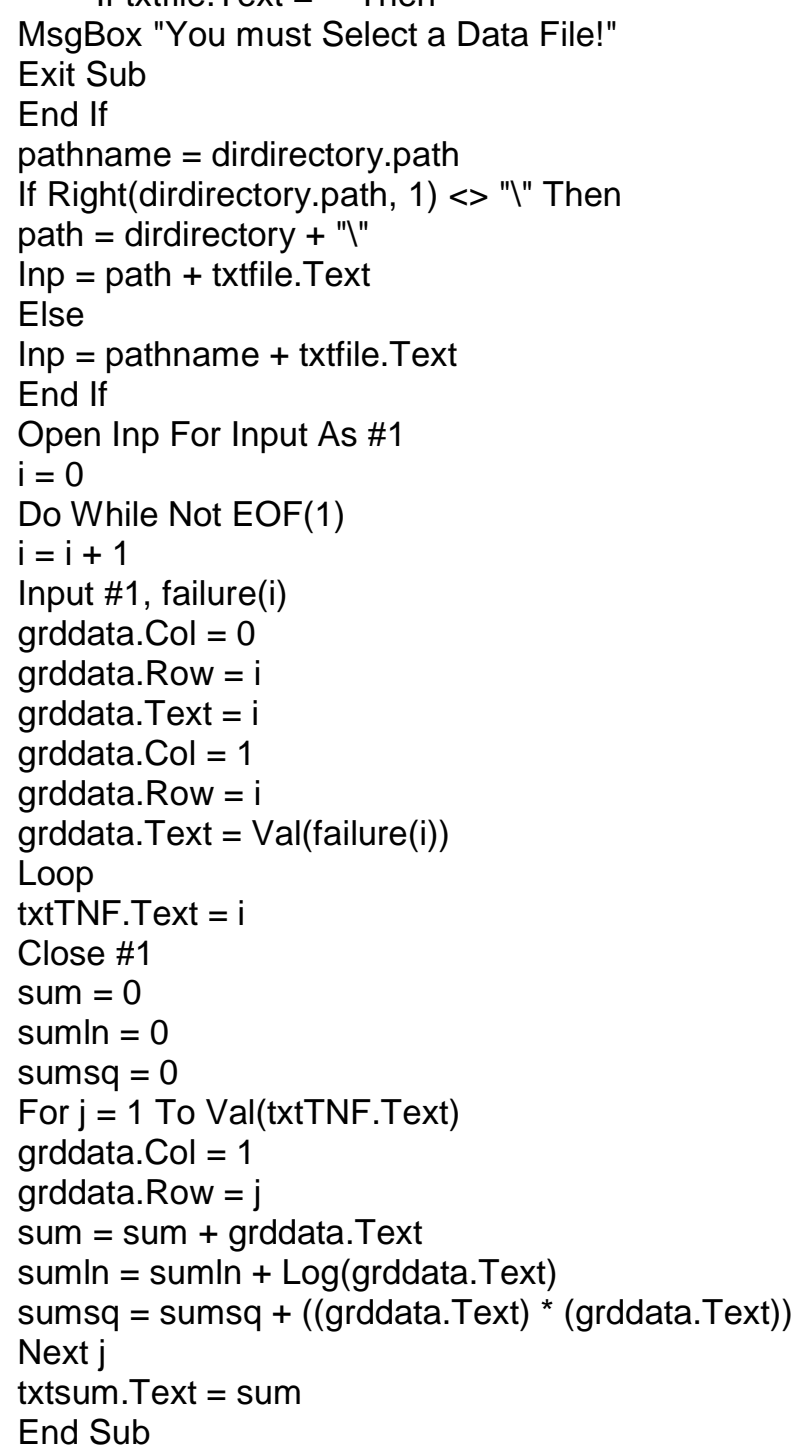

\section{'RESULT TABULATION}

'RESULT TABULA

Private Sub cmdresults_Click()

Dim b As Integer, c As Integer

Open Inp For Input As \#4

$\mathrm{i}=0$

Do While Not EOF(4)

$\mathrm{i}=\mathrm{i}+1$

Input \#4, failure(i)

grdresult1.Col $=0$

grdresult1. Row $=\mathrm{i}$

grdresult1. Text $=\mathrm{i}$

grdresult1.Col $=1$

grdresult1. Row $=\mathrm{i}$

grdresult1.Text $=$ Val(failure $(i))$

Loop

Close \#4

For $\mathrm{C}=1$ To 5

frmresult.grdresult.ColWidth $(\mathrm{c})=1200$

Next c

frmresult.grdresult.Row $=0$ 


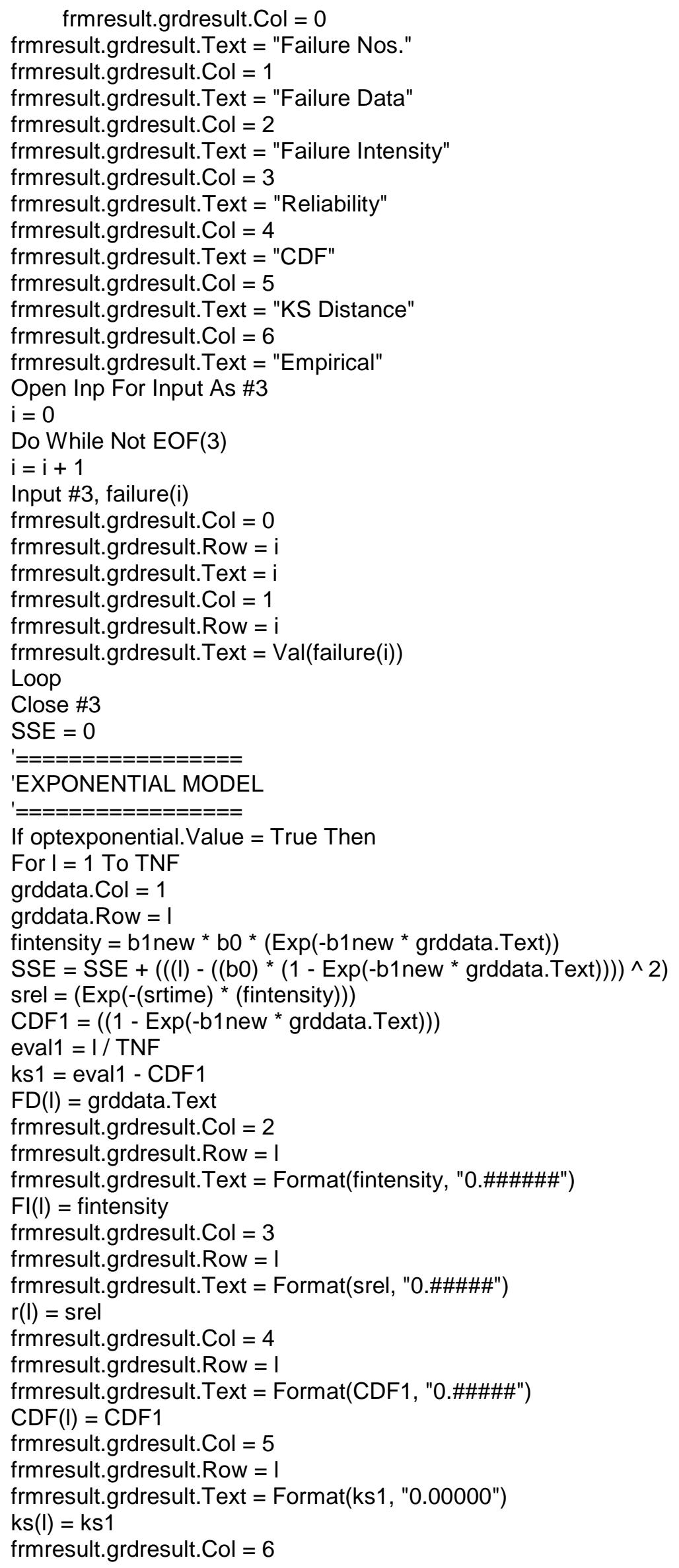


frmresult.grdresult.Row $=1$

frmresult.grdresult.Text = Format(eval1, "0.00000")

eval $(I)=$ eval1

Next I

End If

'============

' GAMMA MODEL

'============

If optgamma. Value $=$ True Then

For I = 1 To TNF

grddata.Col $=1$

grddata. Row $=1$

fintensity $=\left(b 1\right.$ new $\left.{ }^{\wedge} 2\right){ }^{*}$ b0 * $(\operatorname{Exp}(-b 1$ new * grddata.Text $))$ * grddata.Text

srel $=(\operatorname{Exp}(-($ srtime $) *($ fintensity $)))$

SSE $=$ SSE $+\left(\left((I)-\left((b 0) *\left((1)-\left(\left(1+\text { b1 new }{ }^{*} \text { grddata.Text }\right) *\left(E x p\left(-b 1 \text { new }{ }^{*} \text { grddata.Text }\right)\right)\right)\right)\right)\right)^{\wedge}\right.$ 2)

CDF1 $=\left((1)-\left((1+\right.\right.$ b1new * grddata.Text $){ }^{*}(\operatorname{Exp}(-b 1$ new * grddata.Text $\left.\left.))\right)\right)$

eval1 $=\mathrm{I} / \mathrm{TNF}$

$\mathrm{ks} 1=(\mathrm{I} / \mathrm{TNF})-\mathrm{CDF} 1$

$\mathrm{FD}(\mathrm{l})=$ grddata. Text

frmresult.grdresult.Col $=2$

frmresult.grdresult.Row $=1$

frmresult.grdresult.Text = Format(fintensity, "0.\#\#\#\#\#\#")

$\mathrm{Fl}(\mathrm{I})=$ fintensity

frmresult.grdresult.Col $=3$

frmresult.grdresult.Row $=1$

frmresult.grdresult.Text $=$ Format(srel, "0.00000")

$r(I)=$ srel

frmresult.grdresult.Col $=4$

frmresult.grdresult. Row $=1$

frmresult.grdresult.Text = Format(CDF1, "0.\#\#\#\#")

$\mathrm{CDF}(\mathrm{I})=\mathrm{CDF} 1$

frmresult.grdresult.Col $=5$

frmresult.grdresult.Row $=1$

frmresult.grdresult.Text $=$ Format(ks1, "0.00000")

$\mathrm{ks}(\mathrm{I})=\mathrm{ks} 1$

frmresult.grdresult.Col $=6$

frmresult.grdresult.Row $=1$

frmresult.grdresult.Text = Format(eval1, "0.00000")

eval $(I)=$ eval1

Next I

End If

'===============

'GEOMETRIC MODEL

$============$

If optlp. Value $=$ True Then

For I $=1$ To TNF

grddata.Col $=1$

grddata. Row $=1$

fintensity $=\left(\mathrm{b} 0{ }^{*} \mathrm{~b} 1\right.$ new $) /(1+(\mathrm{b} 1$ new * grddata. Text $))$

srel $=(\operatorname{Exp}(-($ srtime $) *($ fintensity $)))$

SSE $=\operatorname{SSE}+\left(\left((\mathrm{I})-\left(\mathrm{b} 0^{*}\left(\log \left(1+\mathrm{b} 1\right.\right.\right.\right.\right.$ new ${ }^{*}$ grddata.Text $\left.\left.\left.\left.)\right)\right)\right) \wedge 2\right)$

$\operatorname{CDF} 1=\left(\log \left(1+b 1\right.\right.$ new ${ }^{*}$ grddata.Text $) / \log \left(1+b 1\right.$ new ${ }^{*}$ failure $\left.\left.(T N F)\right)\right)$

eval $1=\mathrm{I} / \mathrm{TNF}$

$\mathrm{ks} 1=(\mathrm{l} / \mathrm{TNF})-\mathrm{CDF} 1$

$\mathrm{FD}(\mathrm{I})=$ grddata. Text

frmresult.grdresult.Col $=2$

frmresult.grdresult. Row $=1$

frmresult.grdresult.Text = Format(fintensity, "0.\#\#\#\#\#") 


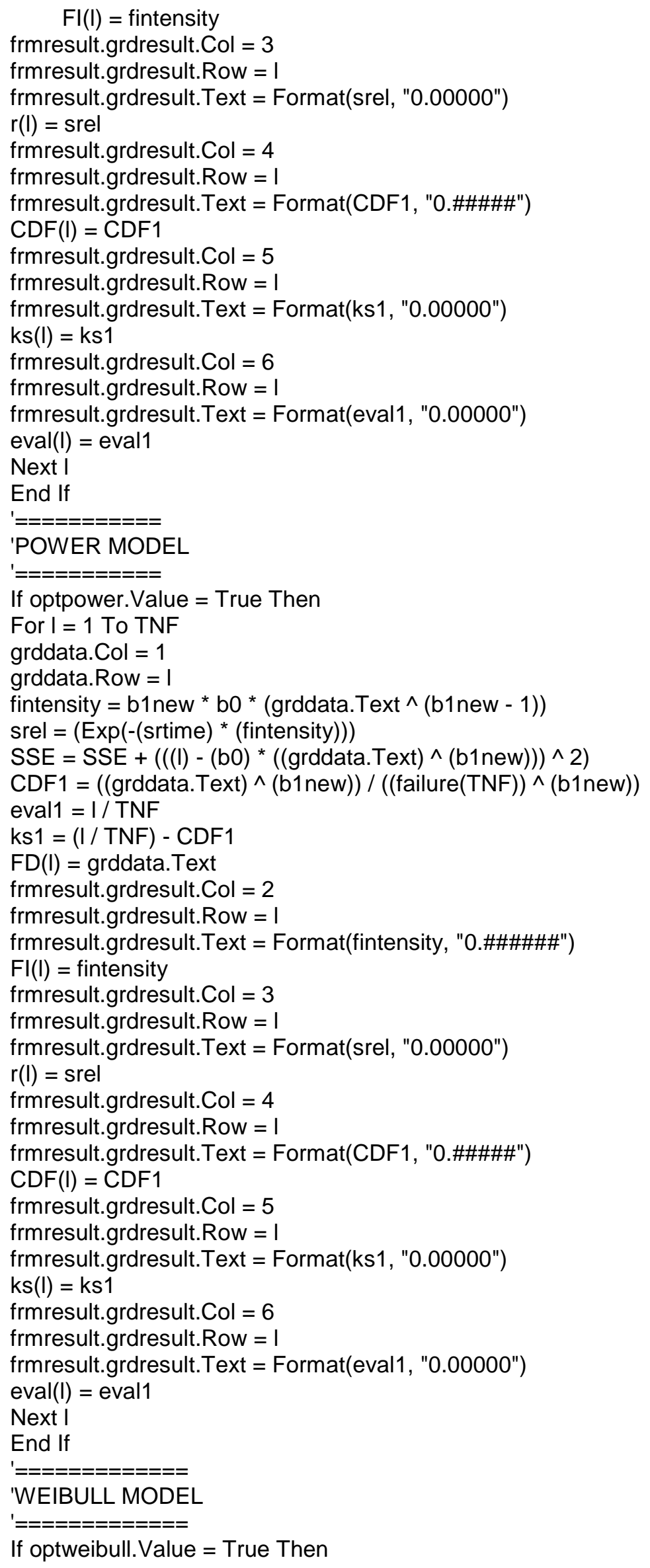




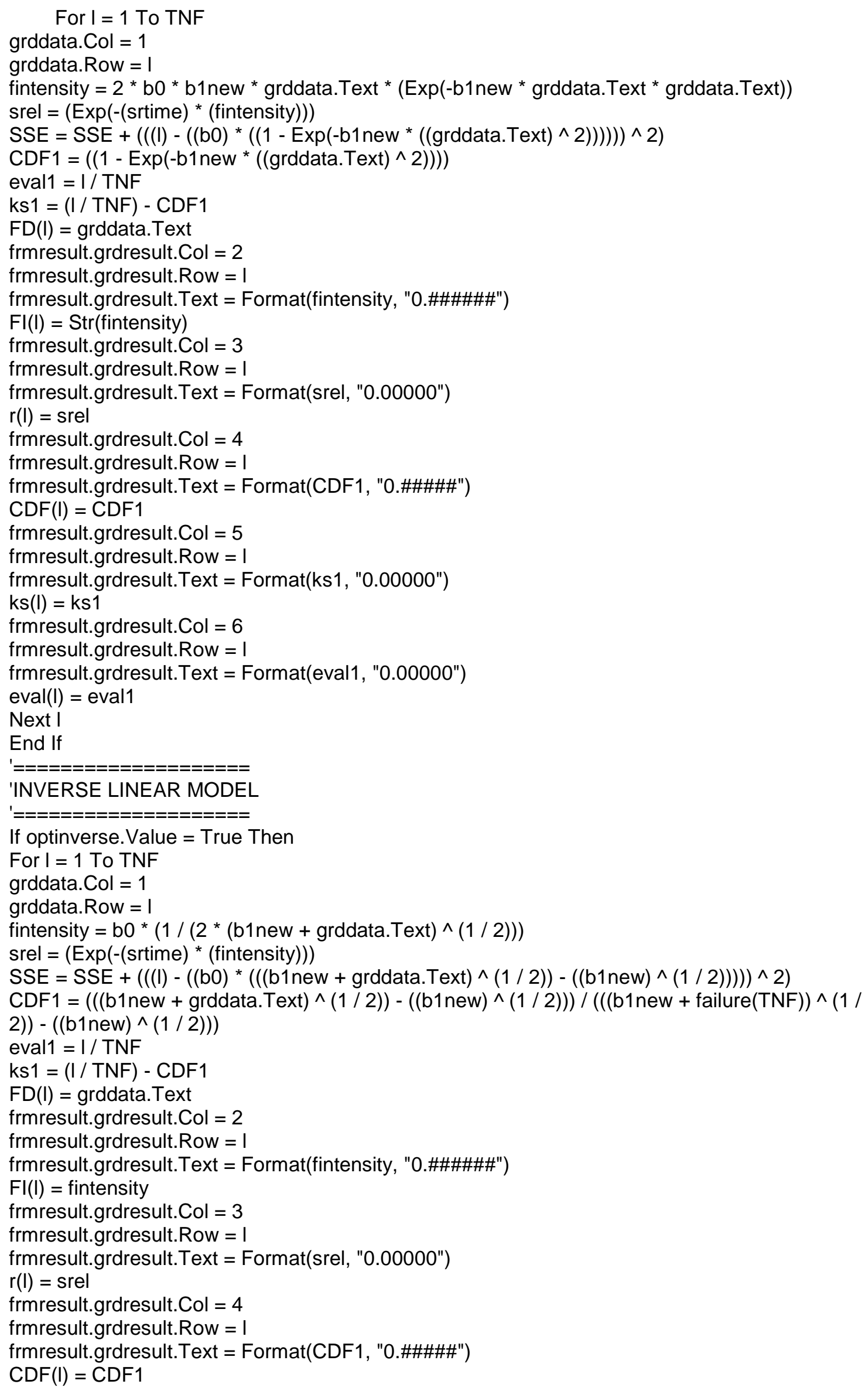




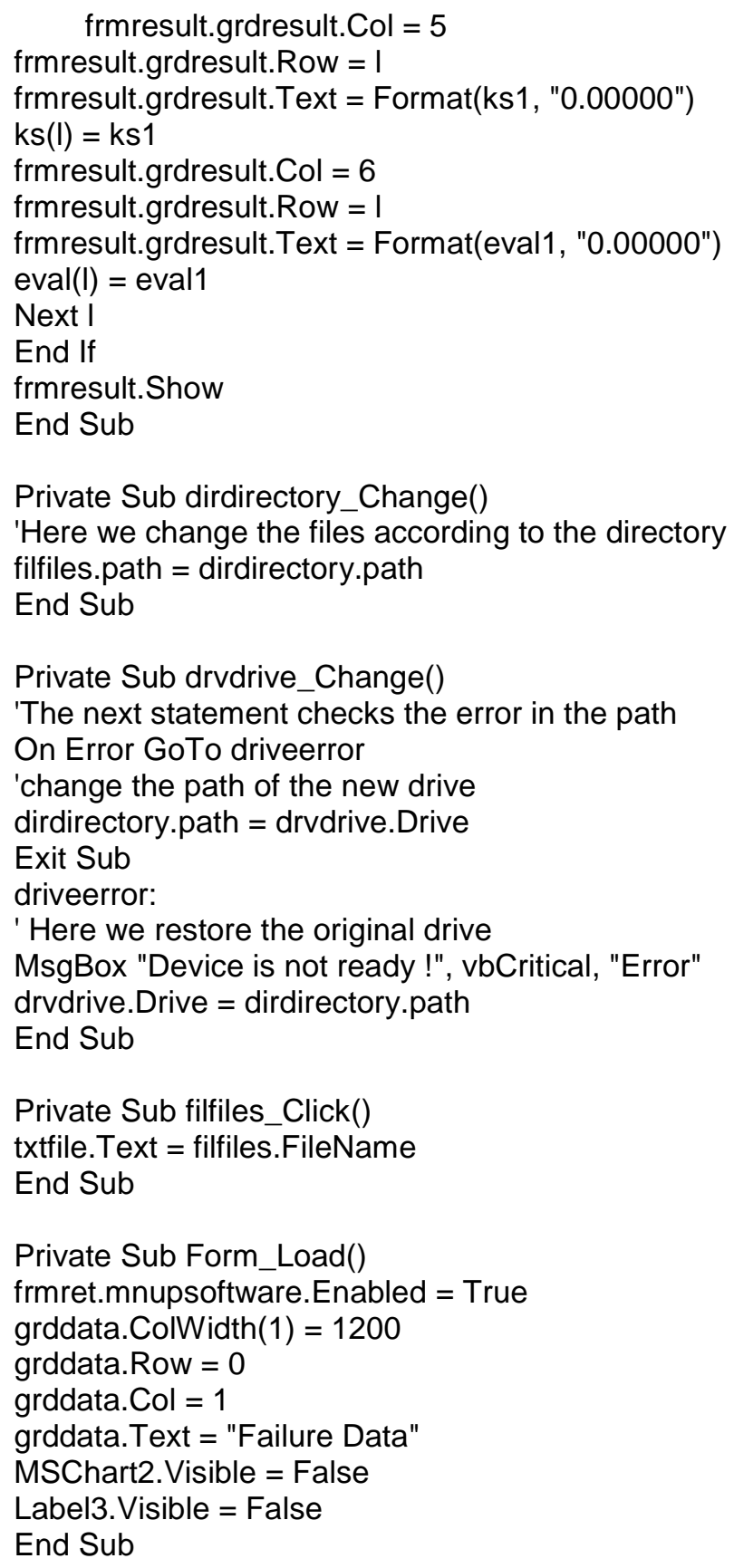

\section{Reliability Calculator}

Option Explicit

Dim frate As Single

Dim pi As Single

Dim x As Double, z As Double

Dim $h$ As Single

Dim sum As Double

Dim nmean As Double, nstddev As Double

Dim Inshape As Double, Inloc As Double

Dim wscale As Single

Dim wshape As Single

Private Sub cmde_Click() 


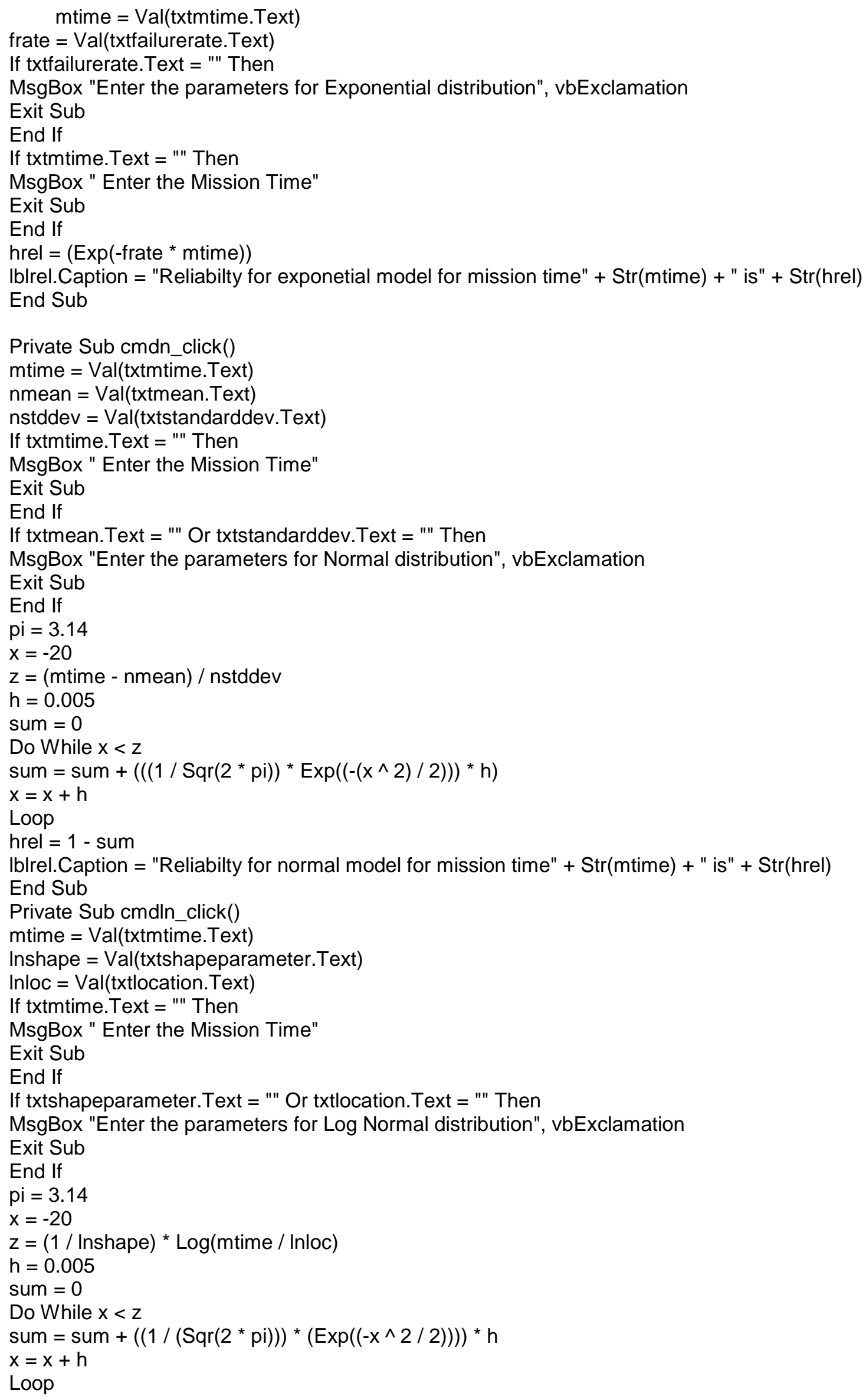




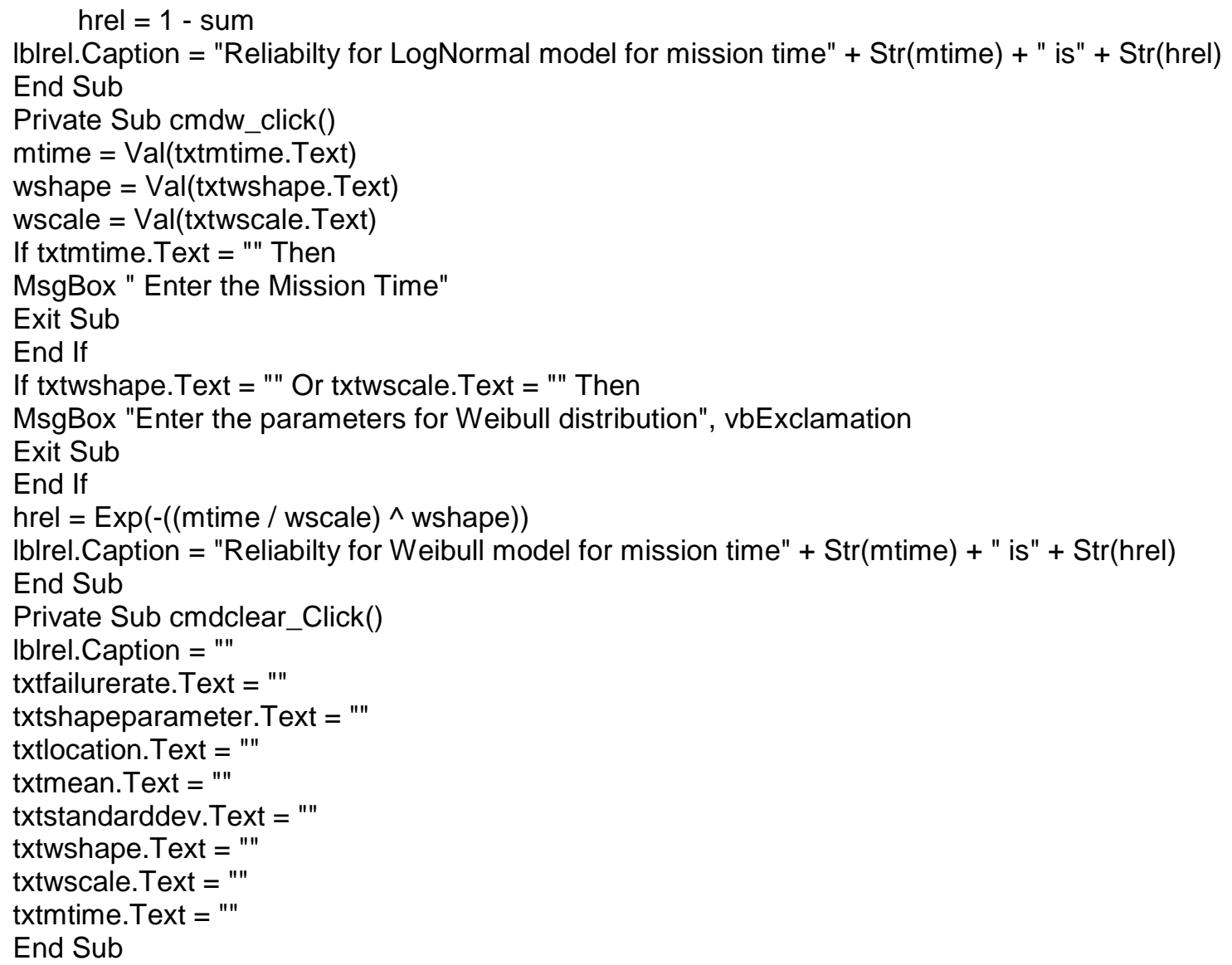

\section{F-Value Calculator}

Option Explicit

Private Sub Command1_Click()

Dim p1 As Double, n1 As Double, n2 As Double

Iblans.Caption = Format(Str(afishf(p1, n1, n2)), "0.000")

End Sub

Public Function fishf( $f$ As Double, n1 As Double, n2 As Double)

Dim $p$ As Double

Dim v As Double, dv As Double

Dim x As Double, Y As Double, w As Double

Dim th As Double, a As Double, sth As Double, cth As Double, b As Double, b1 As Double

Dim c As Double, pi As Double, pid2 As Double, k As Double

$\mathrm{pi}=3.14159265358979$

pid2 $=$ pi $/ 2$

$\mathrm{b}=\mathrm{n} 1 / 2$

$\mathrm{b} 1=\mathrm{n} 2 / 2$

$\mathrm{x}=\mathrm{n} 2 /\left(\mathrm{n} 1{ }^{*} \mathrm{f}+\mathrm{n} 2\right)$

If $\mathrm{n} 1$ Mod $2=0$ Then

fishf $=\operatorname{statcom}(1-x, n 2, n 1+n 2-4, n 2-2) *\left(x^{\wedge}(n 2 / 2)\right)$

Exit Function

End If

If $\mathrm{n} 2 \mathrm{Mod} 2=0$ Then

fishf $=1-\operatorname{statcom}(x, n 1, n 1+n 2-4, n 1-2){ }^{*}\left((1-x)^{\wedge}(n 1 / 2)\right)$

Exit Function

End If

th $=\operatorname{Atn}(\operatorname{Sqr}(n 1 * f / n 2))$

$\mathrm{a}=$ th $/$ pid2 


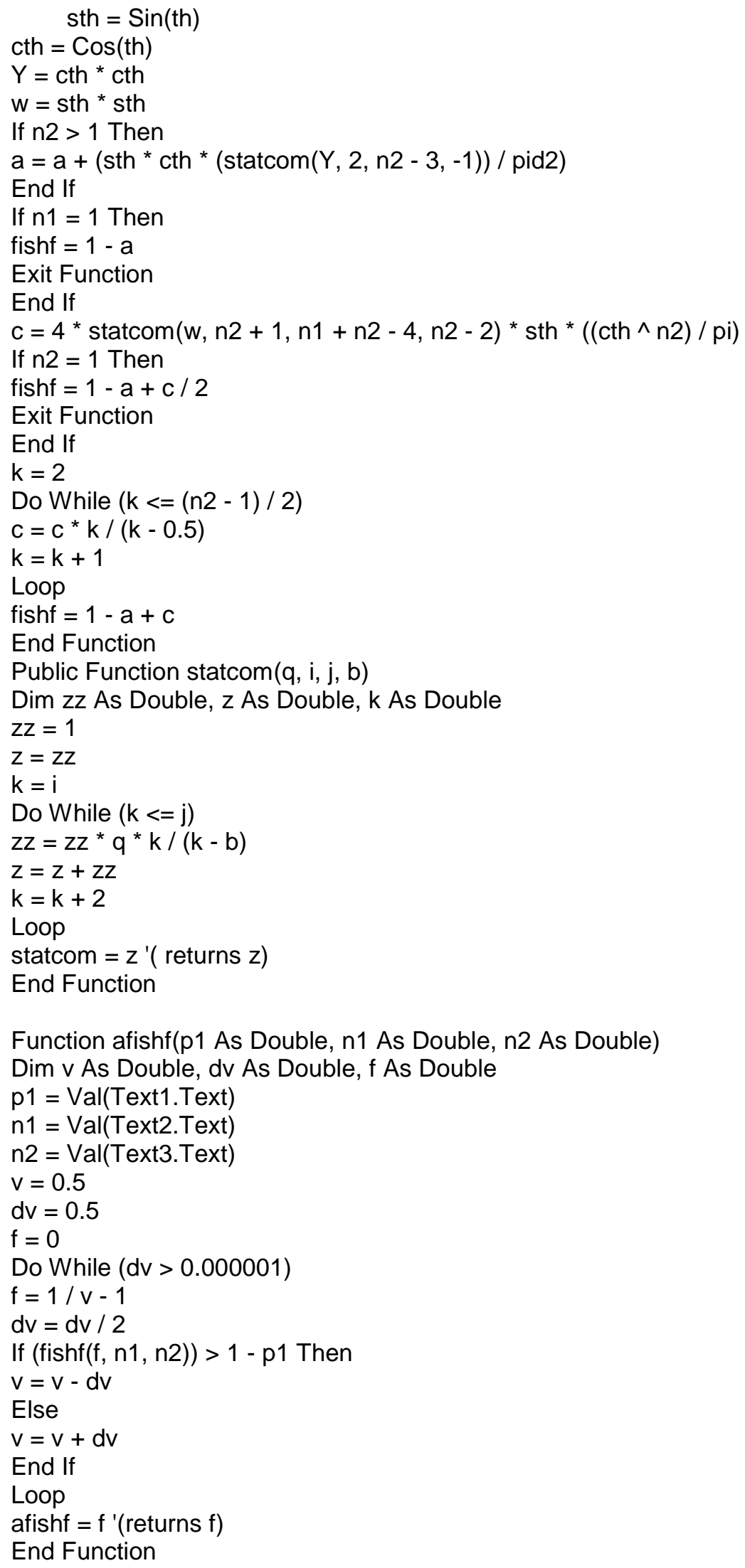

\section{$\underline{\text { Chi-square Calculator }}$}

Option Explicit 
Private Sub cmdprob_Click()

Dim cal_p As Double, p As Double

Dim n As Integer

Dim x As Double

Dim k As Double, t As Double, a As Double

Dim v As Double, dv As Double

$v=0.5$

$\mathrm{dv}=0.5$

$\mathrm{X}=0$

If IsNumeric(txtx.Text) Then

GoTo 10

Else

MsgBox "invalid number"

End If

Exit Sub

$10 \mathrm{p}=\mathrm{Val}(\mathrm{txtx}$. Text)

$\mathrm{n}=\mathrm{Val}(\mathrm{txtdf}$.Text)

Do While ( $d v>0.000001)$

$\mathrm{x}=1 / \mathrm{v}-1$

$\mathrm{dv}=\mathrm{dv} / 2$

cal_ $p=\operatorname{Exp}\left(-0.5^{*} \mathrm{x}\right)$

If $n$ Mod $2>0$ Then

cal_p $=$ cal_p * $\operatorname{Sqr}\left(2{ }^{*} \times / 3.14\right)$

End If

$\mathrm{k}=\mathrm{n}$

Do While $(\mathrm{k}>2$ Or $\mathrm{k}=2)$

cal_p $=$ cal_p * $(x / k)$

$\mathrm{k}=\mathrm{k}-2$

Loop

$\mathrm{t}=$ cal_p

$\mathrm{a}=\mathrm{n}$

Do While $\left(t>0.000001{ }^{*} p\right)$

$a=a+2$

$\mathrm{t}=\mathrm{t}^{*}(\mathrm{x} / \mathrm{a})$

cal $\_p=$ cal $\_p+t$

Loop

If $(1$ - cal_p) $>1$ - $p$ Then

$v=v-d v$

Else

$\mathrm{v}=\mathrm{v}+\mathrm{dv}$

End If

Loop

Iblp.Caption = Format $(\operatorname{Str}(\mathrm{x})$, "0.000")

End Sub

\section{Tieset Source Code $\left(\mathrm{C}_{++}\right)$}

// Manish.cpp : Defines the entry point for the console application.

\#include "stdafx.h"

\#include "Readfile.h"

\#include "Rel.h"

int main(int argc, char* argv[])

\{

if $(\operatorname{argc}==2)\{$

Rel r(argv[1]);

r.makeTree();

r.makeMat();

r.printMat();

\} 


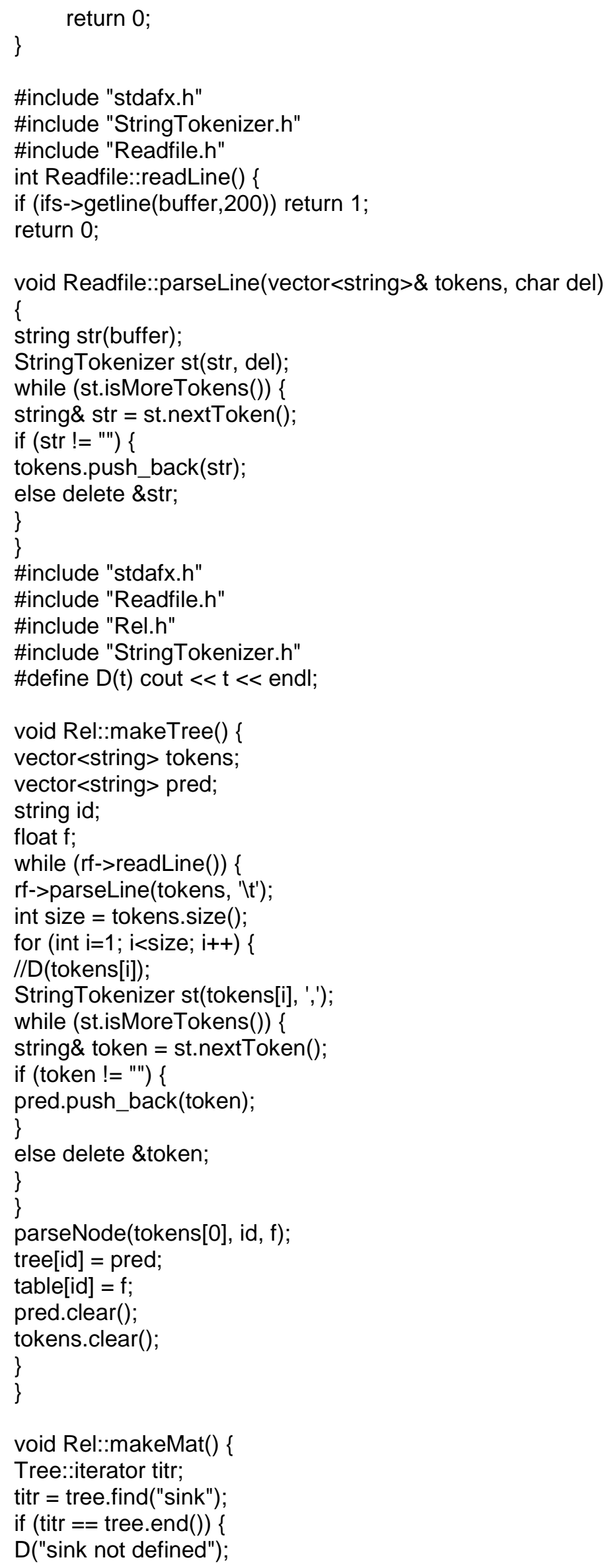




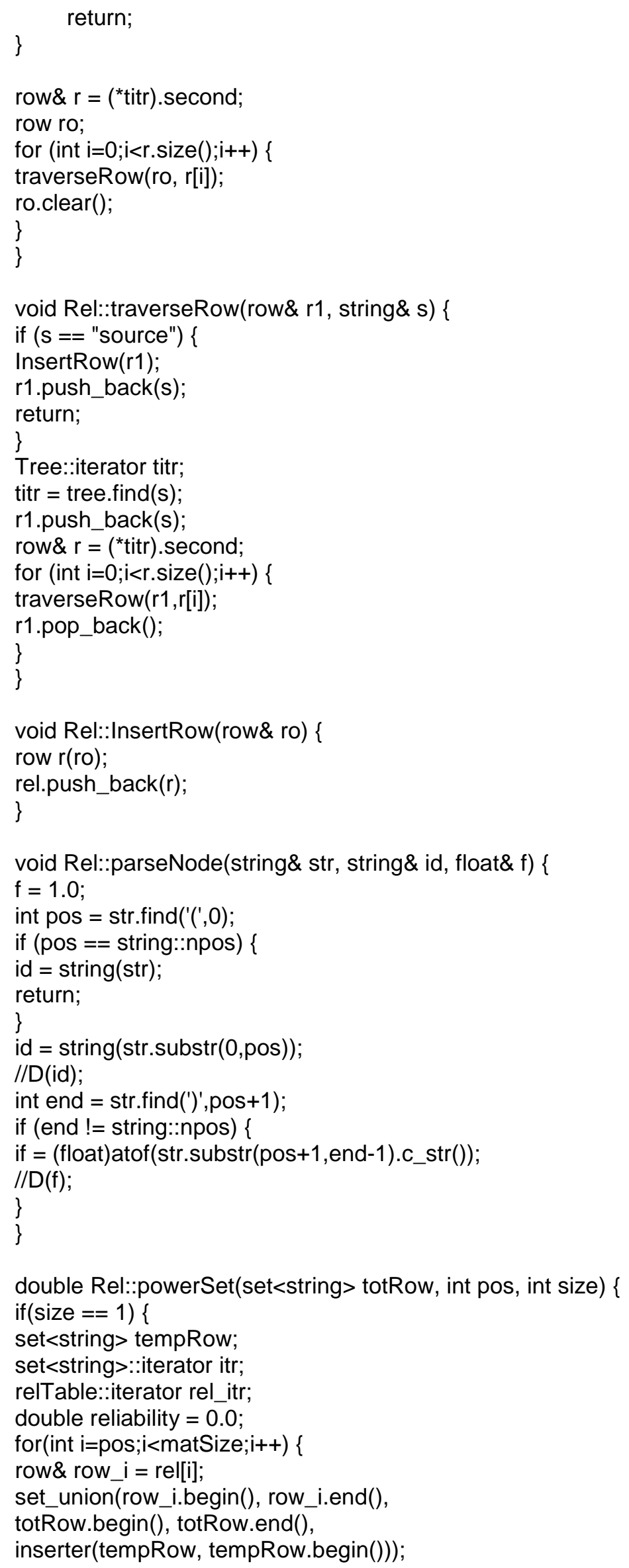




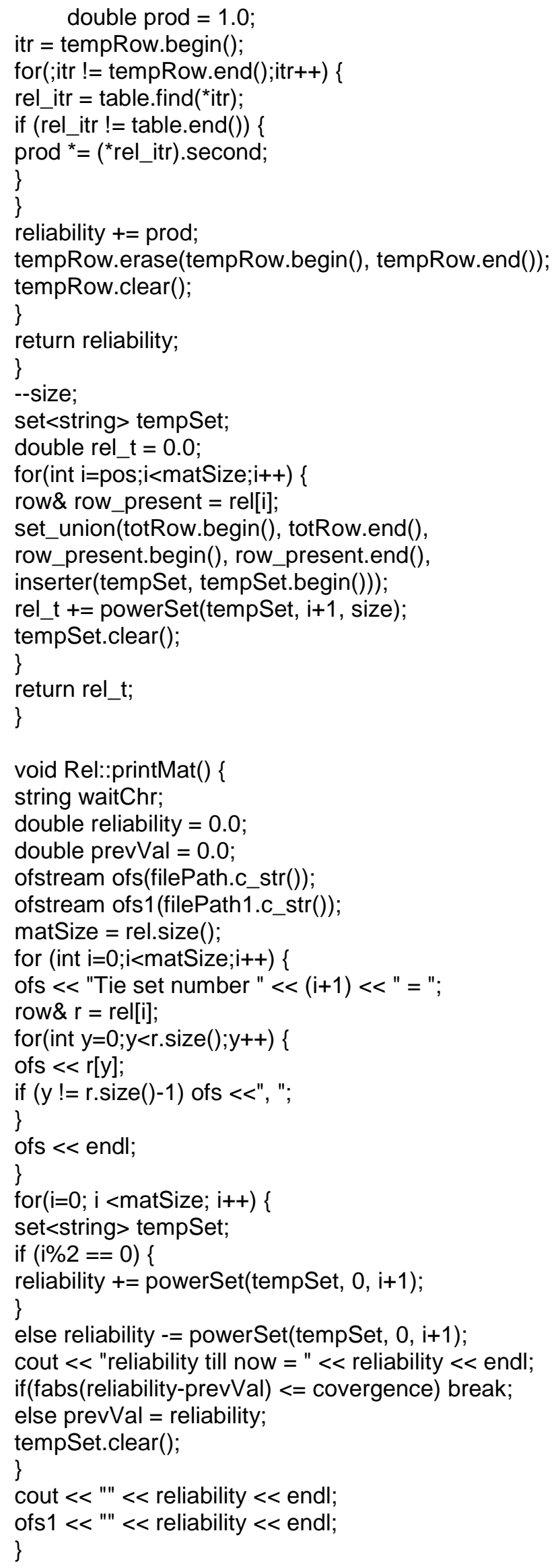




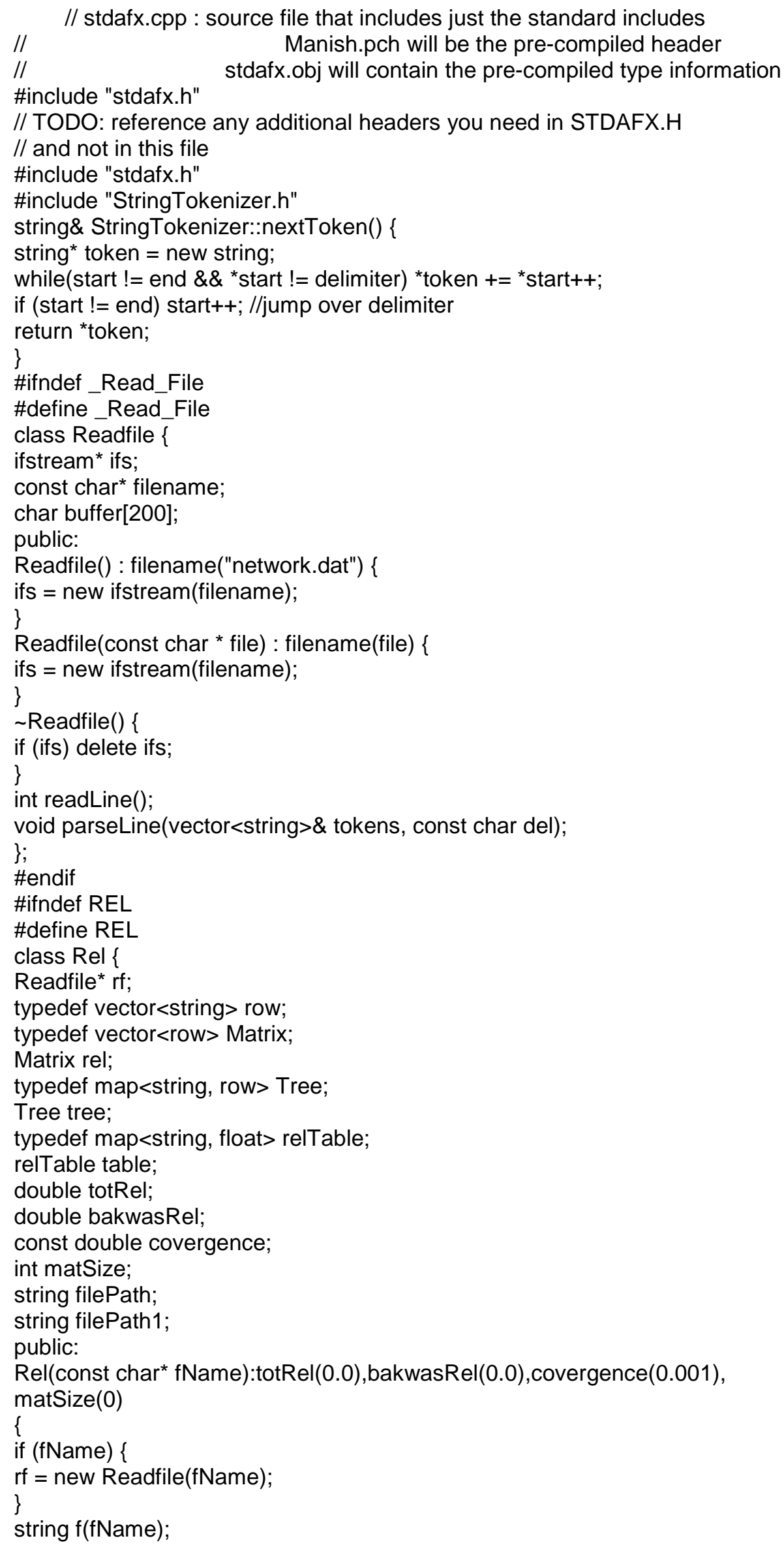




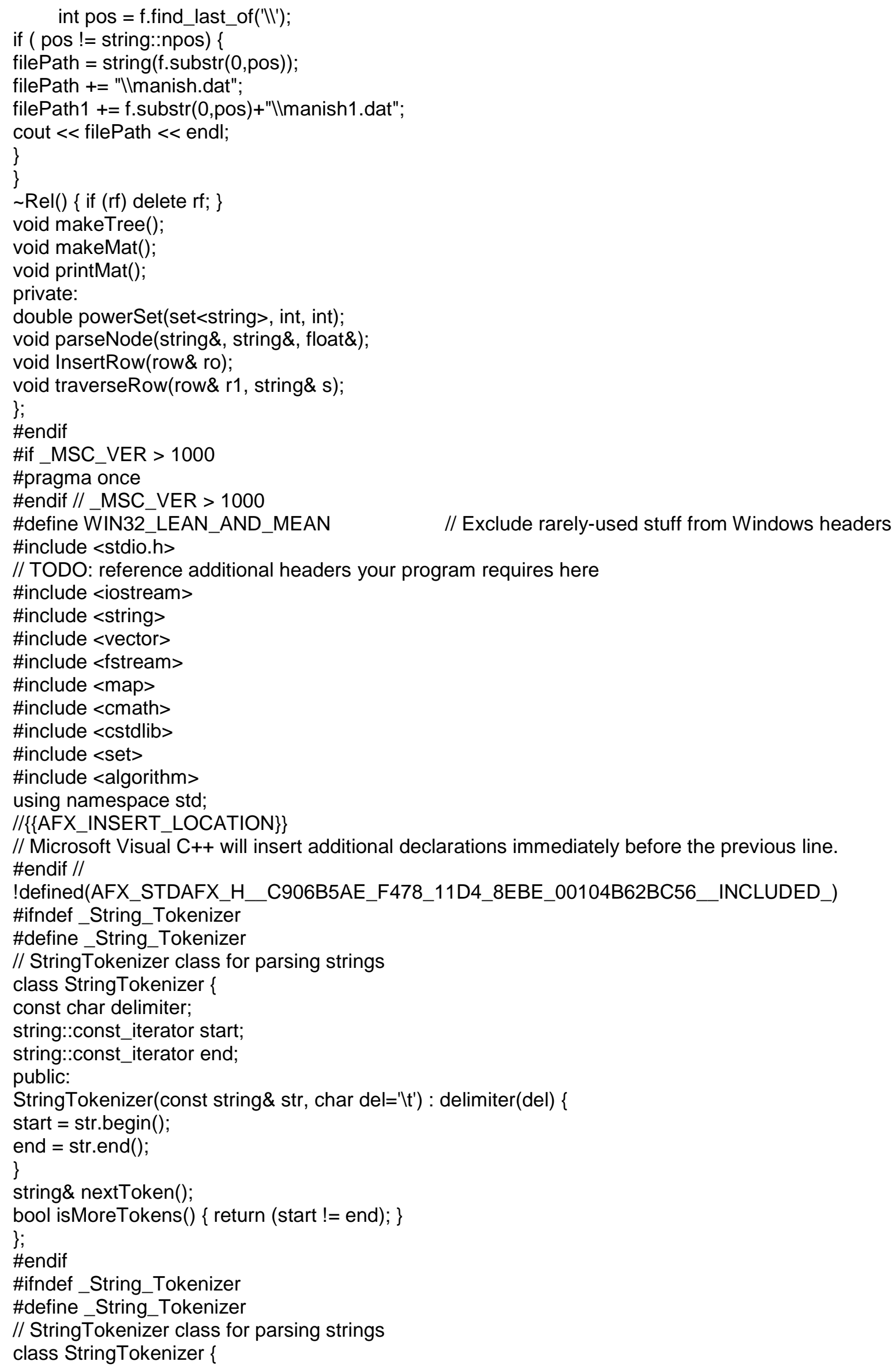


const char delimiter;

string::const_iterator start;

string::const_iterator end;

public:

StringTokenizer(const string\& str, char del='|t') : delimiter(del) \{

start $=$ str.begin () ;

end $=$ str.end () ;

\}

string\& nextToken();

bool isMoreTokens() \{ return (start != end); \}

;

\#endif 